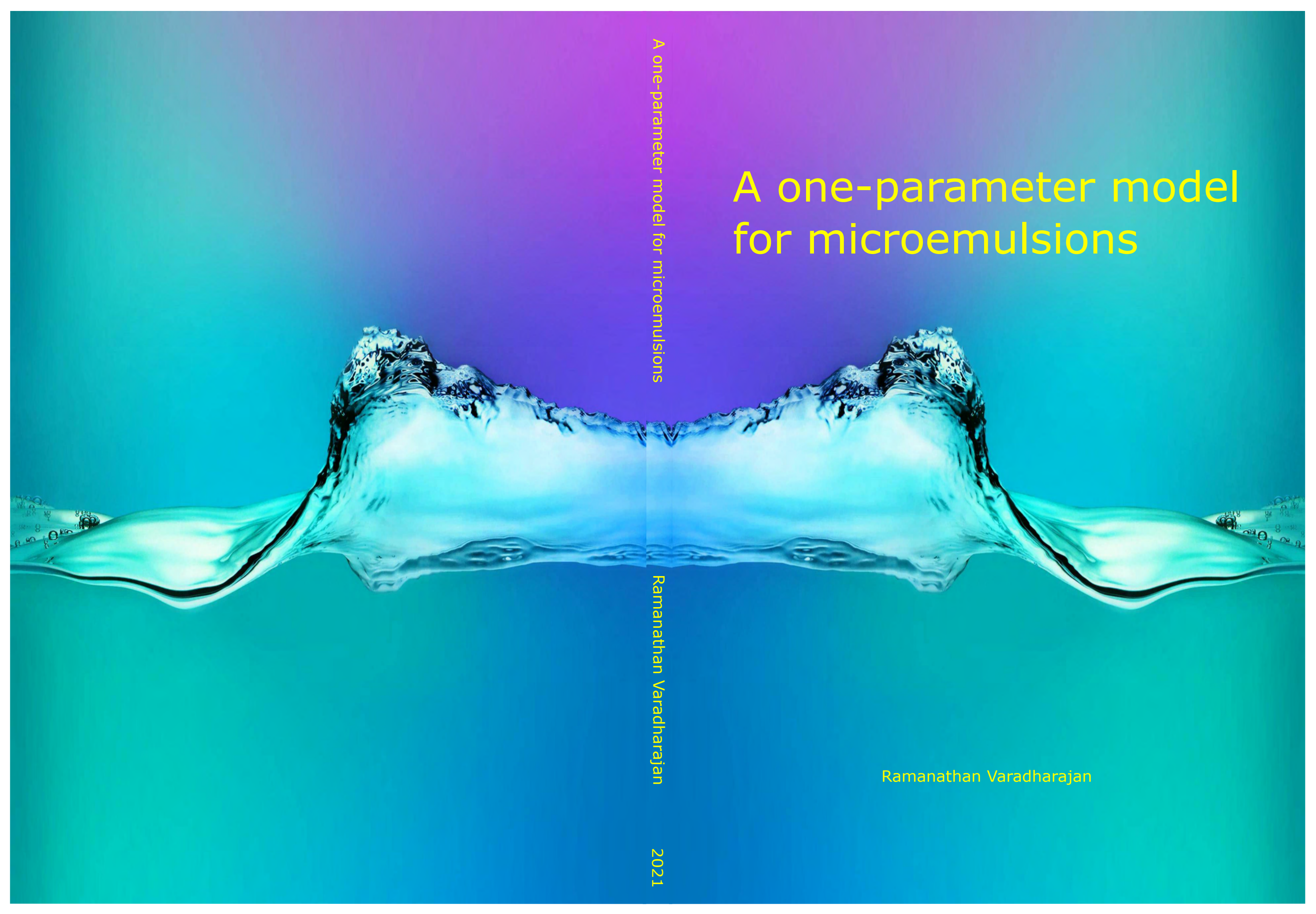




\section{Propositions}

1. Scale-invariant topologies provide unambiguous estimates of the bending rigidities.

(this thesis)

2. Critical points of a ternary system play a pivotal role in the physics of microemulsions.

(this thesis)

3. Cash-flow in a capitalistic society is analogous to a fluid flow with a negative diffusion coefficient: good governance can stabilize the former and prevent it from becoming a singularity like the latter.

4. Placing scientific knowledge behind paywalls hinders the development of human capital.

5. Badminton is aerodynamically a more advanced sport compared to tennis.

6. By imposing interpretations in works about contemporary culture, norms, and lifestyle, South-Indian films have become a frivolous criticism of the status quo.

7. Inheritance helps sustain and widen the wealth gap and increases the longevity of existing social inequalities.

8. Blitz format of chess is an excellent tool to improve the performance of an active scientific mind.

Propositions belonging to the thesis, entitled

A one-parameter model for microemulsions

Ramanathan Varadharajan

Wageningen, 12 May 2021 


\section{A one-parameter model for microemulsions}

Ramanathan Varadharajan 


\section{Thesis committee}

\section{Promotor}

Prof. Dr Frans A. M. Leermakers

Personal chair, Physical Chemistry and Soft Matter

Wageningen University \& Research

\section{Other members}

Prof. Dr A. van Blaaderen, Utrecht University

Dr E. M. Blokhuis, Leiden University

Prof. Dr J.G.E.M. Fraaije, Leiden University

Prof. Dr E. van der Linden, Wageningen University \& Research

This research was conducted under the auspices of the Graduate School VLAG

(Advanced studies in Food Technology, Agrobiotechnology, Nutrition and Health Sciences). 


\title{
A one-parameter model for microemulsions
}

\author{
Ramanathan Varadharajan
}

\section{Thesis}

submitted in fulfilment of the requirements for the degree of doctor

at Wageningen University

by the authority of the Rector Magnificus

Prof. Dr A.P.J. Mol,

in the presence of the

Thesis Committee appointed by the Academic Board

to be defended in public

on Wednesday May 12th, 2021

at 4:00 p.m. in the Aula. 
Ramanathan Varadharajan

A one-parameter model for microemulsions 148 pages.

PhD thesis, Wageningen University \& Research, Wageningen, NL (2021)

With references, with summary in English

ISBN: 978-94-6395-703-8

DOI: https://doi.org/10.18174/541239 
Educate, organize, agitate!

- Bahasaheb Dr. B. R. Ambedkar M.A., Ph.D., D.Sc., Barrister-at-Law.

Can caste be annihilated?

Not unless those who call themselves revolutionary develop a radical critique of Brahminism. Not unless those who understand Brahminism sharpen their critique on capitalism. And not unless we read Babasaheb Ambedkar. If not inside our classrooms, then outside them. Until then we will remain what he called the "sick men" and women of Hindustan, who seem to have no desire to get well.

- The Doctor and the Saint, Arundhati Roy

In solidarity with everyone who is voicing against oppression by capital, caste, gender, language, power, race, religion ...

To Appa, Amma and Dr. Subashree... 



\section{Contents}

$\begin{array}{ll}\text { Contents } & 7\end{array}$

$\begin{array}{lr}\text { List of Figures } & 9\end{array}$

$\begin{array}{ll}\text { List of Tables } & 11\end{array}$

1 Introduction 1

1.1 Brief review of microemulsions . . . . . . . . . . . . . . . 2

1.2 Applications of microemulsions . . . . . . . . . . . . . 5

1.3 Models for microemulsion . . . . . . . . . . . . . . . . . . . 11

1.4 Outline of the thesis . . . . . . . . . . . . . 13

2 Elastic Properties of Symmetric Liquid-Liquid Interfaces $\quad 15$

2.1 Introduction . . . . . . . . . . . . . . . . . . . 16

2.2 Method and Model . . . . . . . . . . . . . . . . . 17

2.3 Results . . . . . . . . . . . . . . . . . . . 19

2.4 Discussion . . . . . . . . . . . . . . . . . . . . 22

3 Liquid-Liquid-Surfactant interfaces: Route to estimate rigidities $\mathbf{3 3}$

3.1 Introduction . . . . . . . . . . . . . . . . . . . 34

3.2 Method and Model . . . . . . . . . . . . . . . . 36

3.3 Results . . . . . . . . . . . . . . . . . . . . . . . . . . . . . . . . . . .

3.4 Summary.............................. 41

4 Liquid-Liquid-Surfactant interfaces at strong and weak segre$\begin{array}{ll}\text { gation limit } & 43\end{array}$

4.1 Introduction: . . . . . . . . . . . . . . . . . . . . 44

4.2 Scheutjens-Fleer self-consistent field theory . . . . . . . . . . . . . . 49

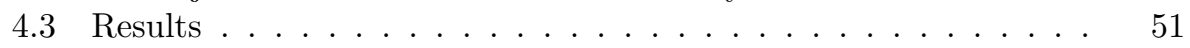


4.4 Discussion . . . . . . . . . . . . . . . . . 63

4.5 Summary .............................. 67

$5 \quad$ Imposing height undulations on the liquid-liquid interface $\quad \mathbf{7 9}$

5.1 Introduction . . . . . . . . . . . . . . . . 80

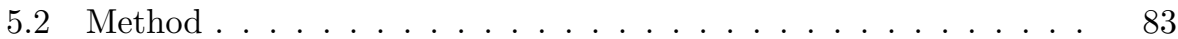

5.3 Measurables . . . . . . . . . . . . . . . . . . 89

5.4 Results . . . . . . . . . . . . . . . . . . . . 92

5.5 Discussion . . . . . . . . . . . . . . . . . . 101

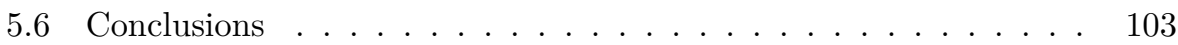

6 Summary and General Discussion $\quad 105$

6.1 Summary of our work . . . . . . . . . . . . . . . . . 106

6.2 Microemulsion modeling: Future . . . . . . . . . . . . . . . . 110

6.3 Conclusion . . . . . . . . . . . . . . . . . 116

$\begin{array}{ll}\text { Bibliography } & 117\end{array}$

$\begin{array}{ll}\text { Summary (Tamil) } & 135\end{array}$

$\begin{array}{lr}\text { List of publications } & 139\end{array}$

$\begin{array}{ll}\text { Acknowledgements } & 141\end{array}$

$\begin{array}{ll}\text { About the Author } & 143\end{array}$

$\begin{array}{ll}\text { Overview of completed training } & 145\end{array}$ 


\section{List of Figures}

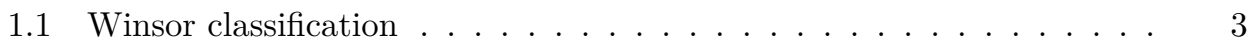

1.2 Water, decane, surfactant phase diagram . . . . . . . . . . . 4

1.3 Microemulsions in digestion . . . . . . . . . . . . . . . 6

1.4 Microemulsions in enhanced oil recovery . . . . . . . . . . . . 8

1.5 Oil recovery causing earthquake . . . . . . . . . . . . . . . . . 9

1.6 Microemulsions in pharamaceutical industry . . . . . . . . . . . . . 10

1.7 Microemulsions in advanced materials . . . . . . . . . . . . . 11

2.1 Interfacial tension and bending rigidites for Liquid/Liquid interfaces . 20

2.2 Properties of cross-over length . . . . . . . . . . . . . . . . 21

2.3 Ratio of rigidities and Diagram of states . . . . . . . . . . . . 22

2.4 Evolution of volume fraction and grand potential density profiles . . . 25

2.5 Interfacial width and rigidites close to bulk critical point . . . . . . 26

2.6 Interfacial width and rigidities - finite chain length correction . . . . . 27

2.7 Cross-over length - finite chain length correction . . . . . . . . . 28

2.8 Interfacial width and density difference close to bulk critical point . . 28

2.9 Interfacial width - finite chain length correction . . . . . . . . . . 29

2.10 Comparison of SCF results for polymeric interfaces with and without non-local interactions . . . . . . . . . . . . . . 31

3.1 Properties of planar tensionless balanced interface . . . . . . . . 36

3.2 Volume fraction distribution of Im3m cubic phase \& minimal Torus . $\quad 38$

3.3 Chain length dependence of bending rigidities . . . . . . . . . . . . 40

3.4 Bending rigidities as a function of chain length . . . . . . . . . 41

4.1 Phase diagram of a ternary system . . . . . . . . . . . . . . 45

4.2 Interfacial tension as a function of bulk surfactant concentration . . . 52

4.3 Adsorbed amount of surfactant in tensionless state . . . . . . . . . 53

4.4 Connection between Microemulsion point (MP) and Lifshitz point (LP) 55 
4.5 Volume fraction profiles for Winsor III-like system . . . . . . . . . 56

4.6 Free energy of interaction between interfaces . . . . . . . . . . . 58

4.7 Critical scaling near MP . . . . . . . . . . . . . . . . 61

4.8 Interfacial width and area expansion modulus close to MP . . . . . . . 61

4.9 Behavior of MP in response to change in surfactant size . . . . . . . 63

4.10 Illustration of interface definition . . . . . . . . . . . . . . . 69

4.11 Interfacial behavior . . . . . . . . . . . . . . . . . . . . 71

4.12 Volume fraction profiles at strong segregation . . . . . . . . . . . . . 72

4.13 Evolution of pressure profiles . . . . . . . . . . . . . . . . 72

4.14 Interactions between $\mathrm{L} / \mathrm{L}$ interfaces . . . . . . . . . . . . 76

5.1 Variation of grand potential as a function of Monte Carlo steps . . . . 93

5.2 Analysis of simulated interface . . . . . . . . . . . . . . . 94

5.3 Contours of mean, square of mean and Gaussian curvature at the traced interfacial points . . . . . . . . . . . . . . 95

5.4 Interfacial area and curvature integrals from Monte Carlo simulations $\quad 96$

5.5 Probability density function, $p(\delta h)$, of interfacial height fluctuations . $\quad 96$

$5.6 G(\delta r)$ as a function of $\delta r \ldots \ldots \ldots \ldots$. . . . . . . . . . . 97

5.7 Fourier spectrum from the interfacial height fluctuation . . . . . . . 98

5.8 Estimation of tension and bending rigidity through linear regression . $\quad 99$

5.9 Spectrum of height fluctuations of constant tension and constant mean bending rigidity simulations . . . . . . . . . . . . . . . 100

6.1 Fluctuation enhanced SCF . . . . . . . . . . . . 115 


\section{List of Tables}

5.1 Parameters of default L/L system analyzed using MC-SCF . . . . . . 92

5.2 Results of constant tension simulations . . . . . . . . . . . . . . 99

5.3 Results of constant rigidity simulations . . . . . . . . . . . . . . 100 



\section{CHAPTER 1}

\section{Introduction}

Oil and water do not mix. Oil molecules like each other and so does water molecules. Oil molecules however do not like water molecules as much as their own. Thus, there exists a effective repulsive force between oil and water molecules. Imagine pouring a glass of water into a container that has oil. Eventually, the oil will phase separate and reach the top of the container. This happens as a result of the repulsive force often referred to as the hydrophobic nature of oil. Indeed gravity dictates their position, but, interaction drives the separation. As a thought experiment, imagine oil likes water. Do you think that if we repeat the same experiment in the presence of gravity, will oil come to the top of the container? I will leave this as an open question. When oil separates from water, an interface will form between the oil-rich and water-rich regions. However, there are enormous scenarios where we would like the oil to mix completely with water. It would be extremely beneficial if we can achieve this without providing mechanical work. Such stable mixtures of oil and water can be achieved by adding a surfactant. These surfactants have both oil-loving and water-loving parts and hence assemble at the interface between oil and water. Such assembly promotes thermodynamically stable mixtures (no mechanical work required) with an enormous interfacial area. Such thermodynamically stable mixtures of oil, water and surfactant are defined as microemulsions.

To understand microemulsions. This is all this thesis is about. Our target is to generate a generic yet simplistic model to the whole class of microemulsions with accuracy at the molecular level. Firstly, we will provide a brief review of microemulsions. Later, we will present various applications of microemulsions in different fields. Finally, we will discuss existing models and conclude with an outline of the thesis. 


\subsection{Brief review of microemulsions}

As stated above, microemulsions are stable mixtures of oil, water, and surfactant. Such mixtures often show common features of ternary systems (3 component systems) and added to this also feature its own characteristics. Phase diagrams are a straight forward way to quantify the behavior. In simple terms, phase diagrams are basically a representation of what kind of phases arise when oil, water, and surfactant are mixed at a certain ratio and temperature. Before we review the known phase behavior of oil-water-surfactant mixtures, let us formally define the necessary requirement for the third component to qualify as a surfactant.

In a system consisting of oil and water, let's imagine adding a third component. Earlier we stated that this third component leads to a spontaneous mixture of oil and water. Not all molecules can qualify as a surfactant. This third component can be called as a surfactant, only if it has a capability to partition (settle) itself between the two (oil and water) phases. Non-ionic n-alkyl polyglycol ethers like $\mathrm{CH}_{3}\left(\mathrm{CH}_{2}\right)_{i-1}\left(\mathrm{OCH}_{2} \mathrm{CH}_{2}\right)_{j} \mathrm{OH}$ are an excellent candidate as surfactants. We will refer to them in the commonly accepted short notation $\mathrm{C}_{i} \mathrm{E}_{j}$. The $\mathrm{C}$ part of these surfactants prefers to stay in oil and the E part prefers to stay in water. Thus most molecules self-assemble at the interface between oil and water. Furthermore, ionic amphiphiles, such as sodium-bis-ethylexysulfosuccinate (AOT) or sodium dodecyl sulfate (SDS) can also behave as our choice for the third component. In this thesis, we will only consider the non-ionic surfactants. The reason for this is as stated before our target is to create a simplistic model. Ionic interactions, though interesting, will add complexity to our model.

In the presence of surfactant, that partitions mostly itself at the interface, we can apply an external field to control this partition behavior of the surfactant. In the case of non-ionic surfactants, the external field is temperature, and for an ionic molecule, the external field can be salt concentration. Furthermore, a fourth component called co-solvent can also be added to tune the phase behavior. It should be now clear that the choice of non-ionic surfactant is to remain generic, simple and fundamental in our work. Thus we have oil, water, non-ionic surfactant as three components and temperature as an external field.

Let's get back to our system of oil and water. For the sake of argument, let's assume we have equal amounts of oil and water. Even though we would like our surfactant to perfectly partition at the interface between oil and water, that would be an ideal scenario. Often surfactant prefers one of the two phases (oil or water). As stated before we have to tune the partition behavior using an external field like temperature. For the sake of argument, let's assume that the surfactants are mostly found in the aqueous (water) phase. In this scenario, the water phase with surfactants will be in equilibrium with the excess (pure) oil phase. Surfactants in the water will lead to the formation of droplets of oil inside the water phase. 
1.1. Brief review of microemulsions

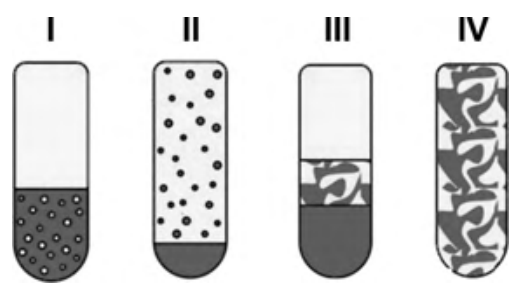

Figure 1.1: Winsor classification of microemulsion. Illustration of different phases formed when oil, water and surfactants are mixed. Winsor Type I - represents an oil phase coexisting with droplets of oil in water. Winsor type II - represents an water phase coexisting with droplets of water in oil. Winsor type III system has an excess oil and excess water phases coexisting with a middle phase. Middle phase grows and engulfs the excess oil and water phases to form Winsor IV - single phase microemulsion. Reprinted from [1] with permission.

Winsor classified such microemulsions as Winsor I (oil-in-water microemulsions), see Fig 1.1. By increasing the temperature of the system a middle phase with more of surfactant and less of oil and water will appear. The middle phase will thus have an intermediate density between that of an oil-rich and water-rich phase. This is classified as the Winsor III system. The temperature at which the middle phase appears is defined as the lower critical temperature $\left(\mathrm{T}_{L}\right)$. On increasing the temperature further, the surfactants will become increasingly soluble in oil compared to that of water. Again we will end up forming a two-phase system with pure water phase in equilibrium with droplets of water in oil. The temperature at which this happens is called the upper critical temperature $\left(\mathrm{T}_{U}\right)$ and the system is classified as Winsor II. So far we have increased the external parameter (Temperature) at a fixed oil, water, surfactant ratio. However, a second parameter that can be used to study the system property is the concentration of the components. To do so, and yet remain simple, let's look at the balanced systems wherein the amount of oil is equal to the amount of water. For this 1:1 (oil: water) system at a certain temperature, we can increase the surfactant concentration. At a certain concentration, a single-phase appears in which the excess oil and excess water are solubilized. Such systems are called as Winsor IV systems. In this thesis we often refer to this system as microemulsion.

Let's take a look at an example system and observe how different phases feature in reality. In Fig. 1.7, Phase diagrams for non-ionic amphiphile, oil, water ternary systems are presented. It can be seen that in all of these phase diagrams, a fish-like structure exists. Within the body of the fish, a three-phase region (Winsor III) is observed. The Head of the fish marks the beginning of the three-phase region in the phase diagram. The body of the fish ends in a tail where the single-phase 

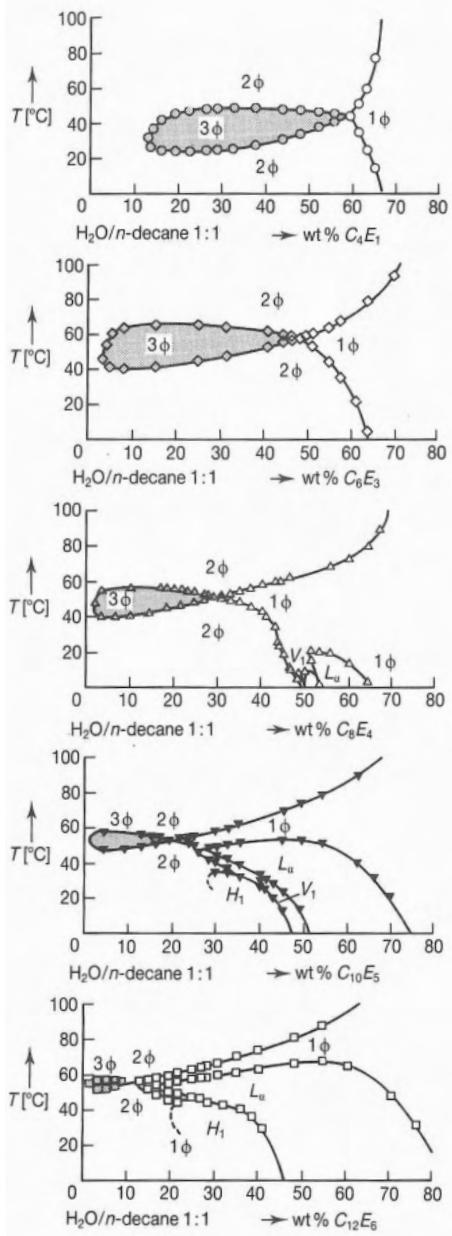

Figure 1.2: Phase diagram of water, decane and $\mathrm{C}_{i} \mathrm{E}_{j}$ surfactant. Ratio of water to oil (decane) is 1:1. Surfactant strength is varied from top to bottom by increasing the number of units of $\mathrm{C}$ and $\mathrm{E}$. Temperature of the system $\left[\mathrm{in}{ }^{\circ} \mathrm{C}\right]$ and surfactant concentration [in wt\%] are used as two control parameters. Two phase regions are denoted by $2 \phi$, Winsor III is represented as $3 \phi$. Winsor IV is represented as $1 \phi$. Lamellar $\left(\mathrm{L}_{\alpha}\right)$, Hexagonal $\left(\mathrm{H}_{1}\right)$ and $\mathrm{V}_{1}$ phases are also seen at certain conditions. Reproduced with permission from [2] 
region (Winsor IV) occurs. Above and below the head and body of the fish, Winsor $\mathrm{I}$ and Winsor II (two-phase regions) are observed. This is clearly seen for the surfactants $\mathrm{C}_{8} \mathrm{E}_{4}-\mathrm{C}_{4} \mathrm{E}_{1}$. For larger surfactants, the phases in the tail region are more complicated with lamellar, hexagonal and cubic phases.

It can be seen as the chain length of the surfactant is increased from $\mathrm{C}_{4} \mathrm{E}_{1}$ to $\mathrm{C}_{12} \mathrm{E}_{6}$ the body of the fish becomes smaller. Further, the head of the fish and the tail of the fish move to the left of the phase diagram with lower surfactant concentration. This means that a single-phase region is observed at a lower surfactant concentration for a longer surfactant. Thus a surfactant strength can be weakened by decreasing both the number of carbons in the hydrophobic tail and by decreasing the number of ethoxy groups in the surfactant head. However, using a strong surfactant doesn't necessarily mean that the single-phase region will be bicontinuous (disordered mixtures). It can be seen from the phase diagram for the $\mathrm{C}_{12} \mathrm{E}_{6}$, the single-phase region is more likely to be in a hexagonal or lamellar regime with a very narrow regime of bicontinuous phase marked as $1 \phi$. This shows that knowledge about the interfacial properties and the structure of the observed single-phase region is essential to explain more about the behavior of microemulsions.

Another important thing to note is the temperature. It can be seen as the surfactant chain length is increased (or strength is increased), the head of the fish moves to a higher temperature. Even though it is not very clear for the tail of the fish, a similar trend can be observed from the figure.

Models and experiments attempt to understand this behavior. Experiments are a great tool to observe the phase behavior of specific surfactants. However, as always, a generic computer model will not only help design the right experiments but also provides a fundamental understanding of the observed phase behavior. Indeed, many models and years of expertise have shed a broad understanding of microemulsions, yet there remains a huge gap. Our knowledge of microemulsions remains to be a drop in an ocean of unknowns. Before we explain more about the existing models, let's explore the necessity to know more about microemulsions. Indeed, the necessity arises from enormous potential applications of these mixtures.

\subsection{Applications of microemulsions}

Even though discovery and technical definition of microemulsion happened in 1957 [4], the last two decades saw applications of microemulsion in various fields such as oil recovery, pharmaceutical industries, fuels, lubricants, corrosion inhibitors, cosmetics, reaction kinetics, liquid membranes, food technology and so on. It is interesting to note, definitely not recommended, that the oil was thrown into seawater to calm the waves in the early days [5] and remains to be the first application of oil-water interactions. 


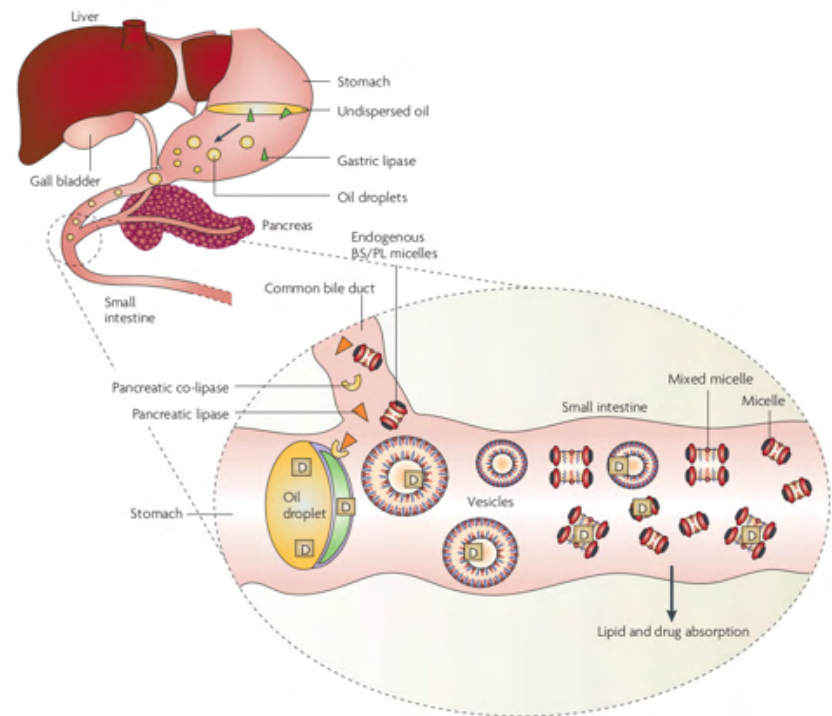

Figure 1.3: During ingestion, lipid, triglycerides and the drug forumlation are taken orally. Following ingestion, digestion is initiated in the stomach by gastric lipase. Primary function of the gastric lipase and associated pancreatic lipase and co-lipase was arguably to breakdown the ingested oil (triglycerides) into a series of colloidial structures. These colloidial structures include multilamellar/unilamellar vesicles, micelles, mixed micelles etc., Indeed, these colloidial structures can be closely related in a fundamental level to the oil-in-water droplets in Winsor I systems. Reprinted from [3] with permission.

\section{Food Industry}

Let's start with some delicious applications of microemulsions. Milk, flavored milk products, creams, whipped cream, butter, cheese, salad dressings, mayonnaise, coffee whitener, soups, yogurt, ice cream, etc., are common examples of food emulsions. Let's note that food emulsions are not necessarily microemulsions. The primary difference between food emulsions and microemulsions is that food emulsions are not thermodynamically stable. This comes with a drawback. Food emulsions (commonly macroemulsions) require kinetic energy to be stabilized. This is often achieved in the food industry through a process called homogenization. Importantly, the stability, size of these food emulsions dictate the shelf-life, texture, taste, etc., Wouldn't it be nice to have food products that have shelf-life comparable to that of honey? This can potentially be achieved with thermodynamically 
stable food microemulsions. Considering microemulsions are more stable than the (macro)emulsions, it is tempting to promote micro emulsification in the food technological process. There is a catch. Most food products have an oil phase made of triglycerides. Triglycerides are weak compared to hydrocarbon oils and cannot form an oil-rich phase. Furthermore, food regulations place a huge constraint on the range of surfactants that can be used. Food grade surfactants such as phosphatidylcholine (lecithin), AOT and sorbitan monostearate/monolaurate (Tweens) are few of the most studied, leaving a huge range of surfactants unexplored. Also, it is not a wonder that microemulsions naturally occur in a range of foods. It is speculated that microemulsion phases form during the digestion and absorption of fat and lipophilic drugs, see Fig. 1.3.

Currently, the primary application of microemulsions within the food industry is to use microemulsions as a carrier for bioactive food compounds [6-9]. This regime of research has interdisciplinary applications. We will revisit this when we discuss the applications in the pharmaceutical industry. The necessity to obtain more insights into the formation of food-grade microemulsions is clear. In our thesis, by attempting to model microemulsions in a generic context we hope to have provided the food microemulsion community with additional insights. However, it might be stated that understanding the micelle formation (droplets of lipids) can help also understand the process of human digestion [10-12].

\section{Oil and gas industry}

A novel approach to enhance oil recovery uses microemulsions. Energy needs are rising, while supply is staggering. High energy needs directly influence the climate and environment. To understand how microemulsions can play a key role in the oil industry let's take a brief look at oil recovery processes. As shown in Fig. 1.1, a typical oil field consists of an injection well and a production well. These wells run several thousand meters deep into the surface of the earth and reach the oil-reservoir beds. Oil-reservoir beds are basically sediments of fossils over billions of years, turned into a mixture of crude oil trapped between porous rocks. Through the injection wells, a huge quantity of water is pumped in to recover these trapped crude oil through the production well. Typically a huge amount of saltwater is recovered along with the crude oil. This wastewater has to be disposed of. The amount of wastewater produced is directly related to the efficiency of the oil recovery process. Before we discuss how microemulsions can improve the efficiency of oil recovery and drastically reduce wastewater production, let's look at how this wastewater is disposed of in the current industrial set up.

Though the wastewater is sometimes recycled to use for hydraulic fracturing, most industries inject the wastewater deep into the earth using wastewater disposal wells as shown in Fig. 1.2. Such a process is known to have several implications on 

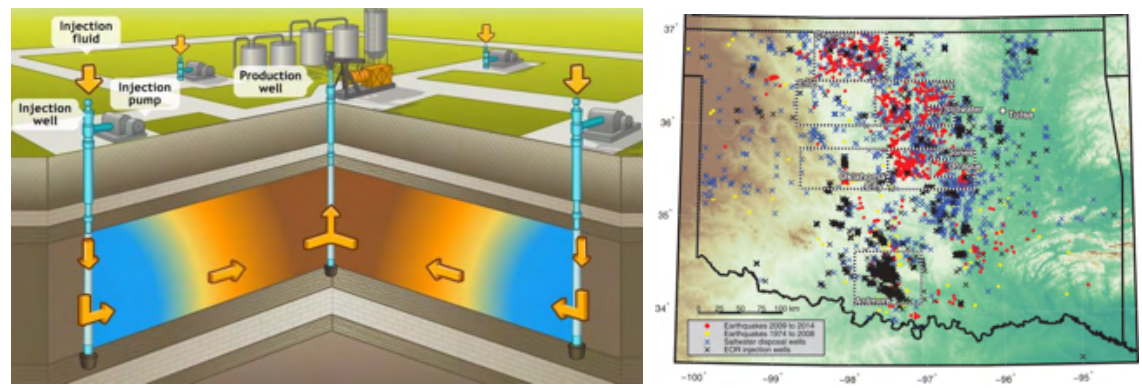

Figure 1.4: Microemulsions in enhanced oil recovery. Figure in the left illustrates a typical oil field process (Source: Reprinted with permission from SNF Floerger). Primary components of these processes include an injection well, production well, and storage tanks. Water and surfactants are pumped through the injection well. Injected water washes out the trapped oil and pumps out through the production well because of pressure difference. On the right a study correlating locations of wastewater pumps with earthquakes in the city of Oklahama is presented. Black crosses represents injection wells, blue crosses represent saltwater disposal wells and the red dots represent the earthquakes recorded during the years between 2009 2014. Reprinted from [13] with permission.

the frequency and magnitude of earthquakes [13]. In a recent study, earthquakes were directly correlated to the oil excavation process in the city of Oklahoma, US (see Fig. 1.3). Furthermore, as operations expand the volume of wastewater generated is reported to increase exponentially [14, 15]. Environmental impacts of the produced wastewater are enormous and being increasingly realized by both the scientific and industrial community [16-18]. Considering the growing demand for energy, unless an alternative scalable energy source is found, oil and natural gas are going to be the primary source of energy. Thus there will be expansions in operations and an increase in environmental impacts. As said before, by increasing the efficiency of the tertiary oil recovery, a significant amount of wastewater produced can be reduced. The efficiency of the tertiary oil recovery process is directly linked to the ability of the drive water (water pumped through an injection well) to mix with the trapped oil. Here is where the application of microemulsions comes into the picture. Typically, in oil recovery, a huge amount of surfactant will be pumped in to achieve microemulsion phases in the reservoir bed. These processes aim to reach Winsor IV phase in a (crude oil, drive water, surfactant system). By gaining insights into the fundamental behavior of oil, water, surfactant system can thus help mitigate the environmental degradation caused due to low-efficiency oil exploration techniques. 


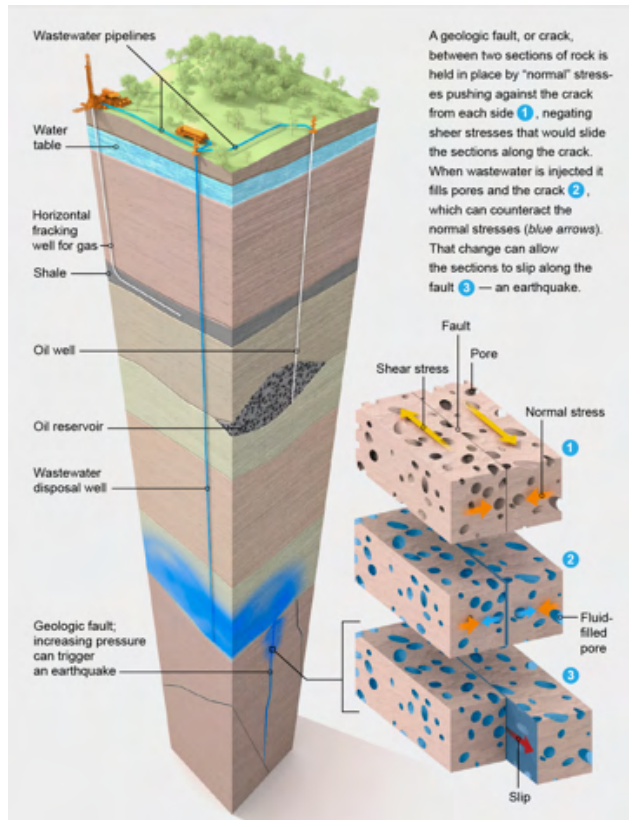

Figure 1.5: Oil recovery processes as causation of earthquakes. Illustration of observed correlation presented in previous figure. As stated in the figure, geological cracks are held in place by normal forces. Pumping wastewater fills up the crack and has a possibility to counteract the normal forces. Inherently, a slip becomes more probable in fluid filled porous rocks. Furthermore, pumped saltwater is reported to affect the quality of watertable in several regions. Source: PBS Newshour

\section{Pharmaceutical industry}

A drug often consists of an active ingredient and an excipient. Activie ingredient is the primary component required to address a specific symptom and a drug can have one or more active ingredients. An excipient is the component of the drug used to either tune its long term stability, adsorption, viscosity etc., Microemulsions act as an excellent drug carrier and lie within the category of excipient carrying the active ingredient in the drugs. One example is provided in the Fig. 1.4, where lipid based micelles are used to carry the active ingredients to ensure target based drug-delivery on cancer cells. In this study [19], authors suggest a way to target cancer cells while leaving the normal cells. This is achieved using nanoparticles. Understanding micelle stabilization (in this case the nanoparticle stability) is directly linked to the microemulsion systems with drugs (replacing the oil phase) and blood (replacing 


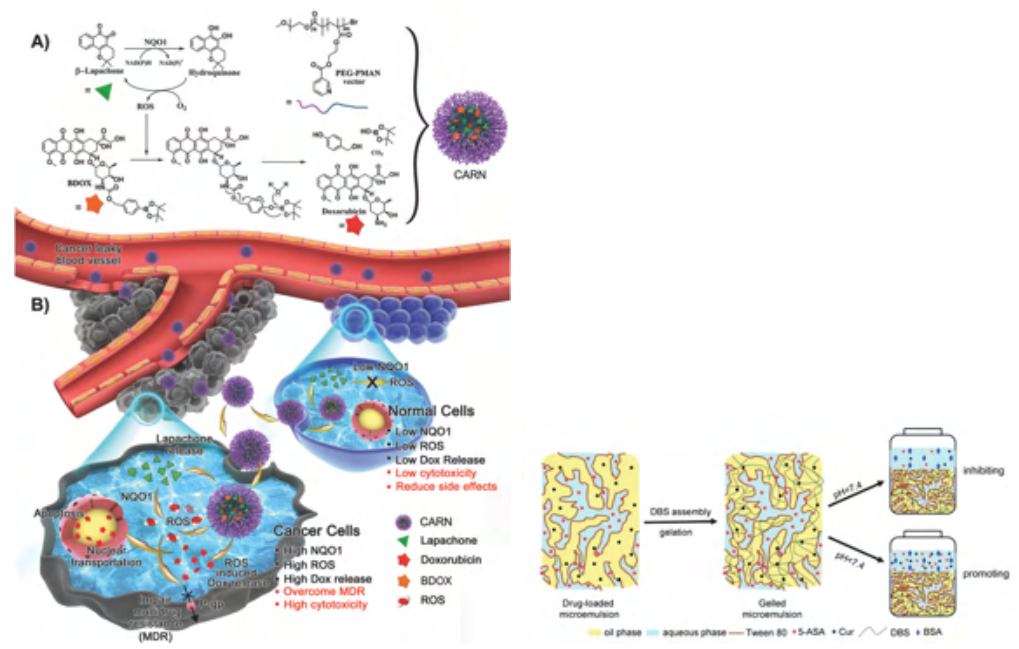

Figure 1.6: Microemulsions in pharamaceutical industry. On the left, A cascade amplification release nanoparticle (CARN) is constructed by the coencapsulation of $\beta$-lapachone and a reactive-oxygen-species (ROS)-responsive doxorubicin (DOX) prodrug, BDOX, in polymeric nanoparticles. Structural stability of CARN is related to their Winsor I, II phase-behavior in a medium of blood or intracellular fluids. Reprinted from [19] with permission. On the right, microemulsions were prepared by using Tween 80 as a surfactant, 1,2-propylene glycol as a co-surfactant, isopropyl myristate as an oil phase and phosphate-buffered saline as an aqueous phase. The resultant microemulsions possess a bicontinuous-phase structure. The microemulsions were gelled by the self-assembly of sorbitol derivatives as a gelator to form novel supramolecularly gelled microemulsions that can be used as the carrier of a dual-drug, such as hydrophilic 5-aminosalicylic acid and lipophilic curcumin. Reprinted from [20] with permission.

the water phase) and lipids (replacing the surfactant).

\section{Advanced materials}

Advanced materials is a developing field in the branch of material science. From transistors to anti-microbial fabrics variety of essential technological applications has been a direct result of the research in the field of advanced materials. It is however not straightforward to imagine the role of microemulsions in such a field. However, microemulsion play a significant role in the field of advanced materials. We will show a few example. In a study published in 2017 [21], a new 
formulation based on water in oil in water $(\mathrm{w} / \mathrm{o} / \mathrm{w})$ microemulsions made from a plant extract was used as coating on cotton fabric. UV cured coated fabric showed an excellent anti-microbial property. Such materials not only improve the standard of living but also provide a means to contain the spread of pandemics. In Fig. 1. 4, bicontinuous interfacially jammed emulsion gel (bigel) fibre development is presented. Microemulsion formulation plays a key role in this process [22-24]. Such microfibres have potential applications in medicine. Bijels with food grade surfactant can lead to more stable products with longer shelf-life as discussed above.

Overall, a sound fundamental knowledge on microemulsions (ternary systems) provides with quality food, cleaner energy, efficient drugs and high performance materials, to say the least. A plethora of applications remain unexplored because of our limited knowledge on microemulsions.
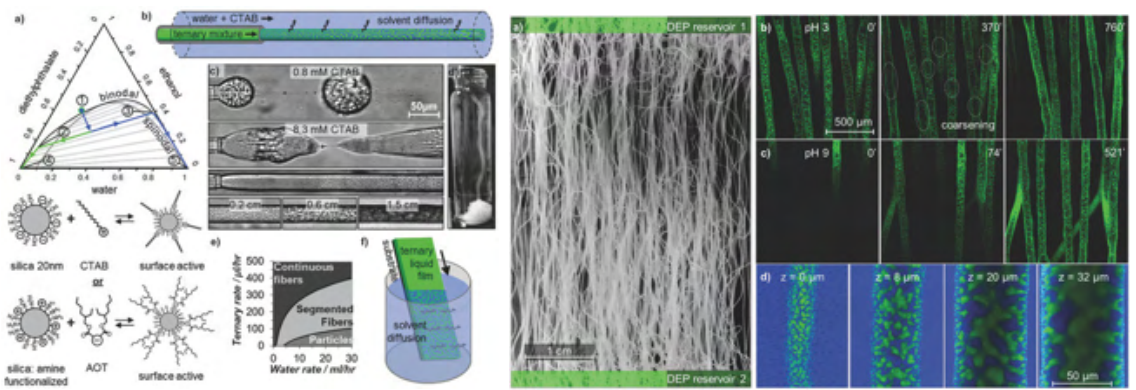

Figure 1.7: Microemulsions in advanced materials. Continuous generation of hierarchical and asymmetric bijels based on solvent-transfer-induced phase separation (STRIPS) is demonstrated. In STRIPS, phase separation is induced by solvent extraction from an initially homogeneous ternary mixture, and bicontinuous morphology is stabilized by interfacial attachment of nanoparticles, which are functionalized in situ. STRIPS allows stable bijel formation from a wide variety of liquids and particles. On the right, visualizations of bijel fibres are presented. Reprinted from [22] with permission.

\subsection{Models for microemulsion}

Any model describing a physical phenomena can be classified based on its resolution (range of length scales captured). Microemulsions are amazingly complex to model as the range of length scale required to capture the whole behaviour ranges from a few nanometres to several hundreds of micrometres. We will outlay a few models that exist at different length scales. 


\section{Atomic models}

All-atom molecular dynamics simulations that can resolve details of individual atoms can be classified as the atomic models [25]. The atomic-level resolution provides detailed information about the structure and dynamics of the surfactants at the interface between oil and water. Note, often the dynamics can be observed not beyond several nanoseconds. This inherent limitation in the total simulations time arises from the stringent stability criterion arising from the velocity Verlet algorithm. To break it down, let's say that advancing the simulation from time $t$ to $t+1$ is only physically accurate as long as the $\Delta t=(t+1)-t$ lies within a certain range. This range is directly linked to the spatial resolution of the simulations. Since the atomic models aim at increasing the spatial resolution, the drawback is to compromise in the time evolution. Atomic models are a great tool to understand the in-depth molecular behaviour at short time scales and short length scales (typically few hundred nanometers). Beyond that it is computationally intensive and mostly impossible even with present-day capability of GPUs and parallelization to achieve a mesoscopic outcome from atomic models.

\section{Microscopic models}

The second class of models, take the basic entities of the model to be the molecular components of the system, and the fundamental length scales observed are at molecular scales. In microscopic models, all components are replaced by their statistical equivalents with no internal structure. Thus interactions are modelled by a few parameters or functions and are inherently short range. Often such interactions are made attractive between polar entities (water and a head group of surfactant) and non-polar entities (oil and tail group of surfactant). A repulsive interaction is present between polar and non-polar entities (water and oil). Scheutjens-Fleer self-consistent field theory (SF-SCF) falls within this category and so does other mean-field theories.

Most of these models require computers to achieve a solution and thus require a discrete space replacing the continuum theory. It is however argued that meanfield theoretical approaches are accurate based on the assumption that lattice discretisation errors does not influence the problem. It is easy to see that the earlier assumption is valid in the limit of weak gradients (in systems where the gradients in properties take more than a few discrete layers to change). In strong segregations (steep change of properties), such discretisation often lead to lattice artefacts.

With extensively fast computational time, microscopic models are often employed to understand the phase behaviour [26-29], microemulsion structure [30-32] and interfacial and wetting properties of ternary systems. This shows the power and applicability of mean-field theories towards understanding microemulsions. In this thesis, we will employ SF-SCF to understand microemulsions. 


\section{Membrane models}

We have discussed atomic-level models and molecular level models earlier. Imagine modelling an entire sheet of surfactants assembled at the interface between oil and water. Basic entity of these models is thus the behaviour of the sheets given by their rigidity. The sheets of assembled surfactants can be monolayers (in oil, water, surfactant systems) or bilayers (in water, surfactant systems). We will focus on monolayers that are applicable for microemulsions. Monolayers are considered as incompressible two-dimensional liquids with a characteristic area per head group in membrane models.

It is easy to see that such membrane models are the easiest way to study the fluctuations of single monolayers. This can be directly quantified by studying the energy required to bend these monolayers, called as mean bending rigidity $(\kappa)$. Monte-Carlo simulations of such monolayers is straight forward.

The problem arises when we attempt to study the single-phase microemulsions. The disordered Winsor IV systems have multiple monolayers and thus require statistical mechanics of random surfaces. It should be noted, that Monte Carlo simulations of more than one sheet has never been performed yet. In this thesis, we will show that the fluctuation enhanced self-consistent field theory is the way forward to understand Winsor IV systems.

\subsection{Outline of the thesis}

In this thesis, we explore the potential of a one-parameter molecular model to describe the behaviour of microemulsions. Oil, water and surfactant are modeled as $\mathrm{A}_{n}, \mathrm{~B}_{n}, \mathrm{~A}_{N} \mathrm{~B}_{N}$, where $\mathrm{n}, \mathrm{N}$ represent chain lengths. One-parameter, $\chi$ is used to dictate repulsion between $\mathrm{A}$ and $\mathrm{B}$. This parameter combines the effect of intermolecular interactions and external field (Temperature). Thesis is divided into two parts. In part 1, We employ Scheutjens Fleer self-consistent field theory (SF-SCF) to understand the equilibrium properties of the system. In part 2, we develop and present a hybrid Monte-Carlo SCF simulation approach to include the effects of interfacial fluctuations in SF-SCF to understand the off-equilibrium properties. Finally, we provide a overview of Fluctuation-enhanced self-consistent field theory (FL-SCF). Below a chapter-wise overview is provided.

In chapter 2 we establish an unambiguous route to study the properties of symmetric liquid-liquid interface (oil-water interface). In this work, we do not consider the addition of a third component, and thus the interfaces always feature a finite interfacial tension $\gamma$. We report a positive value for both moduli when the system is close to critical and the rigidities show the same scaling as $\gamma$. Furthermore, we find that the length scale $\lambda$ remains of the order of the segment size. 
In chapter 3 we add a ternary component (surfactant) to the binary system. Since the surfactant adsorbs spontaneously to the liquid-liquid interface, tension drops. We explore the tensionless state in such systems where the bending moduli dictates the physics of the interfaces. Such interfaces are called as saturated interfaces. Focus is to establish an elegant protocol to determine the rigidities of saturated interfaces. We explore scale-invariant geometries like a minimal Willmore torus and $\operatorname{Im} 3 \mathrm{~m}$ cubic phases and report that $\bar{\kappa}$ switches sign upon approaching the weak segregation limit.

In chapter 4 we analyze the ternary system discussed in chapter 3 in both strong and weak segregation limit, by varying the interaction parameter $\chi$ and the surfactant concentration $\varphi_{s}^{b}$. We reproduce a phase diagram in $\chi-\varphi_{s}^{b}$ and add regions relevant for microemulsions. Specifically, we report that the saturation line (line of tensionless states) ends at a critical point. We refer to this as the Microemulsion point (MP). Furthermore, we show that in the limit of infinitely long polymers MP merges to form the classical Lifshitz point. Within the phase diagram, we show a pair of binodal envelop the three-phase region with a middle phase. While analyzing the interaction between the saturated interfaces, a non-monotonic interaction was observed reasoning the cohesiveness between the interfaces in the observed middle phase.

In chapter 5 we present the implementation and theory behind the hybrid Monte-Carlo self-consistent field theory approach to study off-equilibrium interfacial fluctuations. We demonstrate this using the binary system discussed in chapter 2 . Using the fluctuation route bending rigidities are obtained and compared with the mean-field results. Potential applications of the developed method are discussed.

In the final chapter, we present the feasibility of fluctuation enhanced selfconsistent field theory to include the bulk-like fluctuations in the system. Though we present some preliminary results towards such an approach we show that there are complexities that are needed to be resolved. We leave this as an open question for future research. We conclude by discussing our results and provide a summary. 


\section{CHAPTER 2}

\section{Elastic Properties of Symmetric Liquid-Liquid Interfaces}

The mean $(\kappa)$ and Gaussian $(\bar{\kappa})$ bending rigidities of liquid-liquid interfaces, of importance for shape fluctuations and topology of interfaces, respectively, are not yet established: even their signs are debated. Using the Scheutjens Fleer variant of the self-consistent field theory, we implemented a model for a symmetric L/L interface and obtained high precision (mean field) results in the grand canonical $(\mu, V, T)$-ensemble. We report positive values for both moduli when the system is close to critical where the rigidities show the same scaling behavior as the interfacial tension $\gamma$. At strong segregation, when the interfacial width becomes of the order of the segment size, $\bar{\kappa}$ turns negative. The length scale $\lambda \equiv \sqrt{\kappa / \gamma}$ specifies the cross-over from the interfacial tension dominated- to the bending rigidity dominated damping of interfacial fluctuations, remains of order the segment size for all strengths of interaction; yet the $1 / \sqrt{N}$ chain length correction reduces $\lambda$ significantly when the chain length $N$ is small. 


\section{$2.1 \quad$ Introduction}

Thermal energy makes liquid-liquid (L/L) interfaces to undergo undulations and wavelike fluctuations. On a large length scale, beyond the capillary length, these are damped by gravity. On mesoscopic length scale it is the surface tension $\gamma$, which counteracts the area change that accompanies these fluctuations. On the nanoscale the bending of the interfaces are damped by the interfacial bending rigidity $\kappa$. More specifically, out-of-plane fluctuations of $\mathrm{L} / \mathrm{L}$ interfaces are controlled by the interfacial tension for wavelengths larger than the cross-over length $\lambda=\sqrt{\kappa / \gamma}$ and by the bending rigidity $(\kappa)$ at shorter wavelengths. This $\lambda$ should be comparable to a molecular length scale [33]. Precise prediction of $\lambda$ from a molecular model would significantly advance our understanding on fluctuations in L/L interfaces. However, molecular models that have access to $\kappa$ at sufficient accuracy have not yet been forwarded. A key issue here is that molecular theories thus far have failed to establish the sign of $\kappa$. This uncertainty arguably had negative impact on the recognition of $\lambda$ as a key interfacial quantity. As $\kappa$ controls the magnitude of the fluctuations (at short length scales), we expect it to be positive $(\kappa>0)$. In stark contrast to this, surprisingly few theoretical predictions foresee a positive value [34]: to date, molecular models typically predict negative values [35-38]. Nevertheless in (mesoscale) simulations [39-41] and in phenomenological models [42] a positive sign for $\kappa$ is often chosen.

Besides $\kappa$, interfaces have a second elastic constant known as the saddle spay modulus or Gaussian bending rigidity $\bar{\kappa}$. The $\bar{\kappa}$ should control the topology of interfaces; a negative value will prevent the formation of saddle shaped interfaces whereas a positive value will promote these. A sign-change (e.g. upon a change of temperature) is easily envisioned. However, existing predictions indicate a strictly positive value [35-37].

Molecular theories give relatively easy access to the accurate values for the interfacial tension $[43,44]$. However, the evaluation of the rigidities has many intricacies. With respect to common practise, we found that a sound estimation of $\kappa$ and $\bar{\kappa}$ from a molecular theory require to overcome two hurdles: (i) to quantify the curvature expenses at a fixed chemical potential $(\mu)$ of all molecular species and (ii) to properly account for non-local contributions to the enthalpic interactions.

In this chapter, we successfully overcome these theoretical challenges and show that $\kappa$ is strictly positive for $\mathrm{L} / \mathrm{L}$ interfaces and hence fluctuations from planar state cost free-energy. We observe that $\lambda$ is of the order of the segment size in the limit of strong or weak segregation, yet shows a non-monotonous behavior in transition regime. We discuss the implications of chain length $(N$ - degree of polymerization) on fluctuations of L/L interfaces, in light of results obtained for $\lambda$. Finally, we present and discuss the sign-switch for $\bar{\kappa}$. 


\subsection{Method and Model}

Mean field results for a simple symmetric interface between two liquids $A_{N}$ and $B_{N}$, where $N$ is chain length (degree of polymerization), is discussed. The case with $N=1$ correspond to the well-known van der Waals interface [44, 45]. When $N$ is large we arrive at another well studied interface, namely between two inmiscible polymers [35, 46]. As $\gamma>0$, the system has a tendency to minimize its area. Thermal energy causes the macroscopic interface to fluctuate. The accompanied entropy gain is counteracted by an unfavourable increase in area and a penalty for the interface to (locally) bend away from the planar ground state. Such curved interfaces cannot maintain their tension exactly. Following Helfrich [47] we consider a Taylor series expansion of the tension in terms of the mean $\left(J=1 / R_{1}+1 / R_{2}\right)$ and Gaussian $\left(K=1 / R_{1} \times 1 / R_{2}\right)$ curvature $\left(R_{1}\right.$ and $R_{2}$ are two principle radii of curvature):

$$
\gamma(J, K)=\gamma(0,0)+\frac{\partial \gamma}{\partial J} J+\frac{1}{2} \frac{\partial^{2} \gamma}{\partial J^{2}} J^{2}+\frac{\partial \gamma}{\partial K} K+\cdots
$$

The term linear in $J$ is well documented and properly understood [48-52]. Here and below, we will focus on symmetric interfaces for which this linear term vanishes. Defining the mean bending modulus, $\kappa \equiv \frac{\partial^{2} \gamma}{\partial J^{2}}$, and Gaussian bending rigidity, $\bar{\kappa} \equiv$ $\frac{\partial \gamma}{\partial K}$, Eqn. 2.1 reduces for weakly curved interface to $\gamma(J, K)-\gamma(0,0)=\frac{1}{2} \kappa J^{2}+\bar{\kappa} K$. The grand potential, $\Omega=\int \gamma d A$, quantifies the excess free energy of the interface and is the characteristic function that can be used to describe the fluctuations of the interface that take place at specified chemical potentials, $\mu$, (that of the binodal) of the molecular species in the system.

\section{Scheutjen-Fleer Self Consistent Field Theory}

We implement a self-consistent field model using lattice approximations as introduced by Scheutjens and Fleer for polymer adsorption [53, 54]. These authors combined a freely jointed chain model with a Flory-Huggins equation of state. The repulsive interactions between $A$ and $B$ segments is quantified by a Flory-Huggins interaction parameter. When $\chi>\chi^{c}=2 / N$ the system features a solubility gap. It turns out that it is important to understand how the SF-SCF formalism deviates from the classical SCF theory that is used to describe microphase segregation (which is also frequently used to model the interface between two polymeric solutions).

In SF-SCF we write a mean field free energy (in dimensionless units) for a molecularly inhomogeneous system [55-58] in terms of volume fraction $\varphi_{x}(r)$ and complementary segment potential $u_{x}(r)$ profiles for segment types $x=A, B$ on a grid of lattice sites with characteristic size $b$ equal to segment size. To facilitate proper extremization we add a Lagrange parameters, $\alpha(r)$, in free energy to implement the local incompressibility constraint, $\varphi_{A}(r)+\varphi_{B}(r)=1$, applicable in 
each coordinate $r$ and a parameter $\Delta_{r ; r_{0}} \equiv \delta_{r ; r_{0}} \nu$, where $\delta_{r ; r_{0}}$ is unity when $r=r_{0}$ and zero otherwise and $\nu$ is the Lagrange parameter coupled to the requirement that at the interface location $r=r_{0}$ the density of both components match [35]:

$$
\begin{gathered}
F=-\ln Q([u])-\sum_{x, r} u_{x}(r) \varphi_{x}(r) L(r)+F^{i n t}([\varphi])+ \\
\sum_{r} \alpha(r)\left[\sum_{x} \varphi_{x}(r)-1\right]+\Delta_{r ; r_{0}}\left[\varphi_{A}(r)-\varphi_{B}(r)\right]
\end{gathered}
$$

In the mean field approach one can decompose the partition function $Q=\Pi_{i} q_{i}^{n_{i}} / n_{i}$ ! where $i=1,2$ refers to $A_{N}$ and $B_{N}$ respectively. The molecular partition function $q_{i}$ contains the statistical weight of all possible and allowed conformations of molecule $i$ (see below). $n_{i}$ is the number of molecules of type $i$ in the system. $L(r)$ gives the number of lattice sites at the lattice coordinate $r$. For planar system $L(r)=1$ is a constant (all quantities are per unit area), in cylindrical coordinates $L(r) \propto r$ (and quantities are expressed per unit length of the cylinder), while in spherical coordinates $L(r) \propto r^{2}$. The interaction free energy is a function of the densities:

$$
F^{i n t}=\chi \sum_{r} L(r) \varphi_{A}(r)\left[\left\langle\varphi_{B}(r)\right\rangle-\varphi_{B}^{b}\right]
$$

Here $\varphi_{B}^{b}$ refers to the bulk volume fraction of B (of one of the bulk phases). Importantly, the angular brackets are needed to account for the contact energy in a system with gradients in density. Similar as in the Cahn Hilliard theory [45] we write

$$
\left\langle\varphi_{B}(r)\right\rangle=\varphi_{B}(r)+\frac{1}{6} \nabla^{2} \varphi_{B}(r)
$$

where the $\nabla^{2}$ is easily mapped on the lattice as explained extensively in earlier literature $[53,55]$. SCF solutions now involve optimizing the free energy $(F)$ with respect to its variables, respectively segment potentials, volume fractions. When $\partial F / \partial \varphi_{A}(r)=0$, we find that the potentials must obey $u_{A}(r)=\alpha(r)+\Delta_{r ; r_{0}}+$ $\chi\left(\left\langle\varphi_{B}(r)\right\rangle-\varphi_{B}^{b}\right)$ (and similarly for $\left.u_{B}(r)\right)$. Setting $\partial F / \partial u_{x}(r)=0$ shows the way to evaluate the densities: $\varphi_{x}(r)=-\partial \ln Q / \partial u_{x}(r)$. The molecular partition functions are found from the endpoint distribution $q_{i}=\sum_{r} G_{i}(r, N \mid 1)$. The endpoint distributions are recursively found from $G_{i}(r)=\exp -u_{i}(r)$ by the propagator $G_{i}(r, s \mid 1)=G_{i}(r)\left\langle G_{i}(r, s-1 \mid 1)\right\rangle$, where the angular brackets have the same meaning as in Eqn.2.4. The segment densities are found by the composition law, which for homopolymers read: $\varphi_{i}(r)=\sum_{s} C_{i} G_{i}(r, s \mid 1) G_{i}(r, N-s-1 \mid 1) / G_{i}(r)$. As the position of the interface is already controlled by a Lagrange parameter $\Delta_{r ; r_{0}}$, we no longer need to specify a fixed amount of one of the components (as is needed in a canonical ensemble), but we can normalize the densities with $C_{i}=\varphi_{i}^{b} / N_{i}$ where 
$\varphi_{i}^{b}$ is specified by the binodal: A proper binodal value is a (relevant) root of the Flory-Huggins Eqn. $\ln \frac{\varphi}{1-\varphi}+\chi N(1-2 \varphi)=0$.

Numerical solutions, which routinely were obtained with an accuracy of 9 significant digits, that optimize the free energy functional and obey to all constraints, have the property that the potentials both determine and follow from the volume fractions profiles and vice versa and are said to be self-consistent. For such solution one can compute the grand potential by $\Omega=\sum_{r} L(r) \omega(r)$ wherein the grand potential density at coordinate $r$ is given by $\omega(r)=-\sum_{i}\left(\varphi_{i}(r)-\varphi_{i}^{b}\right) / N_{i}-$ $\alpha(r)-\chi\left(\varphi_{A}(r)\left[\varphi_{B}(r)+\frac{1}{6} \nabla^{2} \varphi_{B}(r)\right]-\varphi_{A}^{b} \varphi_{B}^{b}\right)$. The planar interface has a tension $\gamma_{p}=\sum_{z} \omega(z)$, where $z$ is the coordinate in the planar system. This planar interface serves as the ground state or reference state needed to estimate the grand potential increase of the curved interfaces.

\subsection{Results}

SCF solutions are routinely created for planer (p) cylindrical (c) and spherical (s) coordinates. As the position of the interface is exactly known and specified by $\Delta_{r ; r_{0}}$ to be at coordinate $r=r_{0}$ we obtain the interfacial tensions in all cases unambiguously. In spherical geometry we have $R \equiv R_{1}=R_{2}=r_{0}-1 / 2$, while in cylindrical geometry $R \equiv R_{1}=r_{0}-1 / 2$ and $R_{2}=\infty$ and $\gamma_{s}=\Omega_{s} /\left(4 \pi R^{2}\right)$ and $\gamma_{c}=\Omega_{c} /(2 \pi R)$ for spherical and cylindrical geometries, respectively. Here $\Omega_{s}$ is the grand potential when the interface is curved in a spherical fashion, and $\Omega_{c}$ is the grand potential per unit length of the interface when curved in a cylindrical fashion. Here we have implemented that a lattice site at coordinate $r$ is a distance $r-1 / 2$ away from the center of the coordinate system. In all calculations we make sure that the numerical value for $r_{0}$ significantly exceeds the width of the interface. Next we compute $\gamma_{c}-\gamma_{p}$ as well as $\gamma_{s}-\gamma_{p}$ that use the Helfrich equation 2.1 to extract with high accuracy both $\kappa$ (from cylindrical geometry) and $2 \kappa+\bar{\kappa}$ (from spherical geometry). The combination of these results leads to both $\kappa$ and $\bar{\kappa}$. Note that in all calculations, $\mu$ for the molecular species were set to the value at the appropriate binodal. Invariably, we find a positive value for the mean bending modulus whereas the sign of $\bar{\kappa}$ is (as expected) not fixed.

Results for $N=1$ (the van der Waals interface[43, 44]) are presented in SI, here we focus on the captivating results for $N>1$ and understand that $N=1$ is a limiting case.

In Fig. 1(a), we present the interfacial tension and in Fig. 1(b) the bending rigidities, both as a function of $\Delta \chi$, where $\Delta \chi \equiv \chi-\chi^{c}\left(\chi^{c}=2 / N\right.$ : bulk critical point), for three values of the chain lengths $N=2,20$ and 200. The corresponding results for the density difference and the interfacial width are presented [59]. The results for the interfacial tension are in principle well known [35, 45, 46, 60-62]. As long as the interface is wide compared to the coil size we find $\gamma \propto N(\Delta \chi)^{3 / 2}$ and 


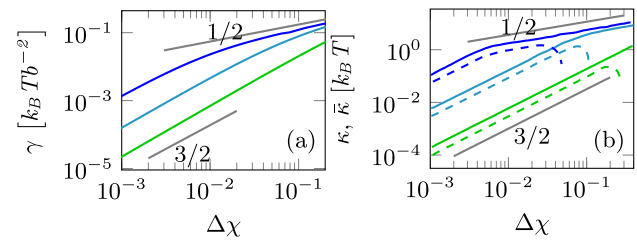

Figure 2.1: (a) Interfacial tension per unit area $\gamma$ in units of $\left[k_{B} T b^{-2}\right]$ as a function of $\Delta \chi=\chi-\chi^{c}$ in log-log coordinates (b) Mean $\kappa$ (solid line) and Gaussian bending rigidities $\bar{\kappa}$ (dashed line) in units of $\left[k_{B} T\right]$ as a function of $\Delta \chi$. Relevant slopes are indicated. Three different chain lengths were used $(N=2:$ green, $N=20$ : cyan, $N=200$ : blue).

in the other limit, where the interfacial width is small compared to the coil size, we have $\gamma \propto(\Delta \chi)^{1 / 2}$. (The $3 / 2$ exponent is the mean field prediction, known to be subject to changes, the $1 / 2$ exponent is expected to be accurate.) As in this regime $\chi$ exceeds $\chi^{c}$ a lot, the result is similar to the known result that $\gamma \propto \sqrt{\chi}$. The latter result/regime is referred to as the strong segregation limit and the former regime will be referred to as the weak segregation limit.

Interestingly the results for both bending rigidities [cf. Fig. 1(b)] follow the results for the interfacial tension qualitatively. In the weak segregation the $3 / 2$ scaling is found, while for the strong segregation the $1 / 2$ scaling is recovered. It is important to mention that the Gaussian bending modulus deviates from the latter power-law dependence rather abruptly: quite suddenly the Gaussian bending rigidity goes to zero and then changes its sign. We will discuss this behaviour below in more detail. Comparing Figs. 1(a) and 1(b) shows that in the transition regions between weak and strong segregation the tension and rigidities do not exactly copy their dependencies. This has interesting implications as we will show next.

Above we introduced the length scale $\lambda=\sqrt{\kappa / \gamma}$. Combining results form Fig. 1(a), 1(b), we present $\lambda$ as a function of $\Delta \chi$ in semi-logarithmic coordinates for a few systems that differ in $N$ in Fig. 2(a). In this figure it can be seen that $\lambda$ goes through a local maximum $\left(\lambda^{\max }\right)$ in-between weak and strong segregation. Further, $\lambda$ reaches some fixed value, which is approximately $16 \%$ larger at weak-, compared to strong segregation. In Fig. 2(b), linear dependence of $\lambda^{\max }$ is presented as a function of chain length $N$. Computer simulations that are aimed to find the bending rigidity from the height fluctuations of the interfaces [39, 63] may benefit from relatively large $\lambda$-values and preferably should be executed for large chains, because $\lambda^{\max }$ grows linearly with $N$ as $\lambda^{\max }=0.02 N+6.54$. In passing we should note that $\kappa$ has been computed in the weak curvature limit. To reach interface fluctuations dominated by bending we need small wavelengths, and then the weak curvature approximation possibly may not suffice. Such problems may 


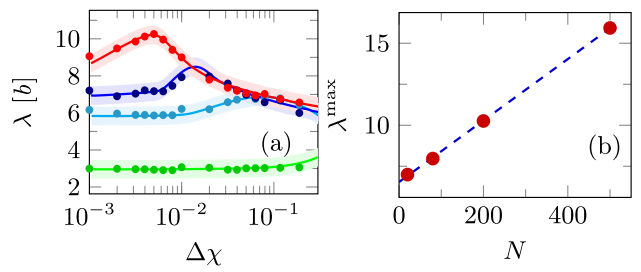

Figure 2.2: $\lambda$ as a function of $\Delta \chi$ for different $N=2$ (green), 20 (light blue), 80 (dark blue), 200 (red). Guide lines for $0.1 \%$ uncertainty in $\kappa$ are provided to visualize the trend, points show original data. (b) $\lambda^{\max }$ vs $N$ showing a linear dependence of the maximum cross-over length with respect to the chain length for $(N>20)$. Dots indicate extracted values, blue dashed line is a linear fit $\lambda^{\max }=0.02 N+6.54$.

come to the forefront when computer simulations are performed on the fluctuation characteristics of $\mathrm{L} / \mathrm{L}$ interfaces. That is why such simulations remain of more than average interest.

It is of interest to consider the ratio $\bar{\kappa} / \kappa$. Obviously, when $\bar{\kappa}$ is switching its sign, we cannot have a fixed ratio between the rigidities, but slightly outside the transition regions, that is both at weak and at strong segregation, the ratio is remarkably constant. This is illustrated in Fig. 3(a) where for a selected number of values for $\Delta \chi$-values this ratio is plotted as a function of the chain length $N$. For low values of $N$ and low values of $\Delta \chi$ we have the typical weak segregation result. It could have been concluded from Fig. 1(b), the ratio goes to a constant of approximately $1 / 2$. In the high $N$ high $\Delta \chi$ value limit, that is, the strong segregation limit, where the Gaussian bending rigidity is negative, the ratio is approximately $-3 / 2$. Implicit in this prediction is that also in the regime where $\bar{\kappa}<0$, the (negative) Gaussian bending rigidity follows a scaling law: $-\bar{\kappa} \propto(\Delta \chi)^{1 / 2}$.

Recently, we have shown for microemulsion systems that the Gaussian bending rigidity is positive for systems near the (bulk) critical point and negative otherwise [64]. From Figs. 1(b) and 3(a) it can be seen that for liquid/liquid interfaces the same phenomenology applies and it is of interest to quantify this sign switch. It is mentioned in SI [59] that the scaling for the interfacial width $W \propto(\Delta \chi)^{-1 / 2}$ applies both at weak and strong segregation regimes. The appropriate prefactor depends on $1+1 / N$ in weak segregation and on $1+1 / \sqrt{N}$ in strong segregation regime [59, 65]. In Fig. 3(b) we present a diagram of states in coordinates $\sqrt{\Delta \chi}$ vs $1 / \sqrt{N}$. The dashed line $\left((\Delta \chi)^{1 / 2}=0.2+0.5 / \sqrt{N}\right)$ separates the parameter combination with positive $\bar{\kappa}$ values from the negative ones. The line in Fig. 3(b) is functionally consistent with the prefactor for the interfacial width scaling in the strong segregation limit and therefore we speculate that the interfacial width controls the sign switch. Apparently, the Gaussian bending rigidity changes sign 
when the width of the interface is approximately 3 to 4 times the segment size. This thus happens at relatively weak segregation for short chains but in the strong segregation regime for long chains.
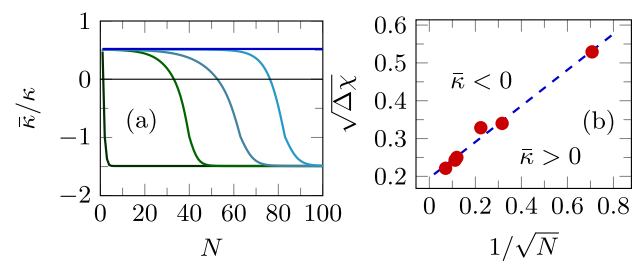

Figure 2.3: (a) Ratio of Mean and Gaussian bending rigidities as a function of molecular weight $N$ at $\Delta \chi=0.03$ (dark blue), 0.06 (light blue), 0.075 (cyan), 0.08 (green), 0.3 (black) showing transition from $\bar{\kappa} / \kappa=1 / 2 \rightarrow-3 / 2$ as the system is going from weak to strong segregation by increasing the chain length. (b) Diagram of states for the sign switch of $\bar{\kappa}$. The dashed fit-line is explained in the text.

\subsection{Discussion}

Physically, the implication of a positive $\kappa$ is that fluctuations of the $\mathrm{L} / \mathrm{L}$ interface away from the planar state do cost (free)energy. In the light of existing literature, this expected result is remarkable for several reasons.

(i) The way the interface is pinned, using a Lagrange parameter coupled to the equal density of the two liquid component, was first used by Matsen [35]. He implemented this method to find the bending rigidities using the classical self-consistent field machinery. His SCF approach has many similarities with the (current) SF-SCF approach. Yet he reported strictly negative values for $\kappa$ and positive values for $\bar{\kappa}$. The only relevant difference between our SF-SCF approach and the classical SCF approach of Matsen rests in the fact that for the interactions we have implemented the Cahn-Hilliard gradient terms, and Matsen did not. In the SI we show that when in SF-SCF these gradient terms are neglected, that is, when we implement (cf Eqn 2.4) $\langle\varphi(r)\rangle \rightarrow \varphi(r)$, we do reproduce all results of Matsen to a high accuracy. This proves the importance of the non-local interaction contributions to determine the rigidities. In the absence of these non-local interactions, neither the sign nor the scaling dependencies are apparently properly predicted.

(ii) A number of years ago Blokhuis has shown that a big effect on how bending of the interface is implemented [66] must be expected. He identified the so-called equilibrium bending mode where $\mu$ controls the curvature. In this case, the bending of the interface is accompanied by the development of a Laplace pressure inside the 'droplet' phase. $\gamma$ is then typically computed at the so-called surface of tension 
(SOT). The position of the interface is taken to be at the SOT, even though other choices can be implemented. When the tension evaluated at this SOT is used in the Helfrich equation one can evaluate $\kappa$ and $\bar{\kappa}$ (again using the combination of cylindrical and spherical geometries). As confirmed by SF-SCF calculations [37, 67], in this case $\kappa$ is negative and $\bar{\kappa}$ is strictly positive. Also for equilibrium bending one finds scaling behaviour for both moduli when the system is close to the bulk critical point. However in this case the coefficient of $1 / 2$ is found. Blokhuis also analyzed the bending of the interface at fixed $\mu$ (binodal values) by controlling the position of the interface by some (local) external field. Interestingly, in this case he recovered the $3 / 2$ scaling law, similarly as presented above for the weak segregation [68]. However, still the value of $\kappa$ was negative. Interestingly, quantitative values for $\kappa$ did depend on the choice that was implemented to define the interface position. Blokhuis could not exclude that there might be some choices for this that could turn $\kappa$ positive. Hence the current results that shows positive values for $\kappa$ and a $3 / 2$ scaling coefficient (in the weak segregation limit) indeed is the anticipated result.

Perhaps the more interesting prediction is the sign switch of $\bar{\kappa}$. In surfactant systems such sign switch has been found earlier [64] and is expected because it correlates with the rich phase behavior for these systems that include cubic phases and sponge phases. For the liquid/liquid interface the sign switch of $\bar{\kappa}$ is unknown. It will be of more than average interest to find experiments for which this sign switch is important. This is not trivial because we know that the prime interest of an interface is to reduce its area under the influence of a finite tension. However, when the interfaces are strongly perturbed, one might find that drops may pinch off. Arguably this is easier when $\bar{\kappa}<0$, hence at strong segregation systems, and suppressed otherwise. This reasoning may explain why for some liquids one can manipulate the splashing by an external pressure [69, 70]. Such effects may find applications in various industrial process [71, 72]. Our results may also have implications in emulsion droplet formation as the ease by which drops form might be manipulable by the sign and size of $\bar{\kappa}$.

We have proved that the fluctuations from $\mathrm{L} / \mathrm{L}$ interface away from the planar interface indeed cost free energy. We have shown that the cross-over length has a non-monotonous behavior in the transition regime between weak and strong segregation. Besides this, $\lambda$ is essentially constant (of the order of a few segments lengths) and does not vary much with chain length and/or distance to a critical point. Moreover, a sign-switch of $\bar{\kappa}$ is now established. As interfaces are omnipresent, it is difficult to overestimate the many implications of our phenomenal results which may include complex phenomena such as droplet nucleation from a supersaturated solution, emulsion formation and wetting phenomena to mention a few. 


\section{Additional Information}

The equilibrium properties of symmetric $\mathrm{L} / \mathrm{L}$ interfaces is solved in a mean field model. Solutions of the SF-SCF equations lead not only to thermodynamic information on interfaces but also provides insight in corresponding structural properties. In the first section of this supplementary information we illustrate this by focusing on a few density profiles across symmetric interfaces and then illustrate how curvature of the interface changes the interfaces in a subtle yet expected ways. In a second paragraph we present result for the thermodynamic and elastic properties of interfaces between monomeric solvents (the van der Waals case). In a third section the finite chain length corrections of the interfacial tension as well as the mean bending rigidity are analysed to underpin how the length scale $\lambda=\sqrt{\kappa / \gamma}$ has a non-monotonous behaviour in-between the weak and strong segregation regimes. The fourth section gives information on the interfacial width and the density difference across the interface as functions of the interaction parameter. The final section is devoted to the comparison of the SF-SCF results with the classical results of Matsen [35]. In this comparison we modified the SF-SCF approach by ignoring the non-local interactions in the segment potential. The match of results of these two approaches proves that the difference found between full SF-SCF and the classical SCF used in polymeric interfaces must be attributed to these non-local interactions and not to, e.g., spurious numerical issues.

\section{On the segment density profiles across symmetric interfaces and how curvature of the interface modulates these.}

The SF-SCF theory has been outlined in the chapter. A key result of the theory is the structure of the interface. This interface readily forms both for monomeric systems $A_{1}-B_{1}$ (referred to as the van der Waals interface), as well as for polymeric interfaces $A_{N}-B_{N}$ where $N$ is the degree of polymerisation. The reason why in monomeric systems the interface is stabilized is due to the non-local interactions in the segment potential. For polymer systems the interface in principle should also experience such Cahn-Hilliard gradient contributions (leading to a $k(\Delta \varphi)^{2}$ in the Landau free energy), but they are already stabilized by a Lifshitz-Edwards entropy $\left(b^{2}(\Delta \sqrt{\varphi})^{2} / 12\right.$ in the Landau free energy [57]). Because of the latter effect, in polymeric models the Cahn-Hilliard contribution is often ignored. We show that this Ansatz is leading to wrong predictions for the bending rigidities and this is in more depth elaborated on in the final section of this supplementary information.

In Fig. 2.4a the volume fraction profiles are presented for simple planar monomeric Liquid-Liquid interface at strong segregation (orange) and weak 
segregation (cyan). Interfacial profiles converge to the classical result $\varphi(z)=$ $0.5 \pm \Delta \varphi \tanh \left(\left(z-z^{*}\right) / W\right)$ in the limit of weak segregation. Here $z=z^{*}$ is the position of the interface, $W=b \sqrt{\lambda \chi / \Delta \chi}$ is the width of the interface $(\lambda=1 / 6$, $b$ is the size of a segment), and $\Delta \varphi$ is the density difference (between binodal concentrations) which near critical is given by $\sqrt{6 \Delta \chi / 16}$. Fig. 2.4a is instrumental in visualizing the length scales involved in the problem. Primary length scale, as observed from the figure is the interfacial width $W$. It is clear that closer to the bulk critical point the interfacial width widens and the density difference decreases as observed [45]. Other length scales associate are the coil size $R_{g}=b \sqrt{N / 6}$ and the segment size $b$. We note that these length scale will play a significant role in polymeric liquid-liquid interfaces and are addressed in the chapter.
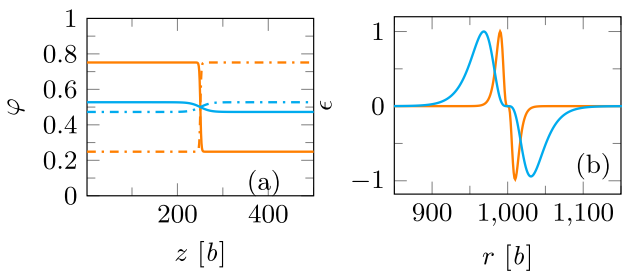

Figure 2.4: (a) Volume fraction profile $\left(\varphi_{A}\right.$ : solid, $\varphi_{B}$ : dashed)for planar liquidliquid interface at strong segregation $(\Delta \chi=0.1$, Orange) and weak segregation $\left(\Delta \chi=0.001\right.$, blue). (Note $\left.\chi^{c}=2\right)$ (b) Corresponding normalized grand potential density for spherically curved interface $\epsilon$ as a function of radial distance. $\epsilon(r)=\frac{\omega_{s}(r)-\omega_{p}(z)}{\max \mid \omega_{s}(r)-\omega_{p}(z)}$. Interfaces are pinned at $r=1000$ and $z=1000$, respectively.

The mechanical parameters of the interface are found by comparing the grand potential of the planar interface with those found in cylindrical or spherical geometry. We use the coordinate $r$ for non-planar geometries and note that $r=0$ is the center coordinate. Upon curving the interface small deviations for the tanh-profiles occur. As a result also the grand potential density profile $\omega(r)$ deviates from the corresponding $\omega_{p}(z)$. In the planar interface $\omega_{p}(z)$ has a maximum positive value at the point where $\varphi(z)=0.5$, i.e. at $z=z^{*}$, and the grand potential density decays exponentially to zero with length scale $\xi$. This function is exactly symmetric meaning that $\omega\left(z^{*}-a\right)=\omega\left(z^{*}+a\right)$ for all values of $a$. In curved geometries this is no longer the case as illustrated in Fig. 2.4b. The grand potential density difference $\epsilon(r)$ (see the legend for the definition of this difference; the absolute value of $\omega$ is 
a strong function of $\Delta \chi$ and therefore we normalised the grand potential density differences so that a comparison is possible) has a positive excursion for $r$-values smaller than the interface position $r=r^{*}$ (here $r^{*}=1000$ ) and a negative excursion for larger $r$. In the weak segregating case the width of the interface is wide and therefore the $\epsilon(r)$-profile varies further away from the position of the interface as well.

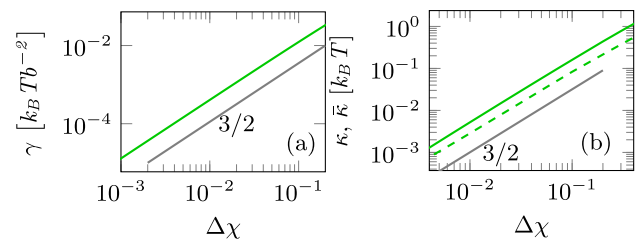

Figure 2.5: (a) Interfacial tension $\gamma$ in units of $\left[k_{B} T b^{-2}\right]$ as a function of $\Delta \chi=\chi-\chi^{c}$ in log-log coordinates (b) Mean $\kappa$ (solid line) and Gaussian bending rigidities $\bar{\kappa}$ (dashed line) in units of $\left[k_{B} T\right]$ as a function of $\Delta \chi$. Relevant slopes are indicated. Results are presented for monomeric $\mathrm{L} / \mathrm{L}$ interface.

\section{Interfacial tension and bending rigidities of monomeric $\mathrm{L} / \mathrm{L}$ interface}

In the chapter we have presented results for the interfacial tension $\gamma$ as well as the bending rigidities $\kappa$ and $\bar{\kappa}$ for symmetric interfaces with molecular weights $N=2$, 20 and 200 as a function of $\Delta \chi=\chi-\chi^{c}$. Similar results for the $N=1$ system, that is for the van der Waals interface, are presented in fig. 2.5. As expected the results are extremely close to those presented in the text for $N=2$ : In good approximation $\gamma \propto(\Delta \chi)^{3 / 2}$. The same power-law dependencies are found for the mean and Gaussian bending rigidities. For all values of $\Delta \chi$ that we can reliably evaluate in the current implementation of the SF-SCF equations (up-to $\chi \approx 2.3$ ), the Gaussian bending rigidity follows the mean bending rigidity. In other words, in this case we do not witness the sign switch for the monomeric system. However, we do expect that for for the van der Waals interface the Gaussian bending will change its sign, but this should happen for $\chi>2.3$. The ratio between $\bar{\kappa} / \kappa \simeq 0.5$ for all values of $\Delta \chi$. A slight deviation in the power law scaling near $\Delta \chi=10^{-3}$ is attributed to computation complications. 


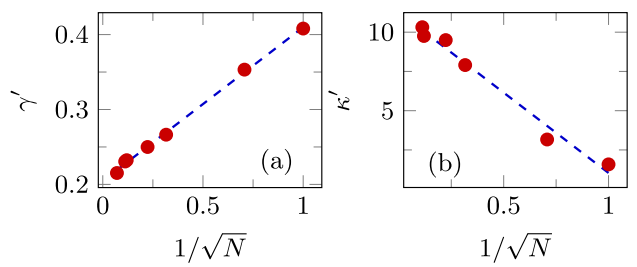

Figure 2.6: (a) $\gamma^{\prime}$ as a function of $1 / \sqrt{N}$. Blue line is a linear fit $\gamma^{\prime} \simeq(0.20+$ $0.20 / \sqrt{N})$ This implies at weak segregation regime $\gamma \simeq\left(0.20+0.20 \frac{1}{\sqrt{N}}\right) N \Delta \chi^{3 / 2}$. (b) $\kappa^{\prime}$ as a function of $1 / \sqrt{N}$. Dashed blue line is a linear fit $\kappa^{\prime} \simeq(11.26-10.22 / \sqrt{N})$. This implies at weak segregation regime $\kappa \simeq\left(11.26-10.22 \frac{1}{\sqrt{N}}\right) N \Delta \chi^{3 / 2}$

\section{Finite chain length effects for interfacial tension and the mean bending rigidity in the weak segregation regime}

In the main text it was shown that in weak segregation, that is for the systems where the interfacial width exceeds the coil size $R_{g}, \gamma \propto N(\Delta \chi)^{3 / 2}$ and $\kappa \propto N(\Delta \chi)^{3 / 2}$. As the ratio $\bar{\kappa} / \kappa=0.5$, the Gaussian bending rigidity follows the same law. In an attempt to find the prefactor (which implements finite chain length effects), we note that the mentioned laws are limiting law that apply for long chains. Inspired by finite chain length corrections known for the interfaces in microphase segregation [65], we consider prefactors of the type $A+B / \sqrt{(} N)$. To this end we define $\gamma^{\prime}=\gamma /\left(N(\Delta \chi)^{3 / 2}\right)$ and similarly $\kappa^{\prime}=\kappa /\left(N(\Delta \chi)^{3 / 2}\right)$ and plot these quantities as a function of $1 / \sqrt{(} N)$ in fig 2.6. Red dots represent the numerical values estimated from SF-SCF calculations. Blue dashed line is a linear fit. As anticipated the linear fit is acceptable and higher order correction of the type $1 / N$ are not needed. small maximum and the height of this maximum is a weakly linear function of the chain length $N$.

Trends observed in Fig. 2.6a, 2.6b prove that for the interfacial tension the prefactor grows with decreasing $N$, that is $\gamma \simeq\left(0.20+0.20 \frac{1}{\sqrt{N}}\right) N \Delta \chi^{3 / 2}$, whereas the negative slope for mean modulus implies that the prefactor decreases with decreasing $N: \kappa \simeq\left(11.26-10.22 \frac{1}{\sqrt{N}}\right) N \Delta \chi^{3 / 2}$. At strong segregation for both $\gamma$ as well as for the bending modulus the proportionality constants are independent of $N$. These results reflect on values of $\lambda=\sqrt{\kappa / \gamma}$. Both limits of $\lambda$ is expected feature finite chain length effects that decay with $1 / \sqrt{N}$ (shown for weak segregation limit in Fig. 4). These corrections obviously become relatively large for $N$ of order unity. We argue that it is reasonable to find that $\lambda$ is of order segment size $b$ : (i) in the 
strong segregation, where both polymers are in the melt state, the $N$-effects are small. (ii) in weak segregation when the interface is much larger than the coil sizes, the properties of the system should be universal, i.e. not dependent on $N$.

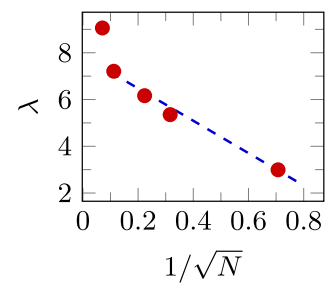

Figure 2.7: Cross-over length $\lambda$ as a function of $1 / \sqrt{N}$ at $\Delta \chi=10^{-3}$ (weak segregation limit). Fit line (blue dashed) shows that $\lambda_{w s}=(7.86-6.92 / \sqrt{N})$ for $N>2$. Fit excludes the $\lambda$ for $N=200$ at $\Delta \chi=10^{-3}$ did not reach the limiting value $\lambda_{w s}$ (cf. Fig. 2a in the main text).
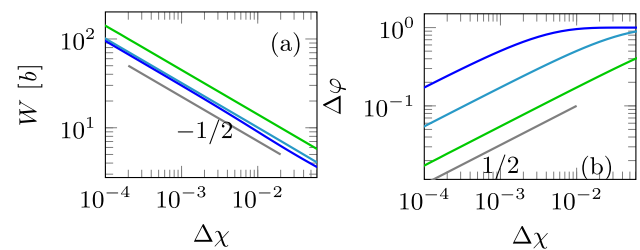

Figure 2.8: (a) Interfacial width and (b) Density difference as a function of closeness to bulk critical point for various $N$. Relevant slopes are indicated.

\section{The interfacial width and the density difference.}

In Fig. 2.8a and 2.8b, the interfacial width and the density difference is presented as a function of $\Delta \chi$ in double logarithmic coordinates for three values of the molecular weights $N=2,20$ and 200. For the weak segregation regime, that is when the width $W$ exceeds the coil size $R_{g}$ we find van der Waals like scaling: $W \propto(\Delta \chi)^{-1 / 2}$ and the density difference obeys to $\Delta \varphi \propto \sqrt{N}(\Delta \chi)^{1 / 2}$ (Figure not shown). The $\sqrt{N}$ in the latter dependence can be understood from the notion that for weak segregation the van der Waals theory suggests $\gamma \propto(\Delta \varphi)^{2} / W \propto N(\Delta \chi)^{3 / 2}$. [45] 
Our interest goes again to the proportionality constants for these scaling dependencies. Interestingly, for the density difference we find for all chain length $\Delta \phi=1$ in strong segregation and in weak segregation the proportionality constant is independent of $N: \Delta \varphi=1.22 \sqrt{N}(\Delta \chi)^{1 / 2}$. For $W$ we have non-trivial prefactors both in the weak as well as in the strong segregation limits. Similarly as for the interfacial tension and the rigidities, we define $W^{\prime}=W /\left((\Delta \chi)^{-1 / 2}\right)$. Using this, we find for weak segregation (cf fig. 2.9a) that the width of the interface obeys $W=(0.94+0.93 / N) \Delta \chi^{-1 / 2}$ while at strong segregation (cf Fig. 2.9b) $W=(0.82+0.84 / \sqrt{N}) \Delta \chi^{-1 / 2}$.

Note that only in the strong segregation case we have the $1 / \sqrt{N}$-type finite chain length correction for $W$. This dependence is consistent with the $N$-dependence for the sign switch of $\bar{\kappa}$ (analysed for sufficiently long chains). This leads us to believe that the width of the interface is leading the sign switch of $\bar{\kappa}$. In the text we mention that the Gaussian bending rigidity changes sign when the width of the interface is three to four times the segment size. Numerically the correspondence between the sign switch and the finite chain length correction for the width $W$ do not exactly match. We argue that this is because the sign switch actually takes place in the cross-over regions, that is for short chains it happens in the weak segregation while for longer chains (most of the systems) it occurs in the strong segregation regime.

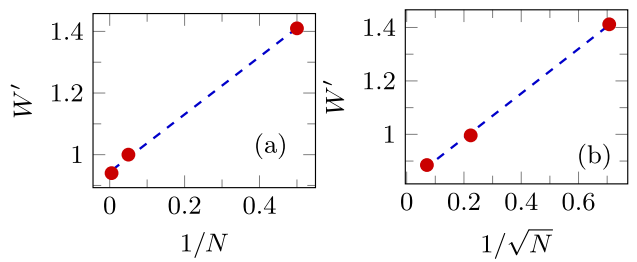

Figure 2.9: (a) $W^{\prime}$ as a function of $1 / N$ in weak segregation $\left(\Delta \chi<10^{-3}\right)$. Dashed blue line is a linear fit $W^{\prime} \simeq(0.94+0.93 / N)$. (b) $W^{\prime}$ as a function of $1 / \sqrt{N}$ in strong segregation $\left(\Delta \chi>5 \times 10^{-2}\right)$ regime. Blue line is a linear fit $W^{\prime} \simeq(0.82+0.84 / \sqrt{N})$.

\section{Interfacial tension and bending rigidities in the absence of non-local interactions.}

The purpose of this section is to prove that the SF-SCF formalism gives essentially the same results as the SCF theory used for microphase segregation, provided that 
in the SF-SCF calculations we introduce the approximation that $\langle\varphi(r)\rangle \rightarrow \varphi(r)$. This approximation is typically used in the SCF theory for microphase segregation, whereas in the SF-SCF theory the non-local interactions are typically taken into account. We focus on the interfacial tension and the binding rigidities for polymeric interfaces as reported by Matsen [35]. We will refer to the latter theory as M-SCF, and use m-SF-SCF for the modified SF-SCF formalism.

Both m-SF-SCF as M-SCF need to evaluate the chain partition functions. In both approaches the Edwards diffusion equation is evaluated:

$$
\frac{\partial G}{\partial N}=\frac{1}{6} \nabla^{2} G-u G
$$

In the $\mathrm{m}$-SF-SCF this equation is mapped on a lattice using a finite difference approach. The polymers are taken to be built up by segments with ranking numbers $s=1,2, \cdots, N$ and the space is discretized by a lattice. The lattice site size is taken identical to the segment size so that there is a single length scale $b$ in the problem. The Edwards equation on the lattice results in so-called propagators, which formally implies a change of the chain model from a Gaussian chain (Edwards equation) to a freely jointed chain model with a limited number of step directions. As long as the chains are not strongly stretched the difference between the Gaussian chain model and the freely jointed chain can be ignored.

In the M-SCF approach the discretization is done using a finite elements approach. Formally the walk along the contour is arbitrarily split up in short contour elements, the number of these elements is independent of the real chain length $N$; the idea is that the chain is seen as an thin featureless thread. In doing so, the results are valid in the infinite chain length limit and the results are typically presented with $\chi N$ as a single parameter. We therefore should expect that m-SF-SCF and M-SCF can only give corresponding results when in m-SF-SCF the chain length is sufficiently large. Without mentioning otherwise we have implemented $N=200$. Note that in m-SF-SCF the theory will fail to model L/L interfaces in the limit of $N \rightarrow 1$.

Both approaches need an iterative method to find the SCF solutions. The 'kitchen' of how this is precisely done is not the topic of this comparison. Assuming that this iterative method is done with the appropriate accuracy, the different approaches should not be relevant. Indeed the good comparison shown below implies that both approaches did what they claimed to do, namely that the accuracy of the SCF solution is sufficient to find numerically accurate results for the mean and Gaussian bending rigidities as well as for the interfacial tension. 


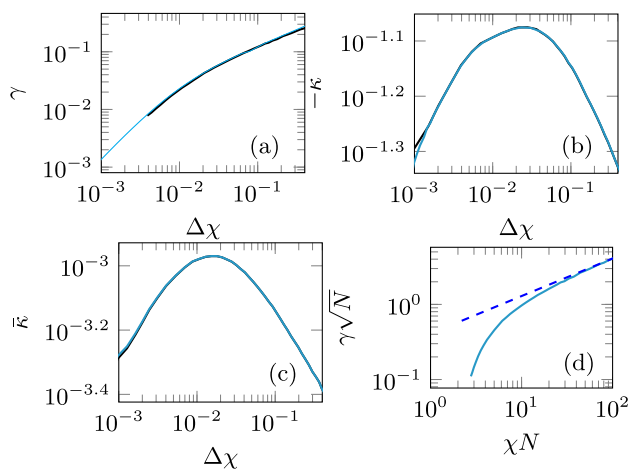

Figure 2.10: Comparison of SCF results for polymeric interfaces when in the SCF theory non-local interactions are not taken into account: (Black line) M-SCF $(N=200)$, (Cyan line) m-SF-SCF. (a) The interfacial tension in $k_{B} T / b^{2}$. (b) Mean bending rigidity in units of $k_{B} T$ (c) Gaussian bending rigidity in units $k_{B} T$ as a function of $\Delta \chi$. (d) Comparison of interfacial tension with analytical strong segregation theory: $\gamma \sqrt{N}$ as a function of $\chi N$. All quantities are plotted in double logarithmic coordinates. All graphs are inspired by similar graphs found in ref [35] from where also the M-SCF results were extracted.

In Fig. 2.10 we present the comparison of results for the interfacial tension and the bending rigidities for the $\mathrm{m}-\mathrm{SF}-\mathrm{SCF}$ and the M-SCF theories. When we discuss these results we will contrast these with the full SF-SCF results that are presented in the chapter.

In Fig. 2.10a we show results for the interfacial tension. As can be seen both approaches match accurately. The power-law scaling at large $\Delta \chi$ reveal the $1 / 2$ slope which is also found in the complete Sf-SCF theory. For weak segregation it is found that $\gamma$ decreases steeper than the $1 / 2$ scaling. Unlike in full SF-SCF where the slope of $3 / 2$ is found, here the results are more leaning to the slope of unity. The fact that near the critical point the mean field results are not expected to be accurate, explains why not much attention was given to the weak segregation results in the microphase segregation community. In Fig. 2.10d it is shown that for strong segregation the numerical m-SF-SCF as well as the M-SCF approach the strong segregation limiting law predicted by analytical theory (dashed line).

In Fig. 2.10b we present results for the mean bending rigidity as a function of $\Delta \chi$ in double logarithmic coordinates. Note the negative sign along the y-axis. Indeed, both in m-SF-SCF as well as in M-SCF the mean bending modulus is negative for all values of $\Delta \chi$. Also in both approaches $-\kappa$ is non-monotonic: the 
absolute value first increases and then decreases with increasing $\Delta \chi$. These results are in stark contrast with full SF-SCF results for which the mean bending modulus is positive and grows monotonically with $\Delta \chi$, showing two regions of scaling with slopes $3 / 2$ and $1 / 2$ for weak and strong segregation, respectively.

The Gaussian bending rigidity is presented in Fig. 2.10c. Again m-SF-SCF and M-SCF are in full agreement: $\bar{\kappa}$ is positive for all values of $\Delta \chi$. Similarly as for the mean bending rigidity a non-monotonic dependence is found. The ratio $\bar{\kappa} / \kappa$ is a function of $\Delta \chi$. Again the results are in strong conflict with the full SF-SCF ones for which a sign switch was predicted. Moreover in full SF-SCF both in weak and strong segregation the ratio $\bar{\kappa} / \kappa$ was shown to assume fixed values of 0.5 and -1.5 , respectively. These fixed ratio's do not occur in the M-SCF results.

Conclusions regarding the comparison. (i) We have shown that m-SF-SCF predictions for for the interfacial properties of polymeric interfaces did not significantly deviate from the M-SCF predictions. Both approaches use the same way to pin the interface to a specified location which allows the bending at fixed chemical potentials, both approaches use the same Edward diffusion equation and both approaches solve the equations numerically accurate. We see this as a support that also the full SF-SCF results are numerically accurate. (ii) The difference between full SF-SCF and the m-SF-SCF/M-SCF results is large. Results not only differ quantitatively, even qualitatively they do not match. The sign of the bending modulus differs. The functionality with $\Delta \chi$ is completely different. The same applies for the Gaussian bending rigidity. In the full calculations we see a sign switch, which appears to be absent in the m-SF-SCF/M-SCF results. (iii) In full SF-SCF the length scale $\lambda=\sqrt{\kappa / \gamma}$ with a clear physical interpretation, follows meaning-full trends. In m-SF-SCF as well as in M-SCF the value of $\lambda$ can not even be computed because $\kappa$ is negative, and hence $\lambda$ is meaningless. (iv) As the only difference between full SF-SCF and m-SF-SCF rests in the approximation $\langle\varphi(r)\rangle \equiv \varphi(r)$, we now argue that $\langle\varphi(r)\rangle$ can not be approximated by $\varphi(r)$. Nonlocal contributions are essential to find accurate predictions for the (mean field) results near the bulk critical point (for all quantities) and importantly for the mean and Gaussian bending rigidities at strong segregation. 


\section{CHAPTER 3}

\section{Liquid-Liquid-Surfactant interfaces: Route to estimate rigidities}

Bending rigidities of tensionless balanced liquid-liquid interfaces as occurring in microemulsions are predicted using self-consistent field theory for molecularly inhomogeneous systems. Considering geometries with scale invariant curvature energies gives unambiguous bending rigidities for systems with fixed chemical potentials: The minimal surface Im3m cubic phase is used to find the Gaussian bending rigidity, $\bar{\kappa}$, and a torus with Willmore energy $W=2 \pi^{2}$ allows for direct evaluation of the mean bending modulus, $\kappa$. Consistent with this, the spherical droplet gives access to $2 \kappa+\bar{\kappa}$. We observe that $\bar{\kappa}$ tends to be negative for strong segregation and positive for weak segregation; a finding which is instrumental for understanding phase transitions from a lamellar to a sponge-like microemulsion. Invariably, $\kappa$ remains positive and increases with increasing strength of segregation. 


\subsection{Introduction}

Interfaces characterized by dense surfactant packings, such as microemulsions [73-75] and biological membranes [76] that are found naturally or manipulated artificially to be in a state of near-zero tension, have extensive areas. Often such interfaces feature a spontaneous curvature that manifests in spherical or cylindrical (swollen) micelles $[77,78]$. When a system is tensionless and precisely balanced-typical for single component bilayers and expected for the middle-phase microemulsions - the interface's spontaneous curvature vanishes [45] and ultra-low interfacial energies can be achieved $[79,80]$. Here, the elastic moduli, mean $(\kappa)$ and Gaussian $(\bar{\kappa})$ bending rigidities, control the interface fluctuations and topology, respectively. Such systems show a first-order phase transition from lamellar to sponge-like phases, e.g., upon an increase of the temperature for nonionic systems, and a change of the salinity for ionic systems $[75,79,81]$. A pre-eminent challenge is to predict, from a molecular model for such interfaces, a means to induce a sign change in the $\bar{\kappa}$ from negative to positive; this signals the loss of stability of the lamellar, $L_{\alpha}$, oil-surfactant-water ordering in favor of a phase with saddles, $L_{3}$ or sponge-like. Another long-standing problem is understanding the relation between surfactant chain architecture and corresponding bending rigidities $[82,83]$.

Earlier theoretical methods [84, 85], experiments [86-88], and simulations [89, $90]$ that attempted to link bending rigidities to molecular properties did not provide information on $\bar{\kappa}$; moreover, the results for $\kappa$ were not consistent with each other. Therefore uncertainties prevail and these persist also because internal checks for presented rigidities are rarely provided. As a result, there exists no accepted molecular level theory that convincingly links molecular characteristics to both mechanical parameters of the interfaces $(\kappa$ and $\bar{\kappa})$. Notably, the missing information for $\bar{\kappa}$ is remarkable as its magnitude and, in particular, its sign are fundamental to the understanding of microemulsions.

The primary obstacle in establishing a molecular model for determining bending rigidities is the requirement of curving the interface at fixed chemical potentials. In this letter, we propose an elegant protocol with internal checks to find these rigidities. We consider interfaces with scale invariant curvature energies and illustrate the protocol for tensionless, balanced liquid-liquid (L/L) interfaces. In line with experimental findings, we report the existence of a sign switch for $\bar{\kappa}$ which triggers a phase transition from planar to sponge-like phases in middle-phase microemulsions. We focus on the role of the interaction parameter which in strong segregation has a large value and for weak segregation a small value; further, we elaborate on the role of the molecular weights of the solvents and that of the amphiphile.

Experiments, simulations, and calculations [84-90] reviewed above have major disadvantages and ambiguities because the systems featured too many complications. We examine a tensionless balanced interface which still is highly relevant to middle- 
phase microemulsion systems wherein oil and water are separated by a surfactant film with extensive areas and often a complex interface topology. Our focus on tensionless (interfacial tension $\gamma=0$ ) balanced (spontaneous curvature $J_{0}=0$ ) $\mathrm{L} / \mathrm{L}$ interface avoids the complications of a finite Laplace pressure (i.e. $\Delta P_{L}=0$ ) when imposing some interfacial curvature. Such a model is readily implemented in the Scheutjens-Fleer Self-Consistent Field theory (SF-SCF) for molecularly inhomogeneous systems. We can consider this idealized system in three different geometries with scale-invariant curvature energies. The latter is essential, as it allows for an analysis in the grand canonical ensemble $(\mu, V, T)$, which opens a convincing route to estimate the rigidities: (i) A spherically curved droplet with $\Delta P_{L}=0$ is used to find $2 \kappa+\bar{\kappa}$; (ii) A minimal Im3m surface (by construction has $\Delta P_{L}=0$ ) is used to find $\bar{\kappa}$; (iii) A minimal torus interface is used to find $\kappa$ also for conditions that $\Delta P_{L}=0$.

We note that the route to obtain rigidities of balanced tensionless $\mathrm{L} / \mathrm{L}$ interfaces shows similarities but also important differences from the symmetric freely dispersed lipid bilayers [55]. For bilayers, we could use the Im3m cubic phase and the spherical vesicle to find $\bar{\kappa}$ and $2 \kappa+\bar{\kappa}$, respectively. The cylindrically curved vesicle could be used to obtain $\kappa$ in two ways: (i) As the number of lipids per unit area is found to be a constant (i.e., not a function of the radius $R$ of the cylindrical vesicle), $\kappa$ was found from the excess Helmholtz energy per unit length, $F_{c}^{\sigma}$, i.e., $\kappa=R F_{c}^{\sigma} / \pi$; (ii) Realizing that the grand potential of the cylindrical vesicle per unit length $\Omega_{c}$ is split up equally into bending energy and stretching energy, $\kappa$ is also found from (half) the grand potential density per unit length, i.e. $\kappa=R \Omega_{c} /(2 \pi)$. However, for the tensionless balanced L/L interface, curved in cylindrical geometry with $\Delta P_{L}=0, \kappa$ can neither be computed from the Helmholtz energy per unit length, nor from the grand potential per unit length, as there is neither a conservation of the number of surfactant per unit area nor a conservation of the chemical potentials of the molecules of the system, cf. Figs. (3.1c) and (3.1d) shown below. Importantly, in the $\mathrm{L} / \mathrm{L}$ interface, we do not find a coincidental equal splitting of curvature and tension energies.

Following Helfrich, by expanding the interfacial tension $(\gamma)$ in mean curvature $\left(J=1 / R_{1}+1 / R_{2}\right)$ and Gaussian curvature $\left(K=1 / R_{1} R_{2}\right)$, with $R_{1}, R_{2}$ being principle radii of curvature as

$$
\gamma(J, K)-\gamma(0,0)=-\kappa J_{0} J+\frac{1}{2} \kappa J^{2}+\bar{\kappa} K,
$$

we identify $\gamma(J, K)$ as the appropriate characteristic function that carries the bending information for curving the interface at constant chemical potentials [47]. This expansion is the starting point for our analysis of interfacial equilibrium properties, as it appears, refer Fig. (3.1d), for the balanced L/L interface, we find curved interfaces that exist at chemical potentials equal to that of the ground state (tensionless balanced planar interfaces) not only for the surfactant but also for the 

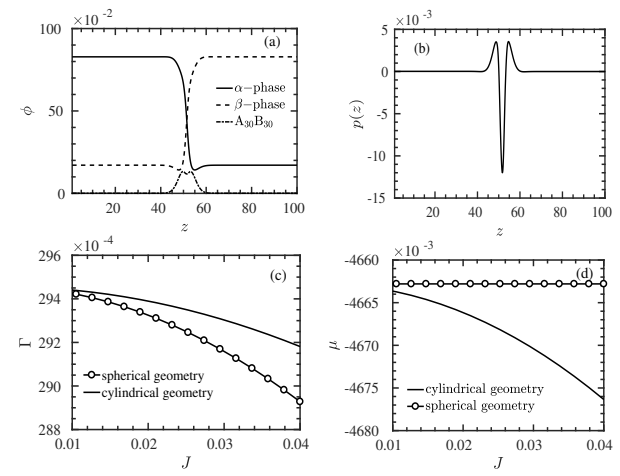

Figure 3.1: (a) Volume fraction distribution and (b) Lateral pressure distribution (in $k_{B} T / b^{3}$ ) in a planar tensionless interface. (c) Area per surfactant molecule $(\Gamma)$ [in units $b^{2}$ ] and (d) Chemical potential of surfactant $\left(\mu \equiv \mu_{s}\right)$ [in units of $k_{B} T$ ] as a function of interface curvature as indicated for systems with $\Delta P_{L}=0$. Surfactant $\mathrm{A}_{30} \mathrm{~B}_{30}$, solvents, $\mathrm{A}_{4}$ and $\mathrm{B}_{4} \cdot \chi=0.6$

two solvents. Note, that in this system, both $\gamma(0,0)=0$ and $J_{0}=0$, Eqn. (3.1) simplifies to $\gamma(J, K)=\frac{1}{2} \kappa J^{2}+\bar{\kappa} K$.

\subsection{Method and Model}

Within SF-SCF framework, extremizing the mean field free energy for a molecularly inhomogeneous system provides both structural and accurate thermodynamic information [53, 55-57, 91-93]. We have implemented a coarse-grained molecular model in which there are two types of spherically symmetric segments A and B. These segments are used in two solvents, each with length $n, A_{n}$ and $B_{n}$ forming the two liquid phases $\alpha$ and $\beta$, respectively, and in a diblock copolymer composed of blocks of equal length $N, A_{N} B_{N}$. This approach requires molecular partition functions, which are evaluated within a lattice considering the molecules as freely jointed chains. Accordingly, segments fit on lattice sites. The lattice sites are organized as homogeneously curved- or planar layers. Driven by the segregation between the segments, an interface develops on which the lattice geometry imposes the curvature. Segment density gradients can only develop in the direction perpendicular to such interface, as a mean field approximation is implemented in lattice layers 'parallel' to the interface. In the absence of density gradients, the model is equivalent to the Flory-Huggins theory. There is just one Flory-Huggins interaction parameter $(\chi)$ between monomers A and B. We choose a value slightly above the critical point of the binary solvent $\left(\chi^{c r}=2 / n\right)$. Below the minimum value used for $n$ is 4 , and 
the interaction is chosen between $\chi=0.52-0.68$ (for more details on method and model refer supplemental material [59]).

Volume fraction profiles, $\varphi(z)$, and the lateral pressure distribution, $p(z)=$ $-\omega(z)$, with $\omega$ as the grand potential density, are presented for the default planar tensionless L/L interface, in Figs. (3.1a) and (3.1b). Here, $z \equiv z / b$ is the dimensionless normal coordinate. In Fig. (3.1a) we see that the two liquids give a Van der Waals-like profile and the accumulated copolymers have their blocks on corresponding sides of the interface. The pressure profile $p(z)$, see Fig. (3.1b), has a negative excursion at the interface due to the contribution from the $\mathrm{L} / \mathrm{L}$ interface and positive 'wings' on either side of the interface due to the overlap of copolymers in a brush-like configuration. From earlier work [94], we know that $\gamma=-\sum_{z} p(z)$ and that the second moment of the pressure distribution with respect to the Gibbs plane $\left(R^{g}\right)$ provides a direct estimate of $\bar{\kappa}=\sum-\left(z-R^{g}\right)^{2} p(z)$. The latter relation proved useful for the evaluating $\bar{\kappa}$ of lipid bilayers, and presents a strong test for alternative, more elaborate routes to obtain the same quantity.

Similar as for lipid bilayers, an independent alternative route for evaluating $\bar{\kappa}$ makes use of three-gradient SCF computation as shown in Fig. (3.2a), where on all six faces of the elementary box, Neumann boundary conditions apply; the elementary box is $1 / 8$ th of a unit cell of an Im3m phase, 8 unit cells are shown in Fig. (3.2b). When equal amounts of A and B are present in the system, the interface splits the volume into two identical sub-volumes (phase $\alpha$ and $\beta$ ) while $J=0$ along the surface and $\Delta P_{L}=0$. As soon as the copolymers are added, such that the chemical potential of all molecular species is equal to the corresponding values of the planar tensionless system, we lie within an $(\mu, V, T)$-ensemble; thus, the grand potential, $\Omega=F-\sum_{j} \mu_{j} n_{j}$, is the characteristic function, and $\Omega=\bar{\kappa} \int_{M} K d A$. Using Gauss-Bonnet theorem for compact, boundary-less Riemann manifold, the integral of curvature over the area can be evaluated as $-8 \pi[95,96]$. Thus, the grand potential for the unit cell, $\Omega=-8 \pi \bar{\kappa}$. Hence, from the scale-invariant grand potential $\Omega$ directly follows $\bar{\kappa}$. The result is consistent with the second moment over the pressure profile (see Tab. (1) in supplemental material [59]).

The procedures to evaluate $\kappa$ are more involved. In Figs. (3.1c) and (3.1d), we have presented typical results for spherically and cylindrically curved interfaces when $\Delta P_{L}=0$ as a result of the adsorption of the copolymers. In Fig. (3.1c) we show the area per copolymer at the interface (inverse of the adsorbed amount) and in Fig. (3.1d) the corresponding chemical potentials as a function of the curvature $J$. In Fig. (3.1d) we notice that the chemical potentials remain constant upon bending in case of spherical curvature. This means that in this geometry bending is performed in the $(\mu, V, T)$-ensemble. The reason why the system can maintain its chemical potentials upon bending of the interface is traced to the known fact that integrating Eqn. (3.1) over the area, $\Omega=\int_{M} \gamma(J, K) d A=4 \pi(2 \kappa+\bar{\kappa})$, is a constant irrespective of the size of the spherical droplet showing scale invariance. 


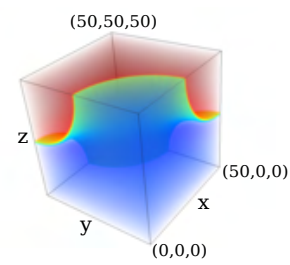

(a)

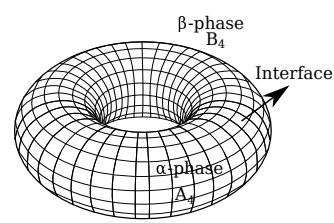

(c)

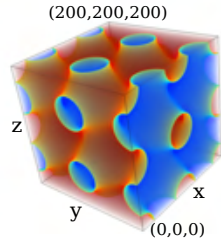

(b)

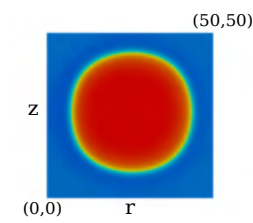

(d)

Figure 3.2: Volume fraction distribution of $\alpha$-phase from 3D SCF calculation of interface modeled as Im3m cubic phase. 1/8 of a unit cell is shown in (a) 8 unit cells are shown for visualization in (b). Schematic illustration of an interface in torus shape is shown in (c). Volume fraction distribution of $\alpha$-phase from 2D SCF calculation of minimal torus in a cylindrical lattice is shown in (d). The molecular model is similar as in Fig. 3.1. Color scale from blue to red is $0.2-0.8$ for all contours.

Now an indirect route is available to compute $\kappa$, namely from combining the total curvature energy from the spherical droplet with the Gaussian bending modulus $\bar{\kappa}$ found above. Ideally, we would like to validate this indirect route with a direct estimate.

Again, as in the cylindrical geometry, neither the adsorbed amount of surfactant, cf. Fig. (3.1c), nor the corresponding chemical potential, cf. Fig. (3.1d), is conserved, and we cannot use this geometry to obtain $\kappa$. A direct route to evaluate the mean bending modulus is still possible using a system that features a minimal torus, as illustrated in Fig. (3.2c). Within SF-SCF this is realized using a twogradient $(r, z)$ cylindrical lattice. A typical result is presented in Fig. (3.2d) as a density contour plot in the $(r, z)$ cross-section. From Gauss-Bonnet theorem, as the torus has genus $g=1$ the integral $\int_{M} K d A$ vanishes. Moreover, the socalled Willmore energy of the torus has contribution only from mean curvature, $W=\frac{1}{4} \int_{M} J^{2} d A$.

In 1965, T.J. Willmore conjectured that the Willmore energy $(W)$ of a smooth torus immersed in $3 \mathrm{D}$ space is always greater than or equal to $2 \pi^{2}$ [97]. This conjecture was proved by Marques and Neves in 2012 [98]. The Willmore energy reaches its minimum when the radius of revolution is $\sqrt{2}$ times the radius of the 
generating circle, as shown in Fig. (3.2d). By integrating the Helfrich equation for the toroidal configuration with minimal Willmore energy, we obtain the grand potential for torus as, $\Omega_{t}=\frac{1}{2} \kappa \int_{M} J^{2} d A=2 \kappa W=4 \pi^{2} \kappa$.

Now the protocol boils down to generating this minimal torus in SF-SCF while adding the copolymer such that $\Delta P_{L}=0$. It occurs that in this case, the system converges with all its chemical potentials equal to that of the planar tensionless interface and lies within the $(\mu, V, T)$-ensemble. Similar as in the droplet case this result is traced to the scale invariance, in this case of the minimal Willmore energy. Its grand potential gives a direct estimate of $\kappa=\Omega_{t} / 4 \pi^{2}$.

The values found for $\kappa$ by the direct and indirect routes are congruent, proving that there is complete consistency in obtaining the bending rigidities, using scaleinvariant surfaces, for tensionless balanced L/L interfaces [59]. Our protocol is available at https://wp.me/p7KmNt-9C as an open-source software package.

\subsection{Results}

As we have established the molecular link for bending rigidities, we now present the chain length dependence of the bending rigidities for the regime where $N>n$ in in Fig. (3.3a) and (3.3b), and for $N \approx n$ in Fig. (3.3c) and (3.3d). The trends for $N>n$ support the results from simulations $[89,90,99]$. While it would be alluring to conclude that bending rigidities have a linear dependence on the chain length of the surfactants, results in regime $n \approx N$ contradict this observation and the dependences are clearly nonlinear, see Figs. (3.3c) and (3.3d). It is observed that the dependence of rigidities on surfactant chain length is strongly influenced by solvent chain length and interaction parameter between monomeric units, an important effect which has not been addressed in previous works [82-90]. A thorough analysis of the magnitude of $\kappa$ is, however, beyond the scope of the present letter and will be presented elsewhere.

Moving from the regime where the solvent length is smaller compared to the surfactant block length, $n<N$, to the regime where the solvent length is comparable to that of the surfactant, we observe that $\bar{\kappa}$ is of opposite sign, cf. Figs. (3.3a) and Fig. (3.3c). Such a sign switch is of exceptional interest, as it addresses a topological phase transition in microemulsions that can be achieved in two ways:

(1) by tuning the interaction parameter for fixed solvent and surfactant lengths and

(2) by tuning solvent length for fixed surfactant length and $\Delta \chi=\chi-2 / n$.

In Fig. (3.4a) the dependence of both $\kappa$ and $\bar{\kappa}$ are shown for surfactant block length of $N=20$ as a function of a measure of closeness to the critical point of the binary solvent $\Delta \chi$. The Gaussian bending modulus, $\bar{\kappa}$, switches from negative to positive when moved towards weak segregation; this transition occurs earlier in higher $n$ (dashed line) for fixed $N$. 

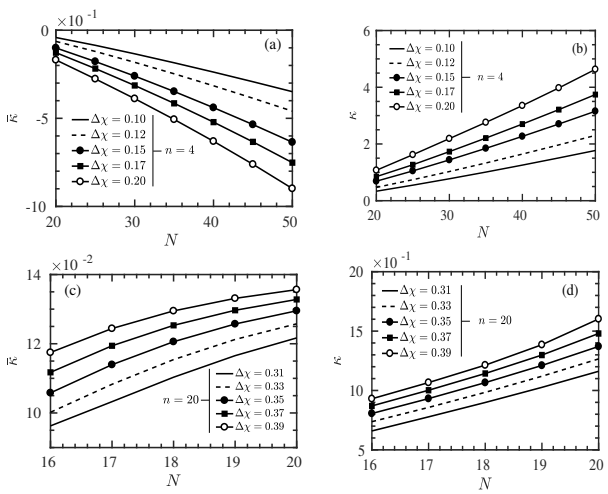

Figure 3.3: Chain length dependence of bending rigidities [in units of $k_{B} T$ ]. (a) and (b) $N>n$ Regime: Chain length of bulk phases fixed $\left(\mathrm{A}_{4}, \mathrm{~B}_{4}\right)$, surfactant chain length is varied $\left(\mathrm{A}_{N} \mathrm{~B}_{N}\right.$, where $\left.20<N<50 ; 0.1<\Delta \chi<0.2\right)$. (c) and (d) $N \approx n$ Regime: Chain length of bulk phases fixed $\left(\mathrm{A}_{20}, \mathrm{~B}_{20}\right)$, surfactant chain length is varied $\left(\mathrm{A}_{N} \mathrm{~B}_{N}\right.$, where $\left.16<N<20 ; 0.3<\Delta \chi<0.4\right)$.

A similar effect can also be achieved by tuning the surfactant chain length for a given solvent chain length and interaction energy $(\Delta \chi)$. Experimentally, one can reach weak segregation by the addition of a suitable co-solvent which diminishes the difference between the two primary solvents.

A summary of results, obtained by tuning $N$, is presented as a 'phase diagram' in Fig. (3.4b). The two governing parameters, i.e., block length of the copolymer and the chain length of the solvent are on the $x$ - and $y$-axis, respectively. The interaction parameter is chosen as $\Delta \chi$. By tuning the surfactant length for given interactions $(\Delta \chi)$ and solvent chain length, $\kappa$ increases and $\bar{\kappa}$ decreases monotonically, also showing a sign switch at the solid lines.

These results imply the tendency of the interface to remain planar on average when $n<<N$. This result is contrasted with the situation when the length of the solvent molecules is increased to be similar, $n \approx N$, or even larger than that of the copolymer, $n>N ; \bar{\kappa}$ becomes positive in this regime while $\kappa$ is small but positive. For large $n$, we have $\bar{\kappa}>0 ; 0<\kappa<1$. These features are consistent with a sponge phase (Winsor III) [78] which grows in importance with reducing $\chi$. Stable but very flexible, and strongly fluctuating lamellar phases (as $\bar{\kappa}<0$ and $0<\kappa<1$ ) are observed as $N$ is increased for fixed $n$, whereas for very large $N, \kappa$ is $>1$, and we enter a region where the fluctuations of the interface are weak, crossing the dashed lines, as shown in Fig. (3.4b). 


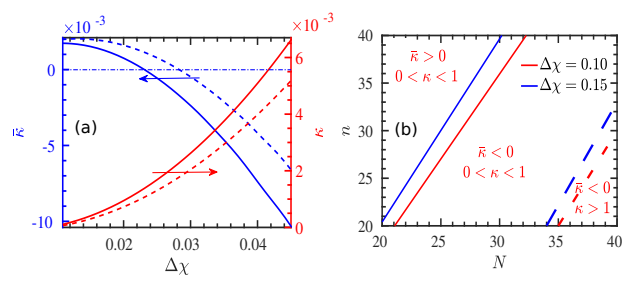

Figure 3.4: (a) Gaussian bending modulus (blue axis) $\bar{\kappa}$ [in units of $k_{B} T$ ] and mean bending modulus (red axis) $\kappa$ [in units of $k_{B} T$ ] as a function of $\Delta \chi(\Delta \chi=\chi-2 / n)$. Surfactants are modeled as $A_{N} B_{N} . \alpha$-phase is modeled as $A_{n}$ and $\beta$-phase is modeled as $B_{n}$. [solid line: $n=4$, dashed line: $n=6$ ] (b) Phase diagram in $n$ and $N$ coordinates for fixed $\Delta \chi$ as indicated. The sign and magnitude [in units of $k_{B} T$ ] of the rigidities are indicated. Note that within the mean field model $n, N$ are related to the radii of gyration in the bulk as $R_{g}=b \sqrt{n / 6}$ ) and $R_{G}=b \sqrt{2 N / 6}$, for the solvent and copolymer respectively, where $b$ is bond length.

\subsection{Summary}

We have linked molecular characteristics to bending rigidities for surfactant-covered $\mathrm{L} / \mathrm{L}$ interfaces. Using surfaces with scale-invariant curvature energies is embellished as an elegant route to determine $\kappa$ and $\bar{\kappa}$ unambiguously; this route cautiously exploits the tensionless state of the interfaces and avoids the linear term in curvature. Large deviations from these constraints imply the loss of the microemulsion middlephase in favor of emulsions with oil-in-water or water-in-oil droplets; however, understanding the effects of small deviations is vital, as it is a prerequisite for any detailed comparison with experiments. The current analysis provides a natural starting/reference point to generalize for molecular asymmetry, spontaneous curvature and finite tension of the interfaces. 


\section{CHAPTER 4}

\section{Liquid-Liquid-Surfactant interfaces at strong and weak segregation limit}

Microemulsions are explored using the self-consistent field approach. We consider a balanced model that features two solvents of similar size and a symmetric surfactant. Interaction parameter $\chi$ and surfactant concentration $\varphi_{s}^{b}$ complement the model definition. The phase diagram in $\chi-\varphi_{s}^{b}$ coordinates is known to feature two lines of critical points, the Scott- and Leibler line. Only upon imposing a finite distance between the interfaces we observe that the Scott line meets the Leibler line. We refer to this as a Lifshitz point (LP) for real systems. We add regions that are relevant for microemulsions to this phase diagram by considering the saturation line, which connects $\left(\chi, \varphi_{s}^{b}\right)$-points for which the interface becomes tensionless. Crossing this line implies a first-order phase transition as internal interfaces develops, characteristic for one-phase microemulsions. The saturation line ends at the socalled microemulsion point (MP). MP is shown to connect with LP by a line of MP-like critical points; found by searching for a ' $\mathrm{MP}$ ' while the distance between interfaces is fixed. A pair of binodal lines that envelop the three-phase (Winsor III) microemulsion region, is shown to connect to MP. The cohesiveness of the middle phase in Winsor III is related to non-monotonic, inverse DLVO type interaction curves between the surfactant-loaded tensionless interfaces. The mean and Gaussian bending modulus, relevant for the shape fluctuations and the topology of interfaces, respectively, are evaluated along the saturation line. Near the MP both rigidities are positive and vanish in a power-law fashion with coefficient unity at the MP. Overseeing these results proves that the MP has a pivoting role in the physics of microemulsions. 


\subsection{Introduction:}

Microemulsions are thermodynamically stable ternary mixtures of oil, water and surfactants that feature enormous amounts of internal interfaces $[4,100,101]$. Often, microemulsions consist of small droplets (of oil in water or water in oil). A co-solvent may be added to reduce the difference between oil and water, suppressing or in some case preventing the formation of droplets. In such scenario the interfacial tension may reach ultra-low values[102] and the microemulsion topology is lamellar or sponge like[80, 103]. The close proximity of complementary solvents in such systems is rather unique and based on this there exist many important applications in many disciplines, e.g., ranging from cleaning to cooking, from formulation science to pharmacy and cosmetics[104-107]. Therefore there is a strong need to know more about the physical properties of microemulsions and gain deeper insight in the structure and thermodynamics of these complex solutions.

To address this need, we use the lattice based self-consistent field theory of Scheutjens and Fleer (SF-SCF) $[53,54,57]$ and aim to find thermodynamic and structural information of interfaces relevant for microemulsions. In this approach the molecules are considered as strings of equally sized segments with either a polar or an apolar nature. This theory is constructed around the Flory-Huggins equations of state[108]. It is thus assumed that the system is incompressible and interactions are short ranged, specified by exchange interaction parameters $\chi$. Within this framework we are going to work with a minimal model for microemulsions, which contains three components. More specifically, our focus is on balanced microemulsions as the corresponding model has a minimum number of parameters. Moreover, in a balanced model we can safely ignore the formation of droplets and, as we will argue, the microemulsification (formation of internal interface e.g. upon an increase of the surfactant concentration) occurs as a true first order transition. As a consequence, the microemulsions that are considered cannot have droplets and must be of a lamellar or sponge-like nature.

To facilitate the following, it is convenient to specify our model more precisely. In the model we consider a pair of solvents $A_{n}$ and $B_{n}$. Hence both solvents have the same size $n$. At all times we will have equal amounts of these solvents in the system. The third component is a symmetric copolymer/surfactant $\mathrm{A}_{N} \mathrm{~B}_{N}$. Typical for microemulsions, we take the block size $N$ larger than the solvent size $n$. Our default system is chosen as $n=4$ and $N=20$ and we note that variations for these parameters will give only quantitative differences. The overall composition of the system is in the grand canonical ensemble sufficiently described by the surfactant/copolymer concentration $\varphi_{s}^{b}$. As there are just two types of segments, $\mathrm{A}$ and $\mathrm{B}$, there exists just one interaction parameter $\chi$. With the value of $\chi$ we not only tune the width of the solubility gap between the two solvents, but also modulate the affinity of the surfactant/copolymer for the A-B interfaces. 


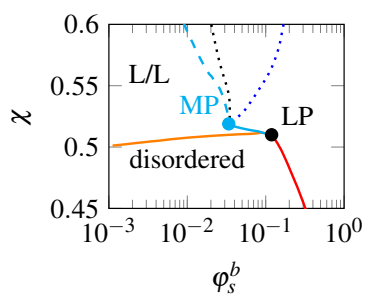

Figure 4.1: Phase diagram $\left(\chi, \varphi_{s}^{b}\right)$ for $(n=4$ and $N=20)$ in semi-logarithmic coordinates as found by SF-SCF theory. The MP (cyan dot) and the Lifshitz point (LP) (black dot) are indicated. The saturation line, which represents the microemulsification transition, is dashed (cyan). Dotted lines are Winsor III binodal lines. Continuous lines represent lines of critical points. The Leibler line is in red, the Scott line in orange, the line connecting the MP and the LP is in cyan. The disordered phase and liquid-liquid phase separation (L/L) regions are indicated. Regions for microphase segregation (to the right of the red line), and the microemulsion regions (between the dashed and the dotted lines) are not pointed at. (Further details are in the text. We will adhere to similar color coding for results to follow.

As it will become clear, this model produces rich results that are relevant for microemulsions. The same model has been used before by several authors [40, 109-111]. Earlier works explicitly focused on mapping out different regions of the phase diagram in $\chi$ and $\varphi_{b}^{s}$ coordinates $[112,113]$. It is useful to review what is already known. The phase diagram exemplified by Fig. 4.1 is not taken from the literature, but is generated by the present SF-SCF method using the default $n=4$ and $N=20$ parameters. The SF-SCF method basically reproduces the known aspects of this phase diagram accurately. In Fig. 4.1 we not only present the known regions and lines of this phase diagram, but we have added the newly obtained results that are relevant for microemulsions. In the following we will use this phase diagram to review the literature and preview our results.

In Fig. 4.1 we see several lines which demarcate various phases. Going clockwise, we have (i) a disordered region, (ii) a region where there is a liquid/liquid interface between two macroscopic phases (L/L), (iii) a one-phase microemulsion region (in between the dashed cyan and the dotted line), (iv) a three-phase microemulsion region (in between the dotted lines), and ( $\mathrm{v}$ ) a microphase segregated region at high copolymer concentrations (to the right of the dotted line all the way to the red line).

It is of interest to give some insight in how the demarcation lines were obtained. One limit of our model is the liquid-liquid interface $\left(\varphi_{s}^{b}=0\right)$. Knowledge about how 
liquid-liquid interfaces behave dates back to Van der Waals[43, 114]. The binary solvent has a solubility gap when $\chi>\chi^{b}=2 / n$. (In the default system we thus have $\left.\chi^{b}=0.5\right)$. This means that when $\chi<\chi^{b}$ the solvents mix homogeneously. When $\chi>\chi^{b}$ we have two macroscopic phases and the interface between these phases has a finite tension. The protocol to locate this critical point is simple: we start in the two-phase $(\mathrm{L} / \mathrm{L})$ region and then decrease the $\chi$ until the homogeneous phase is found. The surfactant-free limit is obviously outside the plotted range of Fig. 4.1.

One can next introduce copolymers/surfactants in such systems (while remaining balanced) and follow a similar protocol (that is, start at the $\mathrm{L} / \mathrm{L}$ state and reduce the $\chi$ ) to find $\chi^{b}$-like critical points. The Scott line connecting such points is shown in Fig. 4.1 in orange. It is seen that small amounts of surfactant typically increases the bulk critical point of the system. Scott line comes to an end at Scott point (SP). At SP the distance between the interfaces $D=\infty$.

When both solvents are removed and the surfactant/copolymer $A_{N} B_{N}$ has a concentration $\varphi_{s}^{b}=1$, we arrive at systems that are known to show microphase segregation. For symmetric copolymers we must be in a lamellar phase $L_{\alpha}$. Starting from this lamellar phase and decreasing the interaction parameter invariably will lead to the disordered state. We exit the microphase segregated region by construction at a critical point[115], which for the presented model occurs at $\chi^{m s}=10.495 /(2 N)=0.26$ (again outside the presented range). We can subsequently add equal amount of solvents (recall, we need to remain balanced), and follow the same protocol to obtain $\chi^{m s}$-like critical points. The Leibler line connecting such points is presented in Fig. 4.1 in red. It is necessary to mention that in the microphase segregated systems there is a repeat distance $D$. Upon addition of solvents this $D$ varies gradually. Correspondingly, along the Leibler line there exists also a characteristic distance $D$ reminiscent of the lamellar phase periodicity which gradually increases when $\varphi_{s}^{b}$ decreases. Thus the Leibler line comes to an end at the Leibler point (LBP) with a finite $D$. In the limit of infinitely long polymer chains the repeat distance $D \rightarrow \infty$.

It is well known that the Leibler line meets the Scott line at a tricritical point [116119] usually referred to as the Lifshitz point in the limit of infinitely long polymers. We will refer to this point as classical Lifshtiz point (CLP) in this work. However, for finite chain length the Scott line and Leibler line do not meet. In other words, the classical Lifshitz point does not exist and we will instead have two points: the end point of the scott line (SP) occuring at $D=\infty$ and the end point of the Leibler line (LBP) occuring at finite $D$. It is interesting to note that SP and LBP occur at the same $\left(\chi, \varphi_{s}^{b}\right)$ yet at different $D$. It is possible to obtain the Scott line for interfaces with a finite imposed distance. The modified protocol produces a 'constrained' Scott line. The end point of such line provides us with constrained Scott points (CSP). Collecting these points produces a line that connects the SP 
with LBP. Along this line only $D$ varies while $\chi, \varphi_{s}^{b}$ remains constant, and thus cannot be seen in Fig.1. The point where the CSPs meet LBP will be referred to as the Lifshitz point (LP) for real systems (for convenience) and can be seen as a black dot in Fig.1. LP occurs at $\chi^{l}=2\left(1+2(n / 2 N)^{2}\right) / n$ (in our case $\chi^{l}=0.51$ ). In Fig.1 SP, LBP, LP lie on the same spot.

The remainder of the phase diagram Fig.1 is relevant for microemulsions and therefore will be discussed at length later. In this phase diagram we plot lines of critical points as continuous lines and lines of first order phase transitions as dashed and dotted lines. Most of our results are of the latter type.

Just above the Scott line the system has two phases and there is a macroscopic interface with finite tension $(\mathrm{L} / \mathrm{L})$. Let's focus on the interfaces and consider what happens when we increase the $\chi$ at fixed $\varphi_{s}^{b}$ or similarly increase $\varphi_{s}^{b}$ at fixed $\chi$. In both modes we can enter the scenario that the interfacial tension of the interface starts to decrease. As the system is balanced this reduction can proceed until the tension vanishes. When we reach this tensionless state, we witness a microemulsification transition. Addition of even more surfactants/copolymer will be met by generating internal interfaces. Soon we enter the state that the amount of interface is proportional with the amount of surfactants added. At all these interfaces there exists a 'saturated' amount of surfactants/copolymers. It is argued below that this microemulsification transition is of the first-order type. It is proven that there are many intricacies associated to this phase transition. One of the peculiarities is the observation that for this phase transition there is no coexistence between 'phases' possible. The reason for this will be discussed. Collecting points $\chi-\varphi_{s}^{b}$ for which the interfacial tension vanishes leads to the so-called saturation line. The existence of this line is mentioned in the literature[120] but its location in the phase diagram is not well documented and it is often assumed to meet LP because of numerical difficulties. We found that the saturation line ends at a critical point. Because of its importance for microemulsions we will refer to this critical point by the microemulsification point (MP). The saturation line and its critical point are given in Fig.1 by a cyan dashed line and a dot, respectively. It is significant to mention that the saturation line does not meet the Scott line nor the Leibler line and thus the MP and the LP are different points.

In the procedure that gave us the saturation line and the corresponding MP, it was implicit that the interfaces that we considered were far apart, that is, the interfaces were non-interacting. However, it is possible to modify the protocol and consider explicitly interacting interfaces. By imposing a fixed distance between interfaces at fixed $\chi$ we can determine the polymer concentration that saturates the interfaces. This modified protocol produces 'constrained' saturation lines. The end-point of a constrained saturation line gives an MP-like critical point. We will refer to these points as 'constrained MPs' (CMP). Collecting these critical points produces a line in between the MP and the LP which is given in Fig.1 by the solid 
cyan line (as it is a line of MP-like critical points). Going along this line from the MP to the LP, the distance $D$ decreases.

We note, in $\left(\chi, \varphi_{s}^{b}, D\right)$ - coordinate there exists a region between MP, LP and $\mathrm{SP}$ which we can refer to as 'supercritical triangle.' Without the artificial connection between SP and LP, only one side of the triangle is closed (MP-LP) while leaving two open sides (SP-LP, SP-MP). Apparently, this allows to enter the disordered state from the ordered state without undergoing a phase transition. In the limit of infinitely long polymers, this supercritical triangle becomes smaller and vanishes into the classical Lifshitz point. For real systems, the classical Lifshitz point does not exist and there only exists a supercritical triangle. We will not explore the supercritical triangle in this work as it lies within the domain of simulations and will be published elsewhere.

In the microemulsion literature the transition from a single phase microemulsion to a three-phase microemulsion (Winsor III) is well known. In our simple model we find also evidence for such phase transition. This transition is more classical and the blue and black dotted lines in the phase diagram Fig.1 are the binodal lines connected to this phase transition. The binodal lines meet, as usual, at a critical point which happens to be the MP. To understand how this classical phase transition comes about, it is necessary to understand what causes the cohesiveness of the middle phase in the Winsor III microemulsion. It is shown that a nonmonotonic interaction between surfactant-loaded interfaces is fundamental to the cohesiveness. The interaction curves are shown to be the result of the sum of a power-law decay (steric repulsion between surfactant blocks) and an exponentially (overlap of tanh profile of $\mathrm{L} / \mathrm{L}$ interfaces) decaying contribution. As compared to the DLVO-potential the signs of the two contributions is reversed and therefore the interaction curves are said to be of the inverse DLVO type.

The interfaces that are relevant for microemulsions are tensionless. The physics of such interfaces is controlled by the bending rigidities. The mean bending modulus, which is positive, controls the shape fluctuations of the interfaces. The Gaussian bending modulus controls the topology of the interfaces. A sign switch of this parameter will also indicate a phase transition in the system. The phase boundaries for this type of transitions are not yet included in our phase diagram because to date we have insufficient information to precisely locate such bounds. In a recent paper we have shown how to evaluate the bending rigidities unambiguously for balanced tensionless interfaces. We have used this protocol to find the mechanical parameters for cases along the saturation line and show that these parameters vanish upon approaching the MP with a power-law coefficient of unity. The exponent has an unusual value which is attributed to the observation that at the critical point the interfacial width remains finite and the density difference does not vanish, that is, at the MP the interface does not vanish. Only internal interfaces disappear.

In the remainder of this chapter we will briefly outline the premises of the 
SF-SCF theory that is used. Above we have introduced the model that is used already and therefore the theory is not presented in its most general form but rather targeted to deal with this model. In the results we will discuss isolated interfaces in the first part. The interactions between interfaces is the topic of the second part. In a third and final part we will address how the mechanical parameters of the tensionless interfaces vary along the saturation line. These last results play a key role to unravel the topological structure of the microemulsion systems. In the discussion we will outline how our results match up with experimental information on microemulsions. Here we will also reflect on some generalisations of the models and how open issues might be worked on in the future. As usual we end with our conclusions.

\subsection{Scheutjens-Fleer self-consistent field theory}

At the basis of the self-consistent field (SCF) theory there is a mean field free energy for molecularly inhomogeneous systems. Scheutjens and Fleer use a discretisation scheme for the spatial coordinates $r$ as well as for the segments $s$ in the chains [53, $55-57,91-93]$. This free energy $F$ is thus given [53] in terms of discrete volume fraction distributions $\varphi_{x}(r)$ and complementary segment potential $u_{x}(r)$ profiles for segment types $x=A, B$. Lagrange parameters $\alpha(r)$ are introduced to implement the (local, i.e. per coordinate $r$ ) incompressibility of the system. The SF-SCF approach thus starts with the free energy functional

$$
\begin{array}{r}
F=-\ln Q([u])-\sum_{x, r} u_{x}(\mathbf{r}) \varphi_{x}(\mathbf{r}) L(r)+F^{i n t}([\varphi]) \\
+\sum_{\mathbf{r}} \alpha(\mathbf{r}) \cdot\left[\sum_{x} L(r) \varphi_{x}(\mathbf{r})-L(r)\right]
\end{array}
$$

In this equation we have the system partition function which can be decomposed into single chain partition functions $q_{i}$ for molecule component $i=A_{n}, B_{n}, A_{N} B_{N}$ : $Q=\Pi_{i} q_{i}^{n_{i}} / n_{i}$ !. The molecular partition function $q_{i}$ can be computed in freely jointed chain (FJC) approximation from the (known) segment potentials $\left[u_{x}(r)\right]$, and $n_{i}$ is the number of molecules of type $i$ in the system. We use the Bragg Williams approximation, similarly as in regular solution/Flory Huggins theory, to find the interaction free energy

$$
F^{i n t}=\chi \sum_{r} L(r) \varphi_{A}(r)\left[\varphi_{B}(r)+\frac{1}{6} \nabla^{2} \varphi_{B}(r)-\varphi_{B}^{b}\right],
$$

wherein $L(r)$ is the number of lattice sites at coordinate $r$. The super index $b$ refers to the quantity in the bulk solution (which exists far from the interface). SCF 
solutions now involve optimizing the free energy $(F)$ with respect to its variables, respectively segment potentials, volume fractions and Lagrange field.

Optimizing the $F$ with respect to the volume fractions, we obtain a way to evaluate the segment potentials as follows,

$$
\frac{\partial F}{\partial L(r) \varphi_{x}(\mathbf{r})}=0 ; \Longrightarrow u_{x}(\mathbf{r})=\alpha(\mathbf{r})+\frac{\partial F^{i n t}}{\partial L(r) \varphi_{x}(\mathbf{r})}
$$

Hence $u_{A}(\mathbf{r})=\alpha(\mathbf{r})+\chi\left(\varphi_{B}(\mathbf{r})-\varphi_{B}^{b}+\frac{1}{6} \nabla^{2} \varphi_{B}(r)\right)$. A similar equation is found for $u_{B}$.

Optimizing the free energy $(F)$ with respect to the segment potentials shows the way to evaluate the volume fractions as follows,

$$
\frac{\partial F}{\partial u_{x}(\mathbf{r})}=0 ; \Longrightarrow L(r) \varphi_{x}(\mathbf{r})=-\frac{\partial \ln Q}{\partial u_{x}(\mathbf{r})}
$$

The propagator formalism[57], which can be shown to do the same, is the preferred way to find these densities. Finally, $\partial F / \partial \alpha(\mathbf{r})=0$ says that the optimization should obey the compressibility relation $\sum_{x} \varphi_{x}(\mathbf{r})=1$.

Numerical solutions that obey these requirements have the property that the potentials both determine and follow from the volume fractions profiles and vice versa and are said to be self-consistent. Besides structure of the interfaces (density distributions) we can evaluate the thermodynamic quantities. Importantly there is a closed equation for the grand potential density

$$
\begin{array}{r}
\omega(r)=-\sum_{i} \frac{\varphi_{i}(\mathbf{r})-\varphi_{i}^{b}}{N_{i}}-\alpha(\mathbf{r})-\chi \omega^{i n t} \\
\text { where, } \omega^{i n t}=\varphi_{A}(\mathbf{r})\left[\varphi_{B}(\mathbf{r})+\frac{1}{6} \nabla^{2} \varphi_{B}(\mathbf{r})\right]-\varphi_{A}^{b} \varphi_{B}^{b}
\end{array}
$$

and the overall grand potential is found from $\Omega=\sum_{r} L(r) \omega(r)$. For detailed derivations, please refer to earlier works $[53,54,121-124]$. For the planar interface, the interfacial tension is found from $\gamma=\Omega / A=\sum_{z} \omega(z)$ with $A$ the area of the $\mathrm{L} / \mathrm{L}$ interface and $z$ the coordinate across the planar interface.

The chemical potentials follow from the bulk volume fractions (reference system), $\varphi_{i}^{b}$, where $i=$ oil, water, surfactant, and a generic equation for it is available from the Flory-Huggins theory:

$$
\begin{array}{r}
\mu_{i}=\ln \frac{\varphi_{i}^{b}}{N_{i}}-\frac{N_{i}}{2} \sum_{X} \sum_{Y}\left(\varphi_{X}^{b}-\frac{N_{i}^{X}}{N_{i}}\right) \chi_{X Y}\left(\varphi_{Y}^{b}-\frac{N_{i}^{Y}}{N_{i}}\right) \\
+1-N_{i} \sum_{k} \frac{\varphi_{k}^{b}}{N_{k}}
\end{array}
$$


wherein $N_{i}^{X}$ is the number of segments of type $X$ in molecule $i$.

The SF-SCF solutions are routinely generated using direct inversion in iterative subspace technique (DIIS) with a tolerance of $10^{-7}$ and when necessary (calculations near MP) the accuracy was improved to $10^{-10}$. We again refer to the literature for details on the numerical scheme that was used[58]. At the system boundaries there are mirror-like boundary conditions. The mean-field approximation is applied along layers of lattice sites (e.g. in $x$ and $y$ directions) and gradients (e.g. in segment densities) are evaluated in the remaining $(z)$ coordinate. Typically the planar coordinate system is used. Cylindrical or spherical coordinate systems were employed in the evaluation of the mechanical parameters of the interfaces.

\subsection{Results}

Our results will be discussed in three sections: (i) Isolated interfaces: We will initially consider interfaces that are far apart and do not interact. Primary focus is to understand the saturation line and to introduce the microemulsion point (MP). We will further discuss the order parameter and peculiarities associated with the phase transition that occurs at the saturation line. (ii) Interacting interfaces: We will show that the MP merges with Lifshitz point (LP) for real systems when a finite distance is imposed between the interfaces. We will discuss the appearance of the Winsor III phases with the middle phase featuring lamellae with finite repeat distance, as a metastable state. We will also analyze the interaction between saturated interfaces and prove that the cohesiveness of the middle phase occurs as a consequence of non-monotonic interactions. (iii) Mechanical properties: Finally, interfacial properties such as bending rigidities will be evaluated and discussed. Focus will be to understand their behavior along the saturation line.

\section{Isolated interfaces}

For our molecular model at a specified interaction parameter $\chi$ and bulk concentration of surfactant specified by $\varphi_{s}^{b}$, we briefly explained how relevant thermodynamical equilibrium properties can be obtained using SF-SCF theory. In the limit of no surfactant, when $\varphi_{s}^{b}=0$, we have a system of pure solvents $A_{n}, B_{n}$. From the phase diagram presented in Fig. 4.1, it can be easily observed that the solvents phase separate when $\chi>\chi^{b}$ and features a macroscopic L/L $\left(A_{n} / B_{n}\right)$ interface with finite interfacial tension $\gamma$. In our calculations, we prevent interaction between the interfaces by placing them far apart. Such interfaces are called isolated interfaces. The distance between the interfaces, called repeat distance, as introduced earlier remains infinitely large $D \rightarrow \infty$.

We can now introduce a finite amount of surfactant to this macroscopic L/L interface and consequently reduce equal amounts of solvent to remain in a balanced 


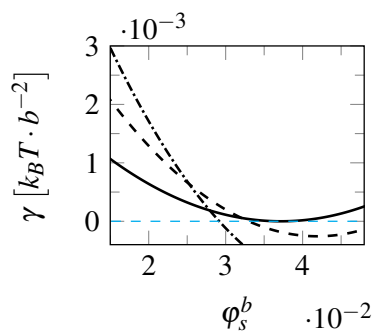

Figure 4.2: Interfacial tension, $\gamma$ [in units of $k_{B} T / b^{2}$ with $b$ a segment length] of the planar interface as a function of bulk surfactant concentration $\varphi_{s}^{b}$ in the system. $n=4, N=20$. Interaction parameters are: $\chi=0.52$ (solid), 0.53 (dashed) 0.54 (dash-dot). Blue dashed line represent $\gamma=0$.

state. Addition of surfactants to the $\mathrm{L} / \mathrm{L}$ interface can be visualized as increasing the $\varphi_{s}^{b}$ at a fixed $\chi$ in Fig. 4.1. It is thus possible to record the $\gamma$ as function of $\varphi_{s}^{b}$ at fixed $\chi$. In Fig. 4.2 we present such a graph for $\chi=0.54$ (solid), 0.53 (dashed), 0.52 (dash-dot).

As expected we observe a decreasing trend for $\gamma$ as a function of $\varphi_{s}^{b} \cdot \gamma$ is finite and positive at low values of $\varphi_{s}^{b}$ and gradually reaches zero and becomes negative at high values of $\varphi_{s}^{b}$ (e.g. for $\chi=0.54$ ). Negative values in $\gamma$ occur as a consequence of fixing the interfacial area in the mean field approach. For a fixed $\chi$, the tensionless state occurs at a specific bulk concentration of the surfactant $\varphi_{s}^{b *}$. By collecting such $\left(\chi, \varphi_{s}^{b *}\right)$ we obtained and presented the saturation line (cyan dashed line) in Fig. 4.1. Please refer to the supplemental information for equilibrium solutions (volume fraction profiles, grand potential density profiles).

To appreciate the fascinating physics of the saturation line, it is essential to elaborate on what happens to the system when we follow the previous protocol (i.e. increase the $\varphi_{s}^{b}$ at a fixed $\chi$ ). To reiterate, we start with a $2 \phi$ system (macroscopic $\mathrm{L} / \mathrm{L}$ interface). By gradually adding surfactants, we remove equal amount of solvents from the system. By composition and contents we have a balanced system with zero spontaneous curvature. This prevents formation of droplets of liquid in liquid when surfactant is added. When we have sufficient amount of surfactant, suddenly an enormous amount of 'internal' interfaces appear in the system as tension reaches zero. Thus at the saturation line, since there is no restriction to form additional interface, a phase transition from $2 \phi$ (macroscopic L/L interface) to $1 \phi$ (Winsor IV) occurs. We refer to this transition as 'microemulsification.' Several experiments report existense of bicontinuous microemulsion phases[102, 109, 125, 126] close to the presented saturation line. It can be observed that this trend does not continue and the saturation line comes to an end. This happens at a special point when 


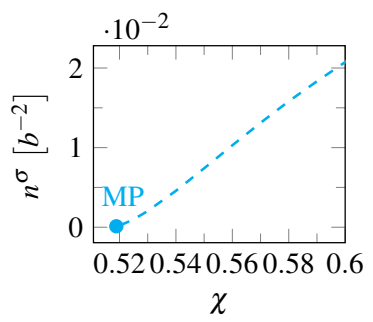

Figure 4.3: Adsorbed number of surfactants (per unit area) in the tensionless state $n^{\sigma}$ as a function of interaction parameter $\chi$. Inherently, the values are presented along the saturation line (see, Fig. 4.1) as it is the only line corresponding to tensionless state. End-point of the saturation line is indicated as MP, shown as cyan dot.

$\chi=\chi^{m}=0.518875$ for our model. We refer to this point as microemulsion point (MP). For $\chi>\chi^{m}$, there exists a $\varphi_{s}^{b}=\varphi_{s}^{b *}$ where microemulsification occurs, and for $\chi<\chi^{m}$, there exists no $\varphi_{s}^{b}$ for which microemulsifcation occurs. It should be noted, however, that we find macroscopic finite tension $\mathrm{L} / \mathrm{L}$ interfaces below MP until we reach the Scott line. This is a proof that MP does not end at the Scott line, confer Fig. 4.1.

When we define the number of surfactants per unit area of the internal interface $\left(n^{\sigma}\right)$, it can be clearly stated that $n^{\sigma}=0$ for finite tension L/L interface (as there is no internal interface). As soon as microemulsification occurs, we have a finite nonzero $n^{\sigma}$. As stated before we have a peculiar phase transition, the internal interface emerges and does not coexist with macroscopic L/L interface. This is evident also from Fig. 4.1. Apparently, there is just one saturation line where internal interfaces appear. A second binodal line does not exist, strongly indicating an emerging first-order phase transition. Such non-classical phase transitions are known to exist. Analogies can be found in the coil-flower transition in polymer brushes [67, 127-129] and the first appearance of surfactant micelles [130]. Microemulsification thus features a discontinuity in the first derivative of $\gamma$ with respect to $\mu$, which can be realized from Gibbs-Duhem equation: $\partial \gamma / \partial \mu=-n^{\sigma}$ (at micremulsification) and $\partial \gamma / \partial \mu=0$ (before microemulsification). By Ehrenfest classification, such phase transitions are clearly first-order[131]. While considering phase transitions, an appropriate order parameter should be zero before the transition and jump to a non-zero value after transition [132]. Conveniently, $n^{\sigma}$ turns out to be the relevant order parameter for microemulsification.

It is of interest to understand how the order parameter varies along the saturation line. In Fig. 4.3, we present $n^{\sigma}$ as a function of $\chi$. Sufficiently far from MP in the saturation line $\chi \gg \chi^{m}$, significantly high coverage of surfactants at the interface 
will be required to observe internal interfaces (microemulsification). In such scenario, $n^{\sigma} \rightarrow 1$ as seen in Fig. 4.3, and surfactants are densely packed closely at the interface closely resembling a double-sided polymer brush. In this limit we understand that the adsorption of the surfactant has a free energy barrier associated to it. A Surfactant chain first has to stretch and go through the brush before it can breach (and adsorb onto) the interface. This barrier makes the appearance of the internal interface jump-like. Very close to the MP however the coverage is low (cf. Fig. 4.3). In this case the adsorbed amphiphiles have a close to Gaussian conformation at the interface. We anticipate that adsorption of amphiphiles in this case can proceed without an adsorption barrier. As a result, the appearance of the internal interface in the near MP-region becomes a smooth process (that is, not jump-like). Yet, we find $n^{\sigma} \approx 1.1788 \times 10^{-4}$ (which is significantly above zero and does not vanish at MP) at the end point of saturation line (MP). We note that, the purpose of the presented work is to prove the existence of the MP and it's control over micremulsion behavior. We refrain from detailed analysis of the order parameter and the phase transition behavior. However, by providing a flavor we hope to provoke scientific curiosity towards such analysis.

Let's summarize the results presented in this section. We considered isolated interfaces. As expected addition of surfactants reduced $\gamma$ leading to a tensionless interface at high $\chi$. By collecting $\varphi_{s}^{b *}$ values for every $\chi$ we constructed a saturation line in Fig. 4.1. By virtue, at the saturation line, since there is no restriction to form additional interface, a phase transition from $2 \phi$ (macroscopic $\mathrm{L} / \mathrm{L}$ interface) to $1 \phi$ (Winsor IV) occurs. Such microemulsification was argued to be a non-classical first-order phase transition. The end point of the saturation line is identified as a special point for microemulsions (as microemulsification can not occur beyond this point) and hence we referred to it as microemulsion point (MP). Futhermore, a possible order parameter and pecularities of microemulsification were discussed. It can be seen from Fig. 4.1, that the MP is located above the Scott line (for which the imposed distance $D=\infty$ ). We discussed that at Leibler line and LP the $D$ is finite. From the above observations, it is evident that we have to consider the effect of imposed distance on the MP. This will possibly provide insights on several other features discussed in the phase diagram. Hence we consider interacting interfaces in the following section. In experiments this can be achieved by increasing the amount of surfactants. Since the interfacial area is proportional to the amount of surfactants, the interfaces grow and naturally come closer to each other. Briefly, isolated interfaces occur in dilute systems and the interfaces interact in highly concentrated systems. 


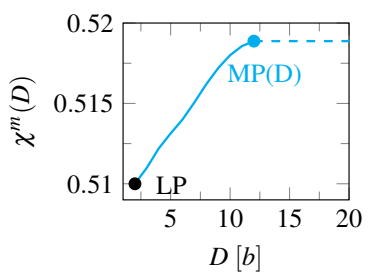

Figure 4.4: Interaction parameters $\chi^{m}(D)$ for MP-like critical points a function of the imposed distance $D[b]$. The line of such critical points ends at the Lifshitz point (LP) $\chi=0.51$. Dashed cyan line represents independence of MP for $D \approx 12$. All such points merge and lie at MP i.e. they occur at same $\varphi_{s}^{b *}$ in Fig. 4.1. Solid cyan line is equivalent to line of 'constrained MPs' presented in Fig. 4.1. Evidently, as $D$ decreases $\varphi_{s}^{b *}$ increases.

\section{Interacting interfaces}

To consider the effect of finite imposed distance $D$ on the end point of the saturation line, we perform a similar calculation as discussed in Section. III A., by imposing a finite distance $D$ between the interfaces. This can be easily achieved by reducing the system size $(M)$ in our calculations. As we employ reflecting boundary condition, the distance between the interfaces is given directly by the system size $(D=M)$. Note that we ensure equal amount of solvents as always to remain in the balanced state.

For each imposed $D$ we have a saturation line and associated to this an endpoint resembling MP, which is evaluated using the previous protocol. Since we impose a constraint on the distance between the interfaces we refer to the evaluated end-points as 'constrained microemulsion points' (CMP). We present CMP as a function of $D$ in Fig. 4.4. When $D$ is decreased from $\infty$ to $\approx N$ we do not observe any change in the value of $\chi^{m}$ where the saturation line ends (dashed cyan line). Beyond this, the end point becomes a function of imposed distance (solid cyan line) and meets the Lifshtiz point. Furthermore, going from the MP to the LP by reducing $D$ the surfactant concentration increases dramatically (from a very low value of $\varphi_{s}^{b} \approx 3.4 \times 10^{-2}$ at the MP to about $\varphi_{s}^{b} \approx 0.12$ at the LP). The increase in surfactant concentration along the constrained saturation line can be seen in Fig. 4.1. In the phase diagram, only the saturation line with $D=\infty$ is presented.

In microemulsion literature, a phase transition from Winsor IV to Winsor III is well known. Indeed, we observe a similar transition within our one-parameter model for microemulsions. Such a transition is observed when we increase the amount of copolymer in the system to significantly higher values (in practice we increased the surfactant amount step-wise using the SCF solution with lower amounts as an 


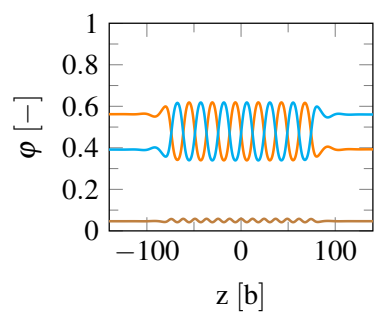

Figure 4.5: The volume fraction profiles $\varphi(z)$ of the two solvents $(n=4$, orange and cyan) and copolymer $(N=20$, brown curve) for $\chi=0.53$ for a system with a large amount of copolymer in the system $\theta=14.5$ for $M=300$ indicative of a Winsor III system with inter-lamellar spacing $D=13.5$ coexisting with two featureless solvent phases with a much lower copolymer content.

initial guess). Intuitively, one would expect to find multiple liquid-liquid interfaces homogeneously distributed throughout the system. However, as can be seen from Fig.5, we found instead an inhomogeneous distribution of molecules in the volume: two homogeneous solvent rich phases coexist with a phase characterised by many interfaces at regular spacings: in this case with an inter-lamellar spacing $D \approx 13.5$ (for $\chi=0.53, n=4, N=20$ ). This result is indicative of a three phase Winsor III system. By collecting the $\chi-\varphi_{s}^{b}$ values for this transition provides us with one binodal (black dotted) line in Fig. 4.1; the second binodal line (blue dotted) is estimated by calculating the average polymer concentration in the lamellar middle phase. Since, for any $D>N$ the binodal has to meet at MP and $D<N$ they meet at their corresponding CMP, we believe the expected parabolic shape of the binodals turns into a distorted cusp shape.

This result implies that there must be either a local minimum in the 'free energy landscape' which keeps the lamellae close to each other or there must be a local free energy barrier which prevents the interfaces from drifting apart. To find this out, the calculations on systems that encompass three phases are not ideal as these systems have a finite grand potential, that is, these systems are not per se free of tension, nor are the chemical potentials of the molecules the same as for the isolated tensionless interface. The solution to this problem is to focus computations on one particular interface that exists inside a lamellar phase ordering and investigate how this interface interacts with its neighbours.

To consider the interaction between the saturated interfaces, we start with an interface at fixed $\chi>\chi^{m}$ and $\varphi_{s}^{b}=\varphi_{s}^{b *}$ that is isolated. Now by decreasing the system size $M$ while imposing a reflecting boundary, we can 'simulate' the neighboring interface at distance $d=M$ (note $d$ is not same as imposing one finite distance $D$ per $\chi$ as above). As always, we ensure balanced state. As the system is 
balanced the chemical potentials of both solvents (liquids) are equal to each other at all times. Let us refer to this by $\mu_{l}$. The chemical potential of the surfactant $\mu_{s}$ has a different value of course. To understand whether the tensionless interfaces attract or repel each other, we may focus on an exchange chemical potential $\tilde{\mu} \equiv \mu_{s}-(2 N / n) \mu_{l}$, because when we insert a copolymer with $2 N$-segments into the system we have to take $2 N / n$ solvent molecules (equal amounts of both solvents) out of the system. We can do this for a given value of $d$ and compare this to the value at $d \rightarrow \infty$ and obtain a measure for the interaction free energy (per copolymer molecule):

$$
F^{i n t}(d)=\tilde{\mu}(d)-\tilde{\mu}(\infty)
$$

When it is harder to insert a copolymer into system at distance $d$ compared to the insertion when $d \rightarrow \infty$, that is when $F^{i n t}(d)>0$ the lamellae must be repulsive and vice versa. In Fig. 4.6(a) we present the interaction free energy per molecule $F^{\text {int }}(d)$ as function of distance between the interfaces $d$ for three interaction parameters whose distance from the MP is $\Delta \chi^{m}=\chi-\chi^{m}=0.03,10^{-3}, 10^{-4}$. It must be noted from the start that these interaction curves are truly of the mean field type, first of all because we completely ignore the undulation contribution between the interfaces, and secondly because we did not allow for topological changes of the interfaces; by way of the mean field approximation the interfaces have to remain parallel to each other (in other words we assume an $L_{\alpha}$-phase). Inspection of these interaction curves reveals that indeed the anticipated non-monotonic features are recovered. When coming from a large value of $d$ to smaller values, we first notice repulsion between the interfaces. But at a close proximity of the interface, rather suddenly a local minimum appears. In the examples given in Fig. 4.6(a) all minima that are shown have a positive value, implying that they are local minima. Hence, these may classify as a meta-stable minimum. The global minimum still is given by the infinite separation. However, the appearance of the maximum which separates the local minimum for the global one is consistent with the Winsor III result discussed above (cf. Fig. 4.5). As can be seen the position of the minimum $D \equiv d_{\text {min }}$ increases when $\chi$ decreases. Hence the prediction is that the spacing in the lamellar phase decreases when the system goes to stronger segregation. Further, the depth of the minimum becomes closer to zero when $\chi$ decreases. Below we will discuss how the local minimum can indeed become the global minimum, but on the mean field level we did not find such result. Before we can elaborate on this result we need to understand why such complex interaction curves develop in the first place.

In Fig. 4.6(b) we have plotted the optimal spacing $D$, that is the distance $d$ for which the interaction curve has the local minimum value, as a function of distance from the MP $\Delta \chi^{m}$. But now we approach the MP along the blue dotted line (second binodal line of Winsor III phase) presented in Fig. 4.1. As noted already $D$ decreases when the system goes to stronger segregation and this trend is clear from both Fig. 6(a) and Fig. 6(b). Inversely, the spacing increases when going in the 


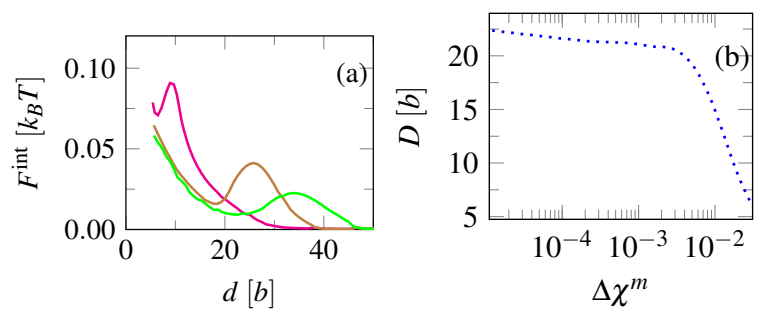

Figure 4.6: a) Free energy of interaction $F^{\text {int }}(d)$ [units $k_{B} T$ per sufactant] (see text) as a function distance between the interfaces $d$ for three values of the interaction parameter [ $\Delta \chi^{m}=0.03$ (magenta); $\Delta \chi^{m}=10^{-3}$ (brown); $\Delta \chi^{m}=10^{-4}$ (green); ]. Three point moving average values are presented to obtain a statistically accurate representation. (b) The optimal distance between the lamellae $D$ as a function $\Delta \chi^{m}$.

direction of weak segregation. However this increase in the spacing saturates. We have shown that the optimal spacing strongly correlates to the interfacial width $W$ (See section 5. of supplemental information).

We will briefly explain the origin of non-monotonicity in the interaction curves (For more details, see section 5. of supplemental information). Our conjecture to explain the non-monotonic interaction curves is the following. We know that two bare $\mathrm{L} / \mathrm{L}$ interfaces in close proximity attract each other: from the Van der Waals theory we know that the interfacial profile has a $\tanh (z / \xi)$ form. When two of such interfaces touch each other we find attractive forces. The length scale for this (exponentially decreasing) attractive interaction is the bulk correlation length $\xi$ or equivalently the width of the interface $W$. Of course, here we do not have bare interfaces: the copolymers have adsorbed onto these interfaces such that the interface becomes free of tension. These copolymers form a (weak) two-sided molecular brush. Forcing two such interfaces to interact will imply an overlap of such brushes. This brush-overlap gives a power-law like steric repulsion. Apparently this repulsion dominates both at small $d$ as well as at large $d$, but the attraction (reminiscent of the bare interfaces) is significant in between these limits. In such scenario, the position of the minimum is indeed expected to be proportional to $W$ (See Section. 5 of supplemental information, for more details). We will show in Section. III C that $W$ saturates upon approaching MP reasoning the saturation of $D$ observed. We note that the computations to establish the best $D$ for given $\chi$ are challenging and prone to numerical uncertainties. The leveling off at low $\chi$-values is however clear.

This non-monotonic overall interaction curve reminds us of the DLVO potential in charged colloids[133], which results from the combination of an attractive power- 
law dependent Van der Waals contribution and an exponentially decaying repulsive contribution due to the overlap of ion clouds. It is known that the local maximum in the DLVO potential exists at an inter-particle distance given by (twice) the Debye length (the length scale that characterizes the repulsion). With respect to the DLVO potential the signs have been reversed both for the power-law as well as for the exponentially decaying contributions. We may therefor refer to the non-monotonic interaction curve of Fig. 4.6(a) as an inverse DLVO potential.

There are several predictions we can make based upon the shape of the inverse DLVO potential. (i) When we insist on curves that have a local minimum (as in the current mean field calculations), we can only expect Winsor IV systems (homogeneous microemulsions). With an increase of the amount of surfactant in the system, the interfaces extend and the average distance between interfaces naturally must go down. At some threshold surfactant concentration we expect a phase transition in such Winsor IV system because at intermediate distances between interfaces, that is, when $d$ is near the maximum of the interaction curves, the system is unstable and must splits into a phase with short spacing between the interfaces and one with larger spacing between the interfaces. The energy barrier in the reversed DLVO potential prevents the lamellae to distribute homogeneously through the solution. (ii) In retrospect the result of Fig. 4.6 must be classified as a meta-stable result. It is caused by some initial guess which localized the interfaces in the middle of the system. The swelling of the middle phase, such that the lamellae spread over the volume, is prevented by the energy barriers. (iii) We understand that in the proximity of the SFP the amount of surfactants at the L/L interface can indeed be rather low. This means that the strength of the repulsion diminishes and the attraction becomes progressively more important. This causes the minimum of the inverse DLVO potential to increase in depth. (iv) If we could have allowed for a topological transitions of the middle phase, we could easily imagine that the local minimum becomes the global one. Then the result is truly consistent with the Winsor III systems.

To understand the topological behavior of such systems we need to consider the mechanical properties along the saturation line. In the following section, we will elaborate on the evaluation of such properties and discuss their behavior. Based on the results, we will discuss the expected topologies in Winsor IV region of the phase diagram.

\section{Mechanical properties (along saturation line)}

For microemulsions that have zero-tension interfaces, the bending rigidities are of central importance. In our earlier work[64] we reported an elegant route to unambiguously find the bending rigidities that feature in the Helfrich equation. Fundamental to this procedure is the use of the tensionless planar interface as the 
reference point for curving the interfaces. Next, we exploited the scale invariant properties of the Helfrich equation: when the curvature energy does not depend on the scale, it must be true that the chemical potential of the molecules in the system also do not depend on the scale. When the chemical potentials are fixed (do not depend on the curvature of the interface) one can identify the interfacial tension as the characteristic function. Then the Helfrich equation, a Taylor series expansion of characteristic function around mean $(J)$ and Gaussian $(K)$ curvature, reads

$$
\gamma(J, K)=\frac{1}{2} \frac{\partial^{2} \gamma}{\partial J^{2}} J^{2}+\frac{\partial \gamma}{\partial K} K \equiv \frac{1}{2} \kappa J^{2}+\bar{\kappa} K
$$

where $\kappa$ is the mean bending rigidity and $\bar{\kappa}$ is the Gaussian bending rigidity.

Three geometries were found for which the scale in-variance principle could be applied: (i) The first case is the spherical droplet with inner phase $\mathrm{A}_{n^{-}}$and outer phase the $\mathrm{B}_{n}$ rich phase. We add the surfactant until the Laplace pressure vanishes. The total curvature energy is now given by $\Omega=4 \pi R^{2} \gamma(J, K)$. As the mean curvature in this case is $J=2 / R$ and the Gaussian curvature $K=1 / R^{2}$ we find that $\Omega=4 \pi(2 \kappa+\bar{\kappa})$. Note that this result does not depend on $R$ (the hallmark for scale in-variance).

(ii) The second system is a case for which everywhere in the curved interface the mean curvature is zero. This occurs in a triple periodic bicontinuous $\operatorname{Im} 3 \mathrm{~m}$ cubic phase. The interface splits a unit cell exactly into two equivalent volumes, one filled by the A- and the other by the B-rich phase. We add surfactants until the chemical potentials are equal to the ones found for the planar tensionless interface. The curvature energy per unit cell is now only a function of the genus $g$ [95], $\Omega=4 \pi(1-g) \bar{\kappa}$. For the Im3m phase we have $g=3$ per unit cell. Again, the size of the unit cell is not involved in this result and the curvature energy is scale invariant (See section 3. supplemental information for computational details).

(iii) Interestingly there exists yet another system for which we can use scale in-variance and this is for the minimal torus. A minimal torus is a torus for which the major radius $R_{1}$ and the inner radius $R_{2}$ have a fixed ratio $R_{1} / R_{2}=\sqrt{2}$. [97, 98] Again we fill the torus inside with the A-rich phase while the B-rich phase is the exterior phase. As before we add surfactants until the Laplace pressure vanishes (cf. case i). The torus is an object with genus $g=1$ and thus has no contribution to the Gaussian curvature. The curvature energy for a minimal torus is given by $\Omega=4 \pi^{2} \kappa$.

All these cases can computationally be realised within the SCF framework and the results of this protocol gives consistent results for the bending rigidities[64]. Moreover we have shown that the Gaussian bending modulus follows from the second moment over the grand potential density of the tensionless planar interface.

In Fig. 4.7 we present results for both moduli as a function of $\Delta \chi^{m} \equiv \chi-\chi^{m}$ upon approaching MP along the saturation line. Inherently, we consider isolated 


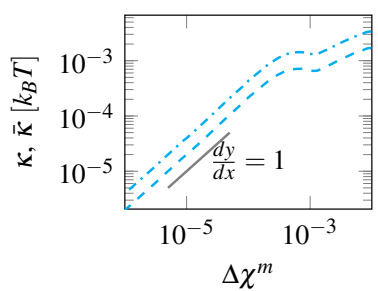

Figure 4.7: Critical scaling near MP. The mean $\kappa$ (dash dotted) and Gaussian bending modulus $\bar{\kappa}$ (solid) in unit of $k_{B} T$ as a function of $\chi-\chi^{m}$ in log-log coordinates. (Here $n=4$ and $N=20$ for which $\chi^{m}=0.518875$ ). Relevant slopes are indicated. Slightly outside the plotting range, namely at $\chi \approx 0.5415$, the $\bar{\kappa}$ switches sign; $\kappa$ remains positive for all $\chi$-values.

interfaces. We find, near the MP, both $\bar{\kappa}$ and $\kappa$ are positive, see Fig. 4.7. We note, that evaluation of rigidities for interacting interfaces remains unsolved and will be one of our future research interests. Furthermore, two regimes with different scaling can be clearly identified. Going from high to low $\chi$, between $10^{-3}<\Delta \chi^{m}<10^{-2}$ both $\bar{\kappa}$ as well as $\kappa$ decreases with a scaling exponent $1 / 2$. Beyond that, $\Delta \chi^{m}<10^{-3}$ the rigidites vanish with a scaling exponent of unity $\bar{\kappa} \sim \kappa \propto\left(\chi-\chi^{m}\right)^{1}$. Consistently, the ratio $\kappa / \bar{\kappa} \approx 2$. This ratio changes dramatically (not shown) when $\bar{\kappa}$ switches sign at higher $\chi$ than used in Fig. 4.7. Before we elaborate on the scaling exponents, it is vital to understand the behavior of interfacial width and area expansion modulus.
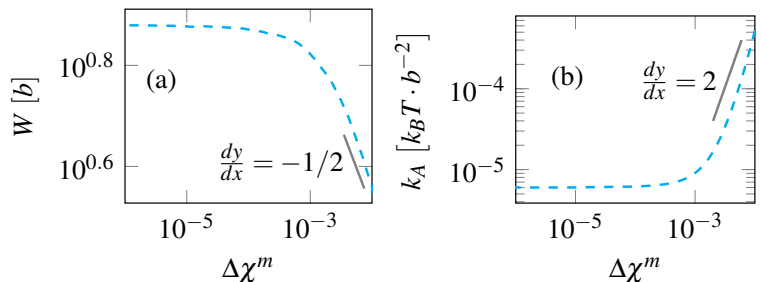

Figure 4.8: (a) Interfacial width $W$ of the interface in units $b$, as a function of $\chi-\chi^{m}$ in log-log coordinates. (Here $n=4$ and $N=20$ for which $\chi^{m}=0.518875$ ). The width is found from the planar interface as $W=\mid\left(\varphi_{A_{n}}^{b}-\varphi_{B_{n}}^{b}\right) /\left(\varphi_{A_{n}}\left(z_{0}-\right.\right.$ $\left.1 / 2)-\varphi_{A_{n}}\left(z_{0}+1 / 2\right)\right) \mid$. (b) Area expansion modulus $K_{A}$ as a function of $\Delta \chi^{m}$ in $\log$-log coordinates. Relevant slopes are indicated.

Earlier we showed that the distance between the lamellae of interacting interfaces saturates at $\Delta \chi^{m}=10^{-3}$ (see Fig. 4.6(b). Similarly, both the rigidities switch their scaling dependence at $\Delta \chi^{m}=10^{-3}$. As expected, both these phenomena 
are intricately linked to the behavior of the interfacial width $W$, see Fig. 4.8(a). Again, going from high to low $\chi$ in the region $10^{-3}<\Delta \chi^{m}<10^{-2}$, interfacial width increases $W \propto\left(\Delta \chi^{m}\right)^{-1 / 2}$ and saturates beyond that. Fascinatingly $W \approx N$ after level off, and it should be noted that such levelling off is directly controlled by block length of the surfactant. To add to this we present results for the area expansion modulus $k_{A} \equiv \partial \gamma / \partial \log A$ in Fig. 4.8(b) as a function of $\Delta \chi^{m}$ in double logarithmic coordinates. $k_{A}$ shows scaling behavior (as expected) in the $\Delta \chi$ region where the width of the interface is following its scaling dependence. In this regime the (mean field) slope of 2 is found. Near the MP, however, $k_{A}$ goes to a constant. A clear consequence of this is that at the MP the interfacial area fluctuations will remain finite, that is, these will not diverge.

We may elaborate on the values of the mentioned scaling exponents. At relatively high $\chi$-values we found both $\kappa \propto(\Delta \chi)^{1 / 2}$ as well as $\bar{\kappa} \propto(\Delta \chi)^{1 / 2}$. This $1 / 2$ coefficient is not uncommon in the strong segregation limit. For example, the tension between two polymer melts is known to depend on the square root of $\chi$. In a recent publication we have shown that for bare $\mathrm{L} / \mathrm{L}$ interfaces at sufficiently strong segregation both bending rigidities also were shown to follow the $1 / 2$ scaling [134].

Near the MP the scaling exponent of unity was obtained for both rigidities. This is a non-classical exponents as it deviates, for example, from the Van der Waals $3 / 2$ scaling exponent found for the way the interfacial tension vanishes upon the approach to $\chi^{b}$ in pure solvent systems. Of course upon approaching the MP the interfacial width does not diverge and the density difference between the bulk phases does not vanish and therefore the nature of the MP significantly deviates from a bulk critical point of pure solvent system. Arguably the non-classical exponent of unity must therefore be attributed to these deviations.

Interestingly, a scaling exponent of $5 / 4$ is found near the classical Lifshitz tricritical point $[31,40]$. This is not surprising, as from Fig. 4.1 LP connects with MP and the bulk critical point of pure solvent, one can expect the scaling exponent to be an average of the scaling exponents in the other two regimes $\left(\frac{1+3 / 2}{2}=5 / 4\right)$. Further research in this direction is required and we will not elaborate further as it is outside the scope of the present work.

Finally, let's try to understand how the MP is (numerically) placed in relation to the bulk critical point of the binary solvent system. It is anticipated that the closeness of the MP to the bulk critical point is a function of the block length of the surfactant. Motivated by this we present in Fig. 4.9, the difference of the MP and the bulk critical point, $\Delta \chi^{m-b} \equiv \chi^{m}-\chi^{b}=\chi^{m}-2 / n$, as a function of the length $N$ of the block of the surfactant in $\log -\log$ coordinates. Results for $n=2,4,8$ are shown. For all the solvent sizes we find that $\Delta \chi^{m-b} \propto N^{-2}$ to a good approximation. We know from Van der Waals theory (see above) that the width $W$ of the $\mathrm{A}_{n} / \mathrm{B}_{n}$ interface is given by $W \propto\left(\chi-\chi^{b}\right)^{-1 / 2}$ and that the numerical coefficient is a weak 


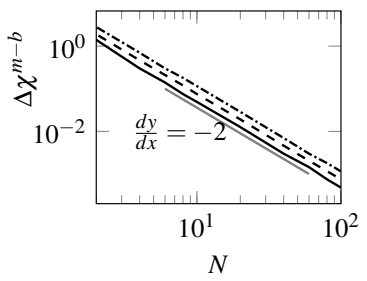

Figure 4.9: Behavior of MP in response to change in surfactant size. The difference between the MP and the bulk critical point, $\Delta \chi^{m-b}$ as a function of the surfactant block length, $N$ in double logarithmic coordinates. Lines are for: $n=2$ (solid), 4 (dashed), 8 (dash-dotted). The slope is indicated by a grey line.

function of $n$. Inserting $\chi=\chi^{s}$, we have $\Delta \chi^{m-b}=W^{-2}$. Clearly when $W \propto N$, we arrive at $\Delta \chi^{m-b} \propto N^{-2}$, which is the result of Fig. 4.9. The numerical coefficient is a small function of $n$, which can be traced to the $n$-dependent coefficient for the width of the bare interface. We conclude that MP is triggered by the condition that the width of the interface is proportional to the surfactant size.

\subsection{Discussion}

The idea that the tensionless state of the balanced L/L interface is of key importance to microemulsions is widely recognized[84, 135-137]. To our surprise, the idea that the loss of the tensionless state represents a true critical point with accompanied scaling behavior for the rigidities remained unexplored. In the context of weak adsorption of surfactant at the oil-water interface Safran discussed [135] a critical point using an analogy with the critical volume fraction of surfactants above which micelles form, in this case, above which interfaces can develop. To us this analogy is ambiguous because this might apply to all points on the line in our phase diagram (cf. Fig. 4.1) and not only to $\chi=\chi^{m}$. Safran argued that the interfacial rigidities have a finite value only above this critical surfactant concentration [135]. This result matches our prediction only at the MP, but it is in conflict with it for all other values of the interaction parameter. Indeed we argue that the control parameter is not the surfactant concentration alone in the system, but rather an 'effective' strength of adsorption/interaction, embodied in $\chi$, also features as another natural control parameter to reach microemulsion criticality. Furthermore, when imposed distance $D$ is considered as a third parameter, we observe that the classical Lifshitz point ceases to exist for real systems with finite $\mathrm{N}$ giving up to a critical region in $\left(\chi, \varphi_{s}^{b}, D\right)$-space. Neither the idea that an MP condition naturally develops near the Lifshitz point, nor the role of the surfactant length that determines $\Delta \chi^{m}$ (a 
result that is linked to our simple model with only one interaction parameter), nor the critical scaling of the rigidities, nor the (generic) sign switch of $\bar{\kappa}(\chi)$ at stronger segregation, were elaborated before. Finally, the inverse DLVO-potential which explains the cohesiveness of the middle phase in Winsor III is unexplored. All these aspects are closely linked to the MP critical point. We are only at the beginning to appreciate the impact of an MP for microemulsions.

Typically in microemulsion systems very short (low molecular weight) surfactants are used to generate bicontinuous emulsions [78, 80]. From the above it is clear why this is a good idea. For such a system the MP occurs far from the bulk critical point (cf Fig. 4.9) and hence one can relatively easily observe microemulsion sponge phases. In practice this means that it is not necessary to add huge amounts of co-solvents to reduce the difference in polarity between the two bulk phases (or increase $T$ ) and still come in the critical zone (near MP) where a sponge phase is possible. This trend was noticed before $[78,80]$, however, we feel that our interpretation is more physical than the rational presented earlier.

In addition, a Winsor III microemulsion can be obtained by an inverse DLVO potential. To have a local minimum and a maximum is only possible when the attractive contribution is of the same magnitude and range to that of the repulsive contribution. For short amphiphiles which only weakly adsorb on to the L/L interface, this is more easily realized than for longer amphiphiles [138].

Irrespective of the surfactant size, we found the following (generic) scenario for the rigidities: (i) Far from the bulk critical point $\left(\chi^{b}\right)$ and also far from the MP $\left(\chi^{m}\right), \kappa$ is high and positive and $\bar{\kappa}$ is negative. As argued earlier this implies that the lamellar phase is the expected topology. Following the classical way to understand the force balance in the lamellar phase we expect that repulsive undulation forces (increasing with decreasing $\kappa$ ) versus attractive Van der Waals forces will set the capabilities of this lamellar phase to swell (pick up water and/or oil). (ii) Upon approaching the MP along the saturation line (this can be done by adding cosolvents, or by increasing the temperature -implying a decrease in $\chi$-), the value of $\kappa$ decreases, but remains positive. The lamellar phase is expected to swell due to the increased Helfrich repulsion. (Other reasons why the lamellar phase swells when the system is closer to the MP are discussed below.) At some point the $\bar{\kappa}$ switches sign and becomes positive. From hereon we might expect a sponge-like phase to occur wherein the oil and water rich regions connect trough space (bicontinuous). Classically, the persistence length $l_{p} \propto \exp \left(\kappa / k_{B} T\right)$ sets the length scale of this sponge phase $[73,139]$. As $\kappa$ can become very small, one typically expects that $l_{p}$ becomes on the order of the molecule size. In this picture the persistence length in the middle phase decreases when the system goes towards MP.

The one-interaction parameter model is sufficiently rich to predict the appearance of a Winsor III system. (For symmetry reasons a transition to a two phase system -Winsor I or II- will not happen in a balanced model). In particular we expect the 
Winsor III phase to have a sponge-like middle phase, and this phase might expel both oil and water phases such that the interfaces in the middle phase become optimally spaced. This latter prediction is based on the interaction curve between (planar) saturated interfaces which was shown to be of the inverse DLVO type; an exponentially decaying attractive contribution is combined with a power-law repulsive contribution. The first is attributed to the overlap of two hyperbolic tangential profiles (bare interfaces) and the second due to the steric repulsion caused by the overlap of surfactants. In this setting the characteristic distance between interfaces in the middle phase is given by the width of the interface which (upon approach towards the MP) first grows but then saturated and is approximately constant in the vicinity of the MP. Combining this predictions with the Van der Waals forces and/or undulation forces (mentioned in the previous paragraph) leads to the prediction that there can be a non-monotonic dependence in the characteristic distance in the middle phase, while a weak trend to densify the middle phase is expected upon a final approach towards the MP. The appearance of the middle phase in a Winsor III in the close proximity of the MP is instrumental in the sense that the truly exotic properties of isolated interfaces near the MP is prevented from taking place. The fact that interfaces strongly interact in a Winsor III middle phase will in turn influence the values of both rigidities in yet unknown ways. We note that, the previous studies have proven that the effect of fluctuations causes long-range effective repulsion, which may stabilize the $1 \phi$ microemulsion near the MP[140-143], if $\bar{\kappa}<0$ for interacting monolayers. Analyzing the behavior of bending rigidities of interacting interfaces is beyond the scope of current work.

The mentioned theoretical trends correlate very well with known experimental observations. It is instructive to mention a few of these. (i) It is well known that for weak surfactants (short non-ionics) the one-phase microemulsion system is typically bicontinuous, that is, sponge-like [78]. This result is understood from the sign switch of $\bar{\kappa}$ that occurs for short surfactants for $\chi$ far from MP. (ii) Upon decreasing $\chi$ the microemulsion phase shifts to higher surfactant concentrations [144-146]. As mentioned already this result is caused by a decrease of interface persistence length upon approach to the MP and supported by the idea that the width of the interface -typical distance between interfaces on the mean field levelhas levelled off. (iii) For stronger surfactants (longer non-ionics), the lamellar phase $L_{\alpha}$ gradually becomes more pronounced. $L_{\alpha}$ first appears at low temperatures and high surfactant concentrations. At some higher temperature the lamellar phase swells and takes up most of the microemulsion phase volume, leaving only room for the sponge phase at rather thin slab in the microemulsion phase volume, namely at relatively low surfactant concentrations and relatively high temperatures [2]. This result is understood from the scaling dependencies of the rigidities. The larger (stronger) surfactants have (for given $T$ ) lower (or more negative) values for $\bar{\kappa}$, while shorter (weaker) surfactants are, for given $T$, closer to the MP and therefore are 
more likely to support the sponge phase [64].

The properties that we have discussed for the MP have many similarities with properties reported for the classical Lifshitz point (CLP). We must stress once again that the MP is not the same as the CLP. A classical Lifshitz point only occurs in the limit of infinitely long polymer chains. Furthermore, we identify that the Leibler line meets the constrained Scott line and refer to it as a Lifshitz point (LP) for real systems. We stress that such constrained Scott line is artificial and cannot be realized in experiments. In the limit of infinitely long polymers MP, LP, SP merges into a point creating the classical Lifshitz point (CLP), reasoning the similarities between the observed MP and reported CLP. However, CLP do not occur for real systems and is thus meaningless. For real systems, our results strongly suggest the existence of a supercritical triangle connecting the regions between LP, SP, MP in $\left(\chi, \varphi_{s}^{b}, D\right)$-coordinates. Future works may elucidate the fascinating physics in this region.

Microemulsions have a spectacular phase behavior. As we know this already for many years, we've got used to the extraordinary properties of these systems. We can mix oil and water (very complementary species) upto the nanometer scale and still 'walk' through the system in any direction without leaving the oil or the water phase (that is what the bicontinuous sponge phase allows us to do). The very close proximity of water and oil phases gives us the opportunity to make use of the complementary solvency conditions. There are already many applications of microemulsion that effectively make use of these amazing properties. This chapter only offers an explanation of why this happens.

The one-interaction parameter model that is used in this chapter is easily adopted to model correspondingly interesting systems. It is possible to deviate from the balanced case e.g. by selecting an asymmetric solvent $A_{N} B_{M}$ with $N \neq M$ or by taking different solvents, that is using a $A_{n}-B_{m}$ pair with $n \neq m$. In this case the microemulsification is first-order like as explained above. The corresponding critical point may also formally loose its strict meaning, but depending on the value of the spontaneous curvature, some of the physics of the corresponding 'criticality' may survive. Another interesting avenue to change the model is to remove one of the solvents. Then we have a block copolymer in a selective solvent which represents a toy-model for the lipid bilayer membrane [88, 147]. It is of interest to know whether in such a system there is an analogy with the MP. Finally, when both solvents are removed we arrive at the classical model for microphase segregation in block-copolymer systems [148, 149]. Again it is interesting to know the critical point in this system, found for $\chi N=10.5$ (here $N$ is the length of the copolymer), is connected to the current MP by a variation of the total amounts of solvents in the system. Such modifications of the one-interaction parameter model may unify the physics of seemingly remote fields of science; one may carry over, e.g., insights obtained for microemulsions into the microphase segregation systems and vice versa. 
Work in this direction is underway.

\subsection{Summary}

We have elaborated on a one-interaction parameter model for microemulsions and analysed it with the Scheutjens-Fleer self-consistent field theory. We focused on the weak segregation region and insisted on using a balanced molecular composition. Relevant for microemulsions we have identified a truly non-classical critical point, named microemulsion point (MP), which is linked to the ability of balanced L/L interfaces to become free of tension upon adsorption of surfactants. When the width of the interface exceeds the size of the surfactant the system cannot find the tensionless state and microemulsions cease to exist. MP controls the phenomenology of microemulsion systems which excellently correlates with known microemulsion behavior [73, 78, 144]. The following generic properties are predicted: (i) sufficiently far from the MP the $\bar{\kappa}$ is negative and a lamellar microemulsion is expected. (ii) However, near the MP the sign of the $\bar{\kappa}$ changes. This means that we expect the sponge-like topology of the system. (iii) Interestingly, when the $\bar{\kappa}$ and the $\kappa$ are of the same magnitude, the two moduli enter a scaling regime and it is predicted that they vanish at the MP (with scaling exponent unity). How much of this latter is relevant for applications is not yet known because close enough to the MP we expect that a Winsor III phase will form. This is concluded from an inverse DLVO-like interaction potential between surfactant loaded tensionless $\mathrm{L} / \mathrm{L}$ interfaces. The inverse DLVO potential explains why there is a particular length scale in the middle phase. The true structure of the middle phase, however, cannot be modelled by a one-gradient SCF model and therefore also the length scale in the middle phase may be subsequently influenced by undulations and Van der Waals forces. Thermal undulations can counter-intuitively stabilize lamellar topology by inducing longrange repulsion. We note, the competition bewteen repulsive thermal undulations, steric repulsion from finite amount of adsorbed surfactants, and attractive Van der Waals interaction determines the topology of middle phase. More elaborate (three-dimensional) simulations are needed to understand the intricacies of this middle phase and we have to wait for these results to correlate structure to e.g. known scattering data. 


\section{Appendix}

In the main manuscript, we introduced our model of ternary system. By maintining equal amount of solvents and using a symmetric surfactant, we achieve a balanced state (zero spontaneous curvature). For this model, we estimated the phase diagram and exposed the existence of a special critical point for microemulsion occurring at the end point of the saturation line. We referred to this point as microemulsion point (MP). In the supplementary information, we will provide additional details about several definitions used in the main text. In the first part, we will introduce how we define an interface mathematically. Later, we will elaborate on volume fraction profiles upon approaching the MP. We will show that interface widens, which can be quantified by defining interfacial width. Interesting evolution of pressure profiles will be discussed and related to bending rigidities. At the end of first section, we will present the technical details relevant to estimate the bending rigidities. In the second section, our primary focus is to discuss the origin of non-monotonic interaction observed in our system. Interaction between surfactant loaded liquid liquid interface can be roughly considered to be addition of interaction between liquid-liquid interface and interaction between end-tethered polymer brushes. We analyze such interaction and show the origin of non-monotonicity.

\section{Definitions and computational details}

\section{Mathematical definition of the interface}

To quantify the physics at the interfaces, we mathematically define the interface as an infinitely thin surface. Surfaces can be described in implicit form as $F(x, y, z)=0$. For such an implicit form one can obtain the normal to the surface by realizing that total derivative of $F$ vanishes: $d F=d r . \nabla F=0$, where $d r$ is a vector connecting two points on the surface. As $d r$ is a tangential vector, $\nabla F$ must be an orthogonal vector and points in the direction of normal to the surface. Thus, unit normal to the surface can be given by

$$
\hat{n}=\nabla F /|\nabla F|
$$

Reimann defined curvature of the surface as the change in the normal vector as one moves along the surface. Such a change is given by the curvature tensor $Q$. If we move along the surface by a distance $d r$, the normal $\hat{n}$ changes by an amount $d \hat{n}=d r \cdot Q$ where elements of tensor $Q$ is obtained by differentiating equation 4.9 . 


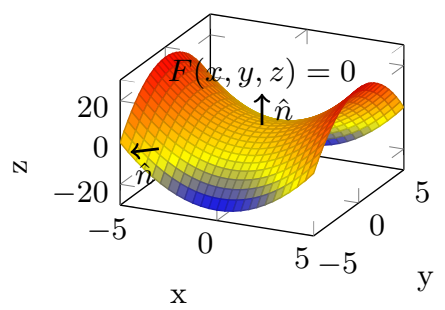

Figure 4.10: Illustration of mathematically defined interface $F(x, y, z)=0$, with normal vectors $\hat{n}$ shown for two sample points.

Curvature tensor $Q_{i j}$ is thus given by,

$$
Q_{i j}=\frac{1}{|\nabla F|}\left[F_{i j}-\frac{F_{i}|\nabla F|_{j}}{|\nabla F|}\right]
$$

where $F_{i}=\partial F / \partial r_{i}$. Such three dimensional tensors have three invariants under similarity transformations: trace, sum of principal minors and determinant. We know from explicit calculations that one of the eigen values is zero and thus the determinant. Thus, curvature tensor is a singular matrix. Two of the eigen values of $Q$ have dimensions of inverse length and are defined as the principal curvatures, namely $1 / R_{1}, 1 / R_{2}$. This allows us to define the invariant mean curvature as the trace of $Q: H=1 / 2\left(1 / R_{1}+1 / R_{2}\right)$. In our expressions we $J=2 H$ as mean curvature. This adoption is purely notational and will not affect the physical results. Similarly, second invariant of $Q$ is defined as Gaussian curvature: $K=1 /\left(R_{1} R_{2}\right)$.

\section{Volume fraction profiles upon approaching MP}

Let us first consider an isolated planar $\mathrm{A}_{n} / \mathrm{B}_{n}$ interface, that is an interface that does not interact with any other interface and discuss what happens when we add the symmetric surfactants to this system. There is one interaction parameter $\chi$, which can be interpreted as an inverse temperature scale. By adding a surfactant/copolymer $\mathrm{A}_{N} \mathrm{~B}_{N}$ to the system that adsorbs spontaneously we can reduce the interfacial tension. At a fixed $\chi$ we observed that increasing the surfactant bulk concentration will lead to a saturated interface with zero tension. The (for microemulsions) relevant equilibrium point is characterized by this tensionless state. By gradually decreasing $\chi$ (increasing temperature) and remaining in the saturated state (tensionless) we approach the microemulsion point (MP, where $\chi=\chi^{m}$ ). We observe that the density difference between the two phases becomes less and the width of the interface grows. Schematically this is illustrated in Fig. 1 (a,d). 
Typical SCF results for such saturated state are presented in Fig. 4.11. Panels a,b,c correspond to the case of strong segregation $\left(\chi \gg \chi^{m}\right)$, d,e,f are for weaker segregation $\left(\chi\right.$ slightly higher than $\chi^{m}$ ). The density profiles of the solvents and the copolymer across the tensionless interface are shown in panels b,e. It is noticed that significantly more surfactants are needed to reach the tensionless state in the strong segregation than in weak segregation. From the density profile we can also see that the interface widens as illustrated in view-graphs $(a, d)$, and the density difference between the two bulk phases diminishes. In Fig. 1 (c,f), we report the corresponding grand potential density profiles $\omega(z)$. As the tension is zero, the $\omega$-profiles should have positive and negative 'excursions'. Moreover, the profiles are symmetric with respect of the $z_{0}$-plane, i.e. $\omega\left(z-z_{0}\right)=\omega\left(z_{0}-z\right)$. The Gaussian modulus $(\bar{\kappa})$ is the second moment of this grand potential density profile and is non-zero for both cases. In fact $\bar{\kappa}$ has a negative value for the strong segregation and a positive value for the weak segregation case.

\section{Calculation of the interfacial width}

Earlier, we observed that the interface widens upon approaching the MP. To quantify the widening it is instrumental to define interfacial width. There are several ways to define interfacial width. To avoid ambiguity we explain the way we have defined and calculated the interfacial width in our results. It should be noted, however, the physics remains generic irrespective of the way the interfacial width is defined. In our results, interfacial width $W$ is calculated from the volume fraction profiles of the bulk phases (either oil or water) as follows,

$$
W=\frac{\Delta \varphi}{\varphi_{z=\frac{-1}{2}}-\varphi_{z=\frac{1}{2}}}
$$

It should be noted that volume fraction profiles of oil and water phase are symmetric, thus using either of them to compute the interfacial width should give us similar values. However, interfacial width computed from any other profile, ex. surfactant volume fraction, grand potential etc., when used to define the interfacial width will provide a quantitatively a different picture and yet remains qualitatively the same. 

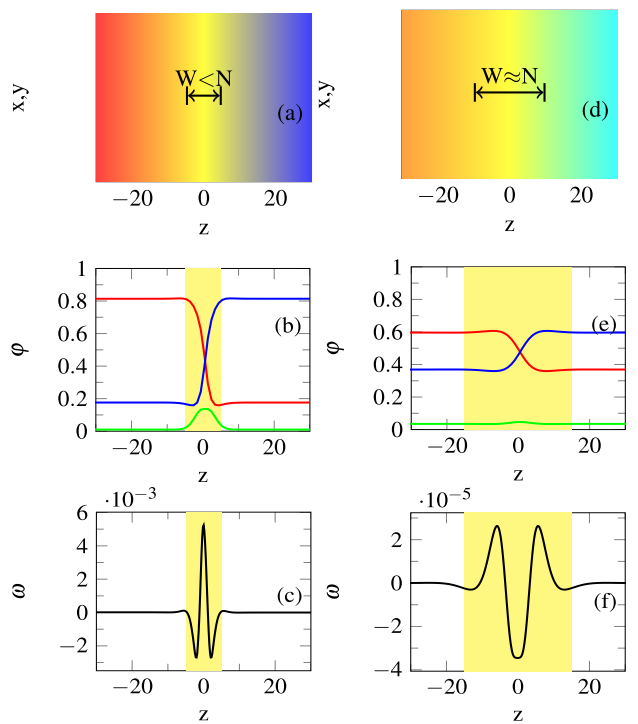

Figure 4.11: Interfacial behavior. (a,d) Graphical illustration of the density profiles across the tensionless interface with emphasis on the width $W$ of the interface and the volume fraction (density) gradients by color change color scale: red $\rightarrow$ blue: $0.8 \rightarrow 0.2,(\mathrm{~d})$ orange - cyan: $0.6 \rightarrow 0.4]$. (b,e) Selected examples for the volume fraction $\varphi$-profiles (SCF result) of bulk phases (red and blue lines) and surfactant (green line) for balanced tensionless interfaces. (c,f) corresponding grand potential density $\omega$ (minus lateral pressure) profiles across the interface. (a, b, c) far from MP $\chi=0.6,(\mathrm{~d}, \mathrm{e}, \mathrm{f})$ close to MP $\chi=0.52 . n=4$ and $N=20$. The interfacial zone is indicated by a yellow band (b, c \& e, f).

\section{Evolution of pressure profiles}

Another intersting feature that is observed upon approaching the MP is the behavior of the pressure profiles $(p(z)=-\omega(z))$. In Fig. 4.13, the evolution of pressure profile on approaching towards MP is shown. As the MP is approached the peak in the pressure profile decreases. It should also be noted that the magnitude of $y$-axis decreases drastically as the adsorbed amount of surfactant decreases. The widening of the dip in $\omega(z)$ eventually prevents the system from reaching a tensionless state. 

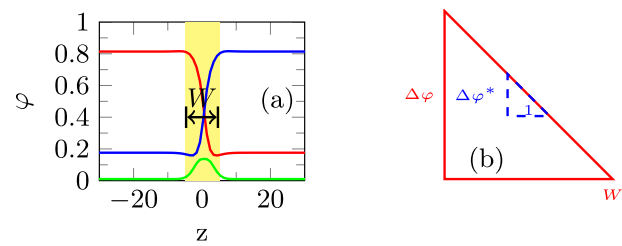

Figure 4.12: (a) Volume fraction profiles of oil (red) water(blue) and surfactants(green) far from microemulsion point $(\chi=0.6)$. (b) Similar triangles representing the width as unknown, similarity rule provides us with the equation 4.11.
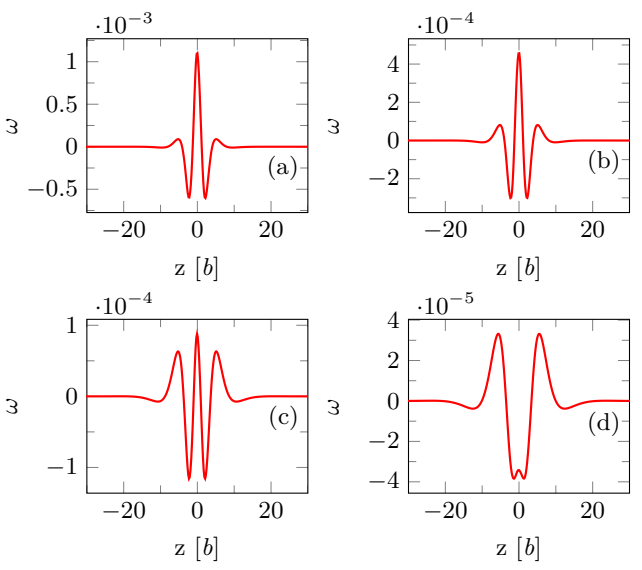

Figure 4.13: Evolution of Pressure profiles as the Flory-huggins interaction parameter is varied to approach the microemulsion point. Results for $\chi$ values of $0.55,0.54,0.53,0.52$ in subplots $(\mathrm{a}),(\mathrm{b}),(\mathrm{c}),(\mathrm{d})$ respectively.

\section{Determination of bending rigidities}

We know that the pressure profile can be directly related to the Gaussian bending rigidity of the interfaces. It is of natural interest to understand the behavior of bending rigities along the saturation line. In the main text we present the result, in this section we will briefly outlay the computational complexities associated with the estimation of the bending rigidities. For interested reader we refer our earlier article [64], in which we had published our results on scale invariant surfaces to 
unambiguously determine the bending rigidities of surfacted liquid liquid interfaces. In this section, we will provide a brief overview of the procedure and the essential details involved in direct estimation of the gaussain bending modulus from Im $3 \mathrm{~m}$ cubic phase.

\section{Computational methodology}

For a tensionless interface $(\gamma(0,0)=0)$ in saddle shape, with zero mean curvature $(J=0)$ and spontaneous curvature $\left(J_{0}=0\right)$, the energy of the interface (single Reimann manifold) from Helfrich's equation, can be shown as

$$
\iint \gamma_{G}(K) d A=\bar{k} \iint K d A
$$

Now we use Gauss-Bonnet theorem for compact, boundary-less Riemann manifold. Theorem states integral of curvature over area of the manifold is equal to $2 \pi$ times the Euler characteristic of the manifold $[95,96]$

$$
\iint K d A=4 \pi(1-g)
$$

where $\mathrm{g}$ is the genus of the manifold. For Im3m cubic phase the genus $(\mathrm{g})$ is 3 . Thus for an interface in saddle shape, interfacial energy is $-8 \pi$ times the Gaussian bending modulus $(\bar{k})$

$$
\Omega_{I m 3 m}^{s}=-8 \pi \bar{k}
$$

It is clear from equation 4.14 that interface with positive Gaussian bending modulus $(\bar{k}>0)$ prefers to form handles and complicated shapes, consistent with findings reported in literature [45].

To numerically generate such saddle shape in mean field approach, a special strategy is required.We imposed special boundary conditions that directs the system towards the saddle configuration, which is used as an initial guess for the calculations. As soon as initial guess is generated, Symmetry or mirror boundary condition is forced on 6 faces of 3D-Box or elementary box. Since by generating $1 / 8$ of the unit cell, we ensure using symmetry boundary conditions, the effects of entire unit cell is captured. All calculations are performed in double precision so that the thermodynamical quantities obtained are not affected by round-off errors. It should also be noted since we only calculate $(1 / 8)^{t h}$ of the manifold, the energy of the interface should be normalized by a factor of 8 .

$$
\left[\Omega_{I m 3 m}^{s}\right]_{3 D-B o x}=-\pi \bar{k}
$$


It could be observed that the saddle shaped interface, inferred as two dimensional Riemann manifold without boundary, crosses all the faces. These crossings are signature of handle formation in the system, unlike planar interface where the interface or manifold crosses only 4 faces. Since we are trying to represent a complex saddle shaped manifold in a Cartesian lattice, lattice artifacts become unavoidable in our calculations. Due care has been taken to reduce the lattice artifacts, by optimizing the system size. A quantitative measure of tendency of any surface to deviate from spherical shape is provided by Willmore energy $(W)$

$$
W=\frac{1}{4} \iint J^{2} d A-\iint K d A
$$

Willmore energy [97], of an ideal Im3m cubic phase, cf. equation 4.16, contains only Gaussian curvature energy, as mean curvature is zero $(J=0)$. In calculations, representation of $\operatorname{Im} 3 \mathrm{~m}$ phase in a discretized Euclidean space gives rise to contribution in Willmore energy $(W)$ from residual mean curvature $(J \approx 0)$. With increase in box size, the residual mean curvature energy increases whereas the surface in discretized space is better represented and vice versa. Hence the box size in calculations are optimized to ensure that surface is better represented with residual mean curvature energy remains negligible. From previous experiments and experience [55], the optimal value of box size for a surfactant chain-length $(N=30)$ is found to be $m_{x}=m_{y}=m_{z}=m=50$.

Similar to Im3m cubic phase, torus with a major radius $\sqrt{2}$ times its minor radius (minimal torus) has contribution only from mean curvature as the Gaussian curvature contribution nullifies. By estimating the grand potential of minimal torus mean bending rigidity of the system can be directly estimated as $\Omega_{t}=$ $\frac{1}{2} \kappa \int_{M} J^{2} d A=2 \kappa W=4 \pi^{2} \kappa$.

\section{The inverse DLVO interaction potential}

It was found that the interaction potential between two surfactant-loaded tensionless interfaces had a pronounced non-monotonic form, which was termed inverse DLVO because it was suggested that it could be the combination of two contributions: one from the interaction between two (bare) L/L interfaces and another one coming from the steric repulsion of overlapping surfactant moieties. The first contribution comes from the overlap of two tanh-like density profiles and hense must give an exponentially dependent attraction. The second contribution is expected to give a (repulsive) power-law like interaction curve. The situation is similar as in DLVO but opposite. In DLVO the attraction is due to van der Waals interactions which is known to be power-law like, while the repulsion is due to the overlap of Gouy- 
Chapman layers. We will treat both contributions of the inverse DLVO potential in order.

\section{Interaction of L-L interfaces}

In Fig. 4.14(a), the free energy of interaction per unit area, $F^{i n t}(d)=\gamma(d)-\gamma(\infty)$, between pure liquid liquid interface (represented by $\mathrm{A}_{4} / \mathrm{B}_{4}$ ) is presented for three values of the interaction parameter $\chi$. As mentioned in the main text, the length scale of the exponential attraction depends on the width of the interface $(W)$ and therefore the same results are presented as in $\log \left(F^{\text {int }}\right.$ as a function of $d / W$ in Fig. 4.14(b). It is obvious from Fig. 4.14(a) that the pure interfaces are inherently attractive. In panel b) the curves for $\chi=0.51,0.505,0.501$ (red to green) do not exactly fall on top of each other because the SCF results follow the analytical Van der Waals tanh prediction only very close to the critical point. The leveling off of $\log \left(F^{\text {int }}\right.$ at very small $d$ is due to the fact that overlapping profiles are only exponential in their tails. The leveling of at $d>>W$ it due to the limited numerically accuracy of the computations.

\section{Interaction between two surfaces covered by end-tethered chains}

Amphiphilic copolymers adsorbing on a liquid/liquid interface form a two-sided brush. To understand the steric contribution that is found when two of such layers overlap, we consider a pair of solid surfaces a distance $d$ apart. Onto each of these we have tethered linear chains with their ends. The chain length $N$, the grafting density $\sigma$ (number of chains per unit area) and the solvent quality are the main parameters. We choose the surfaces to be ideal, that is that the segments have athermal interactions. Also the solvent quality is set to the athermal value leaving just two variables $(N$ and $\sigma$ ). The generic trends found for this problem are best seen for large values of $N$. We can distinguish two limits. The brush limit $\sigma N>1$ and the mushroom limit $\sigma N<1$.

The free energy of interaction between two brushes is computed from the free energy partial open, $F^{p o}$, which is given $F^{p o}=F-\mu_{s} n_{s}=\gamma+\mu_{p} \sigma_{p}$ (where the sub index s, p refers to the solvent or polymer, $\mu$ is the chemical potential and $n$ is the number of solvent molecules in the system per unit area and $\sigma$ the grafting density. The free energy of interaction per unit area is $F^{i n t}(d)=F^{p o}(d)-F^{p o}(\infty)$.

We present the free energy of interaction for a selection of end-grafted polymer layers $A_{N}, N=10,100,1000$ at the grafting density $\sigma=0.1,0.01,0.001(\theta=1)$ 

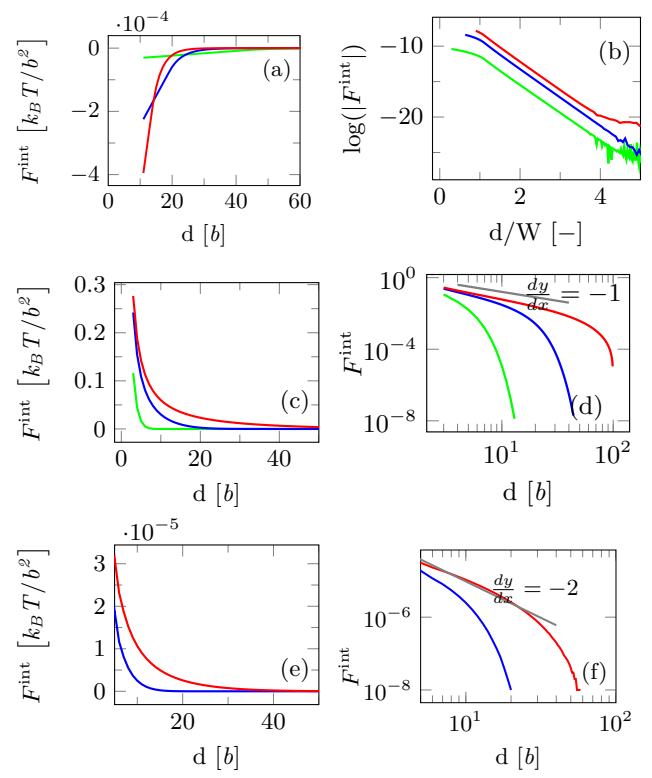

Figure 4.14: Interaction energies as function of distance between the interfaces. (a) \& (b) Pure Liquid Liquid $\left(A_{4} / B_{4}\right)$ interface with $\chi_{A B}=0.51$ (red), 0.505 (blue), 0.501 (green). (c) \& (d) Interaction free energy of polymer brushes $A_{1000}$ (red), $A_{100}$ (blue), $A_{10}$ (green) at $\theta=\sigma N=1.0$ (close to the brush limit). (e) \& (f) Interaction free energy of polymer grafted to surfaces in mushroom regime $A_{1000}$ (red), $A_{100}$ (blue) at $\theta=0.001$ (mushroom limit).

and polymers $N=100,1000$ at much lower grafting density $\sigma=10^{-5}, 10^{-6}$ $\left(\theta=10^{-3}\right)$ in Fig. 4.14(c)-(f) both in lin-lin Fig. 4.14(c),(e) as well as in log-log coordinates Fig. 4.14(d),(f). From these graphs it is clear that polymers grafted to the surface have a power-law like steric repulsion for small values of $d$ (for large $d$ there is an exponential repulsion). In the brush regime (cf Fig. 4.14(d)) the exponent is approaching -1 . We understand this dependence from the increase of repulsive segment-segment interactions: $F(d) \propto \beta \sigma N \phi(d)$, where $\beta \sim 1$ is a segment virial coefficient and $\phi$ is the (average) segment density in the brush. The latter increases when $d$ decreases: $\phi=2 N \sigma / d$ where the 2 comes from the fact that we have two brushes in contact. We also understand why the -1 coefficient is not exactly reached. This is due to the recovery of the conformational entropy loss when the stretched chains retract to their Gaussian size. This softens the 
repulsion somewhat. For short chains the effective power-law slope deviates more from the -1 because the brush is less developed. In the brush regime the onset of the interactions is proportional to the brush height $H=N \sigma^{1 / 3}$, and therefore the $N=1000$ curve extends further than the $N=100$ which in turn extends further than $N=10$ brush.

In Fig. 4.14(e),(f), the interaction energy for the polymer mushroom regime is presented as function of distance between the surfaces in linear and log-log scale respectively. In the mushroom regime as observed in Fig. 4.14(f) the exponent is -2 . The onset of the interaction is at $d \propto \sqrt{N}(d \approx 32$ for $N=1000, d \approx 10$ for $N=100$ ). Compression of mushrooms is thus less long-ranged and significantly weaker than for brushes. We can understand this exponent as follows. When $d<R_{g}$ the confined mushroom splits up in $N / g$ confinement blobs, each consisting of $g$ monomers. In the blob the chain remains Gaussian, i.e. $g \propto d^{2}$. The number of blobs per chain gives a free energy of interaction per chain: $F=N / d^{2}$ and thus the free energy interaction per unit area is $F^{i n t}=\sigma N / d^{2}$. Again we see that for the long chain the power-law dependence is better observed than for the short ones. Nevertheless, in the relevant part of the interaction curve the steric repulsion is power-law like as anticipated.

Thus in the strong segregation regime the attraction from the pure liquid-liquid interface is short-ranged and the polymers adsorbed in the interface behaves like a polymer brush leading to repulsive interaction with a local minimum observed at very small $d$. However, closer to the critical point the competing attraction from the pure liquid liquid interface and the polymer mushrooms creates a non-monotonous inverse DLVO like interaction with a local minimum observed at a higher $d \approx 2 W$. Combining systems Fig. 4.14(a),(e) is more likely to produce a non-monotonous inverse DLVO potential compared to system that combine Fig. 4.14(a),(c).

In reality we must anticipate that other contributions will add to the inverse DLVO potential. For example we will have additional undulation repulsion due to the fluctuations of the shape of the interface. Also long range van der Waals attraction must be added. Both of these contributions are power-law like and have the same $d$-dependence. Hence, these will not introduce a new length scale in the problem. Another important issue is the topological stability. In the interaction curves discussed in this section, we have assume a lamellar topology. In many cases, especially when $\bar{\kappa}>0$, we should expect a sponge-like topology. To understand how the inverse DLVO potential influences the length scale in a sponge-like topology remains an interesting issue, which might be solved using appropriate computer simulations. These simulations will go beyond the mean field level and are high on our research agenda.

Within the mean field scenario, the local minimum in the inverse DLVO potential was found the remain positive, that is the local minimum was found 
to be metastable with respect to the interfaces being far apart. This meta-stability can be influenced easily by incorporating the (mentioned) missing interaction contributions (undulations, van der Waals, etc.). We therefore believe that the inverse DLVO potential is a key result that may help us to rationalise the Winsor III scenario, which is well known to occur experimentally. 


\section{CHAPTER 5}

\section{Imposing height undulations on the liquid-liquid interface}

Interfaces that separate two de-mixed phases, one rich in $\mathrm{A}$ and the other rich in $\mathrm{B}$, have thermally excited undulations. It is generally expected that height fluctuations of interfaces are damped by the surface tension $\gamma$, which resists against area increase, and the mean bending modulus $\kappa$, which goes against strong curvatures. For a simple regular solution-like lattice model we know both $\gamma$ and $\kappa$ from a free energy analysis of homogeneously weakly curved interfaces. Here we explore a hybrid Monte Carlo self-consistent field (MC-SCF) approach to study undulating interfaces in 3D to find out whether the truly fluctuating interface is indeed controlled by the known $\gamma$ and $\kappa$. We define the location of the interface as the points where the density of component A equals that of component B. In the ground state the interface is flat $(h(x, y)=0$ for all $(x, y))$. With an MC protocol, we generate for a specified set of $\{x-y\}$ coordinates a height $h(x, y)$ away from the ground state value. Operationally, these heights translate in the SCF machinery into a set of Lagrange parameters which enforce the local equality of the densities of the A and B component. Then the SCF-machinery finds the best interface draping through this specified set of constrained locations. The grand potential of such constrained SCF-solution serves as internal energy in the MC algorithm accepting or rejecting a snapshot based on the metropolis algorithm. Our results indicate that alongside $\gamma, \kappa$, the Gaussian bending modulus also plays a role in interface fluctuations. We attribute this to the fact that periodic boundaries are imposed on the piece of the interface that is studied. The integral of the Gaussian curvature over this representative piece of the interface does not vanish. We prove the feasibility of the hybrid MC-SCF approach to study interface fluctuations within the limits of discretization issues prevailing at the current state of the art. 


\subsection{Introduction}

A mixture of two incompatible components, even when thoroughly mixed, separates in time into two distinct phases. The interface at the boundaries between the two de-mixed phases has fascinatingly complex properties and inspired research dating back to Van der Waals[150]. Every day, people encounter such systems, e.g., while cooking and eating food products $[9,151,152]$. Large oil companies have to deal with such systems as these occur in oil reservoirs and during disaster management when oil-spills occur[153]. Interfaces are important to understand life and living organisms as interfaces are omnipresent. One may find interfaces not only in our intestines but also inside our cells. Understanding interfaces, therefore, remains a relevant topic of research in countless disciplines. The science of interfaces is blessed with many interesting questions.

One cannot say that interfaces are poorly understood, yet many questions wait for an answer, even for the most simple interfaces that we can think of. To develop quantitatively accurate models for interfaces requires capturing the density gradients when going from one phase to the other and evaluate all excess quantities that are associated with the presence of such interface. On top of this, we need to understand how thermal fluctuations manifest at these interfaces. Undulatory fluctuations exist up to the nanoscale, that is, up to the length scale of the molecules involved. (for eg. on a sea, one could see these as waves[154])

We, through a series of papers, have proposed an efficient equilibrium-route to model such interfaces using Scheutjens-Fleer self-consistent field theory.[64, 134, 155] In this mean-field approach we can obtain insight about the density gradients, the interfacial tension, the density difference between the bulk phases, etcetera upon the approach of the binary system towards its critical point. Because of the mean-field approximation, interfaces in this approach remain boringly flat (these show no undulation fluctuations) and this allows the reduction of the analysis to one-gradient models. In such models, we can, for a given value of the chemical potentials (binodal values), curve the interface away from the planar ground state, into the spherically or cylindrically curved interfaces. The curvature dependence of the interfacial tension $\gamma$ gives information on the mechanical parameters of the interface in an equation, which was first presented by Helfrich[47] to discuss the shape changes of lipid bilayer membranes[156, 157]:

$$
\gamma(J, K)=\gamma(0,0)-J_{0} \kappa J+\frac{1}{2} \kappa J^{2}+\bar{\kappa} K+\mathcal{O}\left(1 / R^{3}\right)
$$

Here the mean curvature $J=1 / R_{1}+1 / R_{2}$, the Gaussian curvature $K=$ $1 / R_{1} \times 1 / R_{2}$, with $1 / R_{1}$ and $1 / R_{2}$ being the principal curvatures that characterize the shape of the interface at a specified point along the surface (for homogeneously curved interface these curvatures are trivially identified). $J_{0}$ is the spontaneous curvature. The mean bending modulus, defined as $\kappa \equiv\left(\partial \gamma / \partial J^{2}\right)_{0,0}$, is believed to 
control (in part) the out of plane fluctuations of the interface and $\bar{\kappa} \equiv(\partial \gamma / \partial K)_{0,0}$ is a parameter that controls the topology of interfaces. A negative value for $\bar{\kappa}$ promotes similar signs of the two principal curvatures, whereas a positive value promotes opposite signs for these quantities (as these occur in saddles)[158]. Accurate evaluation of the mechanical parameters requires that the derivatives of $\gamma$ are evaluated for weakly curved interfaces and at fixed chemical potentials of the molecular components (identical to the value found to exist for the planar ground state).

Focusing on purely symmetric systems (the two molecular components have the same molar volume), for which in the SCF model the interfaces have a zero spontaneous curvature $J_{0}=0$ by construction, we have shown in a recent paper that there exist a length scale $\xi=\sqrt{\frac{\kappa}{\gamma}}$, which appears to take a value of the order of the molecule size, (virtually) independent on the value for the interaction parameter[134]. Hence even when the system goes towards its critical value where the interfacial width diverges, the density difference between the two phases vanishes and where the interfacial tension vanishes, the length scale $\xi$ is almost a constant. The physical interpretation that presents itself is that the out-of-plane fluctuations with a wavelength $\lambda$ are controlled by the value of the interfacial tension $\gamma$ when $\lambda>\xi$, while when the $\lambda<\xi$ the out-of-plane fluctuations are damped by the mean bending modulus[159, 160].

There is an interesting dilemma associated with this interpretation. In SF-SCF, we have evaluated the mean bending modulus in the weak curvature limit. The mean bending modulus, however, has a role to modulate the out-of-plane fluctuations for the high curvature limit (at small wavelengths)[161]. It is not evident that indeed the weak curvature limit result may be applied to strongly curved interfaces. Thus, the true role of the mean bending modulus, even for such simple fluctuating interfaces, remains uncertain. To clarify this, we need to consider 3D systems where the interface (an 'object' in 2D) fluctuates because of undulations that arise from a molecular-picture and the mechanical parameters are emerging (not imposed).

This sets up the requirement for the current work. Here we like to account for the thermal fluctuations and study the undulatory height variations of a model liquidliquid interface, as it occurs in the Scheutjens-Fleer self-consistent field theoretical (SF-SCF) approach. We note, to date there are no such attempts presented in the literature to generate undulatory interfaces using a regular-solution-like model.

To this end, we introduce a hybrid Monte Carlo SCF (MC-SCF) method. This approach combines sampling of a number of degrees of freedom with the $\mathrm{MC}$ protocol. It passes these degrees of freedom on to the SF-SCF machinery, which solves (constrained) SF-SCF equations for the liquid-liquid interface. More specifically the MC degrees of freedom are so-called 'pinning'-constraints. With the use of a Lagrange parameter per pinning constraint, we can force the interface to pass trough this pinning point (the density of both molecules are forced to be equal 
at such point.) The grand potential of the system with the specified pinning points takes the role of the internal energy of the system in the Metropolis algorithm. Simple translational moves plus wave-like cooperative modes for the pinning points are implemented to sample the Boltzmann distribution for the fluctuating interface. Similar hybrid methods have been introduced by our group earlier for gels of neutral telechelics[162-164] and gels of poly-electrolyte chains near a surface [165].

With the MC-SCF method we expect to find relevant 'snapshots' of the shape of fluctuating/undulating liquid-liquid interface. From this we expect to be able to estimate the governing interfacial tension and the mean bending modulus. How to establish ways to do this is part of the current work. Finally, in this work, we show that two factors prevent us from achieving direct comparison of weakcurvature rigidity values to that of the undulatory interface: (i) Presence of influence from the Gaussian bending modulus (ii) Discretization issues leading to a loss of high-curvature resolution.

First, let's discuss how an influence from $\bar{\kappa}$ arises in simulations. It is well known that the Euler characteristic of a piece of a flat interface (with periodic bounds) is $\chi=1[166]$. Further, $\int K d A$ is zero as $K$ is zero at each point. Similarly $\int J d A=0$ as well as $\int J^{2} d A$. According to Gauss-Bonnet theorem, for a flat interface $\int K d A+\int k_{g} d s=2 \pi \chi[55,167]$. The second integral over the geodesic curvature reduces to the sum over the four corners (giving $2 \pi$ ).

Upon addition of interface undulations, it is well known that the Euler characteristic does not change[168]. However, the integral over the geodesic curvature $\left(\int k_{g} d s\right)$ for an arbitrarily curved interfaces is not constant. To compensate for this variation the interface develops spatial variations of the Gaussian curvature and the $\int K d A$ varies in concert with the geodesic curvature integral such that the Euler characteristic remains unity. Our MC-SCF results are in line with this and we will show that the integral over the Gaussian curvature differs from snapshot to snapshot in our results.

It is instructive to elaborate a bit more on this issue. The grand potential that is associated to the interface (in a particular configuration) follows (numerically) from the SCF machinery and is evaluated without the need to know the position of the interface: it does not need any evaluation of the curvatures (see below). However, as soon as the interface position is traced we may evaluate both the area of the interface as well as the local curvatures (see below) and decompose the grand potential into contribution from its components. Hence we can (i) evaluate the contribution from the area term $\int \gamma d A$, (ii) the contribution that contains the mean curvature $-J_{0} \kappa \int J d A$, (iii) a term containing the mean curvature squared $\frac{1}{2} \kappa \int J^{2} d A$ and (iv) one for the Gaussian curvature $\bar{\kappa} \int K d A$. Focusing on symmetric system that by construction have a vanishing spontaneous curvature, $J_{0}=0$, the integral over the mean curvature has no effect. When the topology of the interface did not change, it is generally assumed that the integral over the Gaussian curvature remains zero. 
However, above we argued that this is not the case in our simulation system and we expect a finite contribution of it. We also expect a contribution from the integral over the mean curvature squared. Hence, there are three parameters that play a role, $\gamma, \kappa$ and $\bar{\kappa}$ in the grand potential of the systems (which serves as an internal energy in the Metropolis algorithm)

The analysis of interfacial fluctuations assumes typically only contributions from $\gamma$ and $\kappa[33,42,85,169]$. Our working hypothesis therefore is that the classical ideas to analyse undulating interfaces, taking only $\gamma$ and $\kappa$ into account, will work best for systems that have $\bar{\kappa} \approx 0$ and becomes systematically worse when the Gaussian bending rigidity deviates more from zero. We cautiously note that the contribution of Gaussian curvature to interfacial fluctuations is not only bound to simulations but also in experimental systems with non-zero geodesic curvature.

The second factor, as said above, is the discreteness of the simulation lattice sites. In the MC-SCF we can place the pinning points only at discrete values (at lattice sites). As a consequence, it is necessary to place the pinning points some distance away from each other so that the SCF equations could realistically find an interface connecting through the pinning points. The SCF theory curves the interface smoothly through the pinning points, and as a result, we lose undulatory modes with a wavelength that is shorter than the distance between the pinning points. To date, this limits the resolution of the current simulations somewhat and in the discussion, we will return to this issue. However, recent progress[170] from our group shows that a more refined lattice can be adapted for the presented hybrid MC-SCF in future.

To summarize, within the limits of the two influencing factors, we show: (i) provided the right rigidity parameters a membrane model can realistically reproduce the undulatory interfaces (ii) all three parameters $\gamma, \kappa$ and $\bar{\kappa}$ influence the fluctuations of symmetric interface located within a periodically bounded simulation box (iii) a realistic comparison of rigidity parameter at weak-curvature and strongcurvature is only possible when the simulations are performed in a parametric space where $\bar{\kappa}=0$ and discreteness of lattice is addressed. The remainder of this paper is as follows. In the method section, we will go into sufficient detail about how the MC-SCF has been implemented. In the results section, we will give insight into our main results. In the Discussion, we will go into more detail about what pros and cons we have encountered. In the summary, we collect our major findings.

\subsection{Method}

Focusing on the main steps we start with an introduction to the Scheutjens-Fleer self-consistent field theory (SF-SCF). While doing this we decorate this with the necessary details of how in the hybrid Monte Carlo Self-consistent field (MC-SCF) method the pinning constraints are implemented. In a separate subsection, we give 
details on the Monte Carlo protocol that is combined with the SF-SCF method. This is followed by a section wherein we detail how we have analyzed the performance of the MC-SCF hybrid. The results and discussion section is split into two parts. In the first one, we work out a case study for a simple system. In the second part, we give insight into the main results as a function of a few relevant variations of our parameters. The paper is concluded as usual by a summary of our major findings.

\section{Scheutjens-Fleer Self Consistent Field Theory extended with pinning constraints}

To implement a self-consistent field model we combine the freely jointed chain model with a Flory-Huggins equation of state. This is done on a lattice. Scheutjens and Fleer suggested using the segment size $b$ equal to the lattice site length $b$ so that there is just one discretization length in the system. [53, 54]. In the system we have $x=1,2, \cdots, M_{x}$ coordinates in the $x$-direction, $y=1,2, \cdots, M_{y}$ in the $y$-direction and $z=1,2, \cdots, M_{z}$ in the $z$-direction. The volume is $V=M_{x} \times M_{y} \times M_{z}$ lattice sites. Below, a coordinate is given by the notation $r=(x, y, z)$. In the z-direction, we have mirror-like boundary conditions and we have periodic boundary conditions in the two other directions.

Two liquids are modeled as linear strings of $N$ segments of type $A$ and $B$, respectively. We note though the number of segments, $1 \leq N \leq \infty$, the choice of $N$ is restricted by computational complexity (Larger $\mathrm{N}$ leads to larger computational time) Segments along the chain are referred to by their ranking number $s=1,2, \cdots, N$. On top of this, we will insist on equal amounts for the two components (Note, this condition is not required when we enforce pinning constraints). An effective repulsion between the segments is quantified by specifying a Flory-Huggins interaction parameter $\chi=\frac{Z}{2 k_{B} T}\left[2 U_{A B}-U_{A A}-U_{B B}\right]$ where $U_{X Y}$ is the interaction energy for a contact between $X$ and $Y$. To estimate the number of contacts between $\mathrm{A}$ and B the Bragg Williams mean-field ansatz is used, namely that the chances that A and $\mathrm{B}$ meet in a homogeneous phase are equal to the product of the respective volume fractions. This is an approximation because when A and B repel each other the distributions should be adjusted such that the chance that they meet is reduced compared to the random distribution and the inverse happens when they are attracted to each other. These correlations are ignored, similarly as in regular solution and the Flory-Huggins theory.

In SF-SCF we write a mean field free energy (i.e. the Helmholtz energy in units of $k_{B} T$ ) for a molecularly inhomogeneous system [55-58] in terms of volume fraction $\varphi_{x}(r)$ and complementary segment potential $u_{x}(r)$ profiles for segment types $x=A, B$. Equilibrium SCF solutions involves optimizing the free energy $(F)$ with respect to its variables, respectively the segment potentials and the volume fractions. 
To facilitate proper extremization we add Lagrange parameters. The set of parameters $\alpha(r)$, is added to implement the incompressibility constraint, $\varphi_{A}(r)+\varphi_{B}(r)=1$, applicable in each coordinate $r$. In the MC-SCF hybrid we have in addition introduced parameters $\Delta_{r ;\left[r_{0}^{M C}\right]} \equiv \delta_{r ;\left[r_{0}^{M C}\right]} \nu(r)$, where $\delta_{r ;\left[r_{0}^{M C}\right]}$ is unity when $r \in\left[r_{0}^{M C}\right]$ and zero otherwise and $\nu(r)$ is a Lagrange field that enforces that the densities (volume fractions) of both components $\mathrm{A}$ and $\mathrm{B}$ are equal to each other (this only happens at coordinates $\left[r_{0}^{M C}\right]$ of course)

$$
\begin{array}{r}
F=-\ln Q([u])-\sum_{X, r} u_{X}(r) \varphi_{X}(r)+F^{i n t}([\varphi])+ \\
\sum_{r} \alpha(r)\left[\sum_{X} \varphi_{X}(r)-1\right]+\Delta_{r ;\left[r_{0}^{M C}\right]}\left[\varphi_{A}(r)-\varphi_{B}(r)\right]
\end{array}
$$

We will comment on this free energy functional term by term. The first term features the system partition function $Q$. In the mean-field approach one can decompose it into molecular partition function $Q=\Pi_{i} q_{i}^{n_{i}} / n_{i}$ ! where $i=1,2$ refers to $A_{N}$ and $B_{N}$ respectively. The molecular partition function $q_{i}$ contains the statistical weight of all possible and allowed conformations of molecule $i$ (see below). $n_{i}$ is the number of molecules of type $i$ in the system.

The second term in 5.2 transforms the free energy from potential space to density space. This term complicates the 'optimisation' of 5.2. It turns out that the free energy is minimized w.r.t the densities (volume fractions) but maximized w.r.t the potentials. The third term gives the interaction terms (in segment density 'space'). This term is needed because the sum of the two first terms results in the entropic terms of the free energy only. For the interactions, we take the classical Flory-Huggins equation of state generalized to account for the gradients in density.

$$
F^{i n t}=\sum_{r} \chi \varphi_{A}(r)\left[\left\langle\varphi_{B}(r)\right\rangle-\varphi_{B}^{b}\right]
$$

As we have only two segment types in our system, there is only one interaction parameter $\chi$. The $\varphi_{B}^{b}$ refers to the bulk volume fraction of B (of one of the bulk phases) and ensures normalization of the segment potentials (by ensuring that the $F^{i n t}$ is zero in the reference phase). The angular brackets are needed to properly account for the interactions and take effect only when there are density gradients in the system.

As mentioned the angular brackets are needed to account for the contact energy in a system with gradients in density. In our earlier work, we proved the importance of such a term. It was found that neglecting such contribution leads to erroneous contributions to the curvature derivatives of the free energy (and thus the error features in the bending rigidities of the interface)[134]. This term has a direct influence on how interfaces fluctuate. Mean-field theories often neglected such 
contribution, overlooked its importance, and reported physically unacceptable negative values of the mean bending rigidity. The Cahn-Hilliard theory [45] already stresses the importance of the 'curvature' contribution in the segment potentials. For the three gradients coordinate system system with $r=(x, y, z)$ we may write[53, 55] for a simple cubic lattice

$$
\begin{array}{r}
\left\langle\varphi_{B}(r)\right\rangle=\left(\varphi_{B}(x-1, y, z)+\varphi_{B}(x+1, y, z)+\right. \\
\varphi_{B}(x, y-1, z)+\varphi_{B}(x, y+1, z)+ \\
\left.\varphi_{B}(x, y, z-1)+\varphi_{B}(x, y, z+1)\right) / 6 \\
=\varphi_{B}(r)+\frac{1}{6} \nabla^{2} \varphi_{B}(r)
\end{array}
$$

In the last part of the 5.4, we give the continuum interpretation of the angular brackets.

The fourth term in 5.2 is introduced so that the extremization of the Helmholtz energy obeys the incompressibility constraint. At each coordinate, we force that the sum of the densities of $\mathrm{A}$ and $\mathrm{B}$ equal unity (no vacancies).

The final term in 5.2 is needed in the MC-SCF hybrid to force the interface away from the mean-field exactly planar state. As we will discuss below the set of coordinates onto which this term enforces that the density of A equals that of $\mathrm{B}$, is generated by a Monte Carlo protocol.

The SCF protocol requires subsequently that a saddle point is found for the Helmholtz energy (Eqn. 2). When $\partial F / \partial \varphi_{A}(r)=0$, we find that the potentials must obey

$$
\begin{aligned}
& u_{A}(r)=\alpha(r)+\Delta_{r ;\left[r_{0}^{M C}\right]}+\chi\left(\left\langle\varphi_{B}(r)\right\rangle-\varphi_{B}^{b}\right) \\
& u_{B}(r)=\alpha(r)-\Delta_{r ;\left[r_{0}^{M C}\right]}+\chi\left(\left\langle\varphi_{A}(r)\right\rangle-\varphi_{A}^{b}\right)
\end{aligned}
$$

Note that these potentials are by construction dimensionless, that is they are given in units of $k_{B} T$. It is important to note the sign change for the $\Delta$ Lagrange field for A and B segments, respectively.

Setting $\partial F / \partial u_{X}(r)=0$ shows the way to evaluate the densities: $\varphi_{X}(r)=$ $-\partial \ln Q / \partial u_{X}(r)$. As the molecular partition function for molecule type A is going to depend on the segment potentials of the segment type A, and vice versa for the B-molecule, we can implement this optimisation per molecule. The molecular partition functions are found from the endpoint distribution $q_{i}=\sum_{r} G_{i}(r, N \mid 1)$. The end-point distributions are recursively found from $G_{i}(r)=\exp \left(-u_{i}(r)\right)$ by the propagator $G_{i}(r, s \mid 1)=G_{i}(r)\left\langle G_{i}(r, s-1 \mid 1)\right\rangle$, where the angular brackets have the same meaning as in 5.4. The free segment distribution simply is the Boltzmann equation for the specified segment: $G_{1}(r) \equiv G_{A}(r)=\exp \left(-u_{A}(r)\right)$ and the same applies for the molecule $i=2$ that has segment type B. The 
segment densities are found by the composition law, which for homopolymers read: $\varphi_{i}(r)=\sum_{s=1}^{N} C_{i} G_{i}(r, s \mid 1) G_{i}(r, N-s+1 \mid 1) / G_{i}(r)$.

The normalization constant $C_{i}$ depends on the mode of operation. In the MCSCF the position of the interface is controlled by a Lagrange parameter $\Delta_{r ; r_{0}}^{M C}$, we no longer need to specify a fixed amount of one of the components (as is needed in a canonical ensemble), but we can normalize the densities with $C_{i}=\varphi_{i}^{b} / N_{i}$ where $\varphi_{i}^{b}$ is specified by the binodal: A proper binodal value is a (relevant) root of the Flory-Huggins Eqn. $\ln \frac{\varphi}{1-\varphi}+\chi N(1-2 \varphi)=0$.

In all other cases, i.e. without the pinning constraints, we have set the amount of $\mathrm{A}$ equal to the amount of $\mathrm{B}$, so that the interface splits the volume in two equally sized phases. Then, the volume fractions for component A are normalised using $C_{A}=\frac{1}{2} \frac{V}{N q_{A}}$. The molecular partition function $q_{A}=\sum_{r} G_{1}(r, N \mid 1)$. For the solvent (molecule B), we use the normalisation $C_{B}=1-N C_{A}$, which follows from the requirement that $\varphi_{A}^{b}+\varphi_{B}^{b}=1$ and $\varphi_{A}^{b}=N C_{1}$.

The optimisation of the Helmholtz energy with respect to the Lagrange field $\alpha$ tells us that the SCF solution should obey to the incompressibility constraint. This optimisation is done numerically using an iteration. If during this iteration we find that the system does not obey to the incompressibility constraint, update $\alpha(r) \leftarrow \alpha(r)+\varphi_{A}(r)+\varphi_{B}(r)-1$. The same type of update is performed when the SCF solution does not yet obey to the pinning constraint. The $\nu\left(r_{0}^{M C}\right) \leftarrow \nu\left(r_{0}^{M C}\right)+\varphi_{A}\left(r_{0}^{M C}\right)-\varphi_{B}\left(r_{0}^{M C}\right)$.

Numerical solutions that optimize the free energy functional and obey all constraints have the property that the potentials both determine and follow from the volume fractions profiles and vice versa and are said to be self-consistent. We find such SCF solution routinely up to high precision (at least 9 significant digits for $1 \mathrm{D}$ calculations and 4 significant digits for 3D calculations)

\section{Thermodynamics of equilibrium solution}

For each SCF solution one can compute the grand potential by $\Omega=\sum_{r} \omega(r)$ wherein the grand potential density at coordinate $r$ is given by $\omega(r)=-\sum_{i}\left(\varphi_{i}(r)-\varphi_{i}^{b}\right) / N_{i}-$ $\alpha(r)-\chi\left(\varphi_{A}(r)\left[\varphi_{B}(r)+\frac{1}{6} \nabla^{2} \varphi_{B}(r)\right]-\varphi_{A}^{b} \varphi_{B}^{b}\right)$. The planar (one-gradient) interface has a tension $\gamma_{p}=\sum_{z} \omega(z)$, where $z$ is the coordinate in the planar system. This planar interface serves as the ground state or reference state upon which MonteCarlo moves can be made by specifying new positions for the pinned coordinates. The following sections will describe the implementation of such procedure in detail.

\section{Implementation of Monte Carlo strategy in MC-SCF}

The Monte Carlo strategy requires (i) an initial configuration, (ii) a strategy to generate 'trials', (iii) an acceptance criterion, and (iv) equilibration. Data collection and analysis can commence after a suitable equilibration run. 


\section{Initiation}

The target of the MC train is to provide the SCF machinery with appropriate successive sets of coordinates $\left[r_{0}^{M C}\right]$. Let these coordinates be numbered $k=$ $1, \cdots, K$ where $K$ is the total number of degrees of freedom in MC and this is of course the same number as the pinning constraints in the (constrained) SCF calculations. We start with a mean-field regular solution interface in $3 \mathrm{D}$ such that the interface is located at the plane $z^{\prime}=\left(M_{z} / 2+0.5\right.$ (So when $M_{z}=49$, the interface is placed at $z^{\prime}=25$ as the initial guess for the interface is exactly half-way in the system). The $K$ degrees of freedom are chosen to $\left(x, y, z^{\prime}\right)$ sit in a square lattice grid. The distance between the points is taken to be $\Delta_{x}=\Delta_{y} \approx \xi$ the length scale suggested by $\xi=\sqrt{\kappa / \gamma}$. So the number of MC points $K=\left(M_{x} / \xi\right) \times\left(M_{y} / \xi\right)$.

The starting point for the simulation is the perfectly flat interface (as this is made available from the classical SF-SCF equations). We refer to this as the planar ground state, where the grand potential of the system $\Omega=\Omega_{0}=\left(M_{x} \times M_{y}\right) \gamma_{p}$ (the grand potential is the characteristic function during the simulations because during the simulation we fix the chemical potentials of both the $\mathrm{A}$ as well as the $\mathrm{B}$ component). When we refer to the free energy of the system $F$ it is understood that we talk in this case about the grand potential. (as the system belongs to $(\mu, V, T)$-ensemble) The initial shape of the interface can be mathematically represented by a plane $h(x, y, t=0)=0$ at the ground state, that is $h=0$ is set at $z=z^{\prime}$. How the interface is precisely located further-on in the MC train, is discussed in the parameter sections.

\section{Trial moves}

We have implemented two types of moves. Translational moves and cooperative mode-like moves. In the translational move one of the $K$ degrees of freedom is chosen randomly and displaced in $x$ - or $y$ - direction, by one lattice site. When the distance between two neighboring pinning sites is less than 3 (lattice units) the translational trial is rejected. This choice was optimal to prevent close proximity between pinning points (undesired for computational reasons - slow convergence and high probability of rejection) and to ensure a more or less homogeneous distribution of pinning points on the interface (as it was the best way to sample the interface shapes). The translational moves introduced some more variation in these shapes but we made sure that the pinning points remained more or less homogeneously distributed. In each MC move, we do $K$ of such translational trial steps. So one pinning site may be moved multiple times while others remain untouched. The translational move is combined with a cooperative move.

During the cooperative move we select for both the $x$ - as for the $y-$ direction a phase $\phi$, a wavelength $\lambda$ and an amplitude $A$. For each pinning point $k$ the $(x, y)_{k}$ coordinates are known and the $z_{k}$-coordinate is changed by 
$z_{k} \leftarrow z_{k}+A_{x} \sin \left(\lambda_{x} x+\phi_{x}\right)+A_{y} \sin \left(\lambda_{y} y+\phi_{y}\right)$ and the $z_{k}$ value is rounded to the nearest integer value. The $1<\lambda_{x}<M_{x}$, and $1<\lambda_{y}<M_{y}$, and the phase is $0<\phi<2 \pi$. When the value of the amplitude is chosen randomly $-3<A<3$ we found an acceptable acceptance probability of about $50 \%$ (See parameter tables for exact values). For each MC step, the coordinates are 'transferred' to the SCF machinery, which solves the SCF equations taking the (new) pinning constraints into account. The new SCF solution features an interface that is draped through the positions of all $K$ pinning points. In summary one MC step encompasses changes for each pinning point $k$ (on average).

\section{Metropolis algorithm}

A Metropolis-like algorithm is implemented to either accept or reject the new set of pinning points. This acceptance criterion is based on the SCF grand potential $\Omega^{\text {new }}$ in comparison with the previous value of $\Omega^{\text {old }}$. Let $\Delta \Omega=\Omega^{\text {new }}-\Omega^{\text {old }}$. When $\Delta \Omega^{\text {new }}<0$ the new set of pinning points is accepted. Otherwise it is accepted with a probability $P=\exp (-\Delta \Omega)$. When a configuration of the pinning points is rejected the actual position of the pinning points is set back to the old values.

In this Metropolis-like algorithm, the grand potential $\Omega$ serves as the 'internal energy' of a classical simulation. In $\Omega$ the relevant free energy (energy and entropy) contributions of all degrees of freedom that are not accounted for by $\mathrm{MC}$ are included.

\section{Equilibration}

As usual, the method involves an equilibration period, that is a number of MC steps to move towards the Boltzmann distribution of fluctuations that characterize equilibrium. The number of Monte Carlo moves required to reach such a state is defined as the equilibration time $t_{e q}$. The mean-field $\Omega$ fluctuates and typically moves to some higher value (due to the area increase and bending energy of the interface). After the equilibration $\Omega$ fluctuates around a stationary value $\langle\Omega\rangle$. The configurational entropy of the fluctuating interface $S_{\text {conf }}$ is not directly available from the simulations. The true surface tension in the system should account for this entropy. Hence a better estimate for the overall surface tension $\langle\gamma\rangle=\left\langle(\Omega\rangle-S_{\text {conf }} / k_{B}\right) /\left(M_{x} \times M_{y}\right)$. We expect that $\langle\gamma\rangle<\gamma_{p}$.

\subsection{Measurables}

\section{Height measurements}

For a given instance of the MC trajectory, we need to find the location of the interface: at the MC time step $t$ we express the height as $h(x, y, t)$. The interface is 
easily located by scanning the volume fraction profile in the $z$ direction for specified values of $x$ - and $y$. Far from the interface, the bulk volume fractions have binodal values. In the interfacial zone, there is a gradient from one binodal density to the other. The $z$-coordinate for which the densities of A and B are equal, $z^{\prime \prime}$, is found by interpolating the densities from layer to layer. This equal density plane may also be referred to as the Gibbs plane. The height then is found by $h(x, y, t)=\left(z^{\prime \prime}-z^{\prime}\right)$. From the height measurements, the surface area of the fluctuating interface $(\mathcal{A})$ can be estimated computationally by determining the $\alpha$-shape [171].

Further, from the $\alpha$-shape of the fluctuating interface the mean $(J)$ and Gaussian curvature $(K)$ at every point of the interface is estimated. Integrals of mean curvature, square of mean curvature and Gaussian curvature is estimated as $\mathcal{J}=\sum J d A, \mathcal{H}=\sum J^{2} d A, \mathcal{K}=\sum K d A$ over all the sites. Note, that the elemental area $d A$ is not equal to $d x \times d y$. $d A$ measures the area of an irregular quadrilateral (trapezium) connecting the interfacial heights in the neighbouring lattice sites. When the interface does not undulate (flat) the elemental area $d A=d x \times d y$.

\section{Probability density function}

Interfacial height fluctuations $h(x, y, t)$ of several independent Monte Carlo realizations are collected. Mean height of the interface can be estimated as $\bar{h}=\langle h(x, y, t)\rangle$, where the angular brackets denote sample-averaged value. It is interesting to observe how much the interfaces deviate from this mean value. By defining the deviations as $\delta h(x, y, t)=h(x, y, t)-\bar{h}$, a discrete probability density function of such deviations $p(\delta h)$ can be obtained. To obtain the discrete probability density function $\delta h(x, y, t)$ values are binned and normalized by the bin size (default value of bin size: $0.08 b)$. Higher-order moments: Variance, skewness and kurtosis are also determined (not shown).

\section{Spatial correlation function}

Another interesting measurable is the spatial two-point correlation function, $G(\delta r)$, where $\delta r$ denotes the distance between the two points, $r=x \hat{i}+y \hat{j}$ and $r+\delta r=(x+\delta x) \hat{i}+(y+\delta y) \hat{j}$ (where $\hat{i}, \hat{j}$ correspond to unit vectors in $x, y$ direction respectively). For inhomogeneous systems, it is well known that the rough internal interfaces are self-affine surfaces[172-174]. Thus, by defining the interfacial height at $r$ notionally as $h(r)$, the two-point correlation is estimated as, $G(\delta r)=\left\langle(h(r)-h(r+\delta r))^{2}\right\rangle$. Note, that

$$
G(\delta r)=2 w^{2}\left[1-\exp \left(-\left(\frac{\delta r}{\xi_{c}}\right)^{2 H}\right)\right]
$$

where $w^{2}$ is the variance (second moment of the discrete probability density function), $H$ is the Hurst exponent and $\xi_{c}$ is the correlation length $[173,175]$. 


\section{Fourier transform and Structure factor}

Finally, a discrete Fourier transformation $(h(q))$ of interfacial height $(h(r))$ is performed as follows,

$$
h(q)=\sum_{r=1}^{M} h(r) \exp (-2 \pi i r q)
$$

where $M$ denotes the box size. Recall that periodic boundaries are used in the tangential direction (to the interface). By imposing coordinated waves moves that do not fit the box size, the SCF solution, often, moves the average position of the interface slightly above or below (if the pin constraints at the end of the boxes move in the same direction for the imposed wave). Additionally, a high-frequency noise is added to the $h(r)$. A Hann-window is applied to filter such high-frequency terms. It should be noted that earlier simulation works, employ a 6 -point or 9-point box filter instead. Both methods do not provide any difference and a Hann-window is used for convenience. Our protocol to perform the fast Fourier transform is available as an open-source repository for interested readers (GitHub project: PosProInt).

A two-point correlation function can be used to determine the structure factor, $S(q) . S(q)$ in the units of $b^{4}$ is obtained by multiplying the Fourier transformed height fluctuations with the projected interfacial area, $S(q)=\left\langle h(q)^{2}\right\rangle A$. Structure factor is believed to contain the information about the rigidity parameters [176],

$$
S(q)=\frac{k_{B} T}{\gamma q^{2}+\kappa q^{4}+\cdots}
$$

\section{Estimation of bending rigidities}

Given Eq. 8, it is possible to estimate both interfacial tension and mean bending rigidity using linear regression. We can rewrite Eq. 8 as,

$$
\gamma+\kappa q^{2}=\frac{1}{S(q) q^{2}}
$$

By defining, $x=q^{2}$ and $y=1 / S(q) x$ we obtain a simple linear equation, $y=\gamma+\kappa x$ whose slope provides the mean bending rigidity and the intercept provides the interfacial tension. We will use the notations $\gamma_{M C}, \kappa_{M C}$ for such estimates and reserve the notation $\gamma_{p}, \kappa$ for their SF-SCF counterparts.

\section{Error estimation}

For the measured values, for example, when $\xi_{c}$ determined using spatial correlation analysis $n$ samples are averaged. It was found that the value of $\xi_{c}$ depended on the number of samples averaged. Assuming a normal distribution for the measurable 
as a function of $n$, the statistical uncertainty $\sigma$ of the measurable was evaluated. It should be noted, upon the limit value of $n \rightarrow \infty$ the statistical error $\sigma \rightarrow 0$. (We cautiously note, the reader to not confuse the standard deviation in statistical uncertainty $\sigma$ with a standard deviation of height fluctuations $\sqrt{w^{2}}$. The latter is a constant and will not vanish with increasing the number of samples.) However, for practical reasons such a limit (infinite sample averages) can never be reached. Thus, in this work we present the mean value of the measured variable with the $99.7 \%$ confident interval as $\xi_{c}=\left\langle\xi_{c}\right\rangle \pm 3 \sigma$. Similar analysis was also done for the estimates of $\gamma_{M C}, \kappa_{M C}$ from Eq. 8 and percentage deviation from the mean value is presented in the results.

\subsection{Results}

\section{Part 1: case study}

Table 5.1: Parameters of the default L/L system analyzed using MC-SCF.

\begin{tabular}{lll}
\hline & Value & Units \\
\hline Box size & $200 \times 200 \times 30$ & {$[b \times b \times b]^{1}$} \\
$t_{\text {eq }}$ & 24 & MCS $^{2}$ \\
$\Delta_{x}=\Delta_{y}$ & 5 & {$[b]$} \\
Simulation time & 125000 & MCS \\
Samples & 5000 & MCS \\
Acceptance rate & $41.45 \%$ & \\
Real time & 84 & days \\
Processor & Intel Core i7-2600 & \\
Clock speed & 3.4 & $\mathrm{GHz}$ \\
Cores, Threads & 4,8 & \\
& &
\end{tabular}

\begin{tabular}{lll} 
Chain length & 4 & {$[b]$} \\
$\chi_{c r}$ & 0.51 & \\
$\chi^{c r}$ & 0.50 & \\
$\gamma_{p}$ & $1.20 \times 10^{-3}$ & {$\left[k_{B} T b^{-2}\right]$} \\
$\kappa$ & $2.04 \times 10^{-2}$ & {$\left[k_{B} T\right]$} \\
$\xi$ & 4.124 & $b$ \\
$\gamma_{M C}$ & $3 \times 10^{-3} \pm 17 \%$ & {$\left[k_{B} T b^{-2}\right]$} \\
$\kappa_{M C}$ & $3 \times 10^{-1} \pm 29 \%$ & {$\left[k_{B} T\right]$} \\
$\xi_{c}$ & $7.48 \pm 3.69$ & {$[b]$} \\
\hline
\end{tabular}

\footnotetext{
${ }^{1}$ Here $b$ denotes size of a lattice unit (segment)

${ }^{2}$ Monte Carlo steps
} 
Let's take as an example two molecular components, namely $A_{4}$ and $B_{4}(N=4)$ This system has a critical $\chi^{c r}=0.5$ and a critical density $\varphi_{A}^{c r}=\varphi_{B}^{c r}=0.5$. The default value of the interaction parameter is $\chi=0.51$. For this case, the rigidity parameters are established in our earlier work (without the pinning constraints) [134]. Interfacial tension $\gamma_{p} \approx 1.2 \times 10^{-3}$ (units $k_{B} T / b^{2}$ ) and the mean bending modulus is $\kappa \approx 2 \times 10^{-2}$ (units $k_{B} T$. The cross-over length $\xi=4.124$ (units $b$ )). The latter value is found to be independent of the interaction parameter.

The chain length $N=4$ was considered the best choice, where both bending and tension dominated regimes can be observed in the structure factor, with least possible computational time. Choosing a slightly higher $N$ will have led to an increase in the computational time and complicated the analysis as $\xi$ would have become slightly dependent on $\chi[134]$. Choosing a slightly lower $N$ might have been possible. In which case, for a same $\Delta \chi=\chi-\chi^{c r}$, the $\gamma$ and $\kappa$ will be lower. Eventually leading to a longer equilibration time (and thus the simulation time). To adjust for it, $\Delta \chi$ could have been increased. Thus, a choice of $N=4$ and $\Delta \chi=0.01$ remained a reasonable choice. The system size was taken to be 200 lattice sites in both $x$ and $y$ directions and 30 sites in the $z$-direction. The latter was large enough to prevent the interface from approaching the upper or lower boundary of the system.

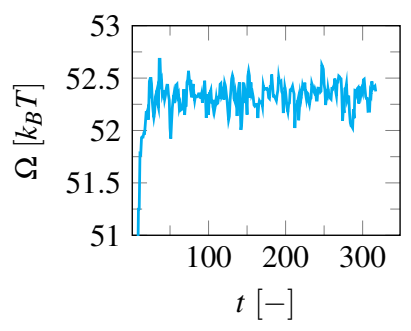

Figure 5.1: Variation of grand potential $\Omega$ as a function of Monte Carlo steps $(t)$ for the default system. Parameters of the default system are provided in Table I.

For this case, several trial MC-SCF calculations were initially performed to determine the $t_{e q}$, cf. Fig. 5.1. The grand potential of the system is presented as a function of the Monte Carlo time $(t)$ for one of the trial runs performed. It can be observed that after a few moves the interface reaches an equilibrium. For the default case, the average $t_{e q}$ was estimated to be 24 MCS. Note, providing a correlated move only fastens the equilibration time. Finally, a production run with 125000 Monte Carlo steps was performed and 5000 uncorrelated samples were collected (samples were assumed to be uncorrelated after every $t=t_{e q}$ steps). For details look at Table I. The simulation time (in days), though doesn't paint a rosy picture, 
could be easily factored up by 8-10 times upon parallelization.

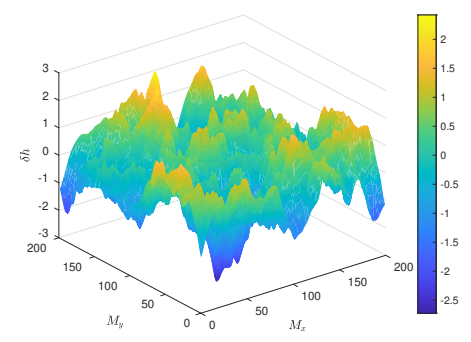

Figure 5.2: Analysis of simulated interface. Gibbs plane of the accepted interfaces are sampled and analyzed. Height fluctuations away from the mean Gibbs plane is determined at every $(x, y)$ lattice site and used to compute the fluctuation spectrum.

For each of the collected un-correlated sample tracking the interface provides $h(x, y, t)$ as explained before. In Fig. 5.2 an instantaneous snapshot of $\delta h(x, y, t)=$ $h(x, y, t)-\langle h(x, y, t)\rangle$ is presented in the normalized coordinates. It is rather counterintuitive to visualize the deviations to have high-amplitude at smaller wavelength and low-amplitude at larger wavelengths, as can be seen. However such a result is anticipated from the area penalty. Larger wavelength deviations cover a bigger area and the associated free energy increases dramatically even for a very small amplitude. The converse happens for smaller wavelength undulations.

In Fig. 5.3 contours of mean (left), square of mean (middle) and Gaussian curvature (right) are presented. At small scales it can be observed there are regions of positive and negative curvatures. A continuous region of positive curvature is often counteracted with a continuous region of negative curvatures. Such counter values of $J$ and $K$ enables the system to minimize the effect of curvature energy due to the $\mathcal{J}$ and $\mathcal{K}$ terms. This is naturally enforced by moving the pins as coordinated waves and having similar amounts of the two liquids. We will quantify these terms in the next paragraph. However, the physical significance of these terms is crucial to understand the fluctuations. Meanwhile, the contributions from $\mathcal{H}$ are always, unsurprisingly, positive. In an attempt to minimize this contribution, the system has to avoid high curvatures (both strong positive $J$ and negative $J$ ). Mere existence of mean and Gaussian curvature at small scales indicate that they contribute to the overall grand potential. The mean curvature integral is forced to remain zero by enforcing similar amounts of two liquids (not strictly enforced). This is not the case for the integral of the mean curvature squared. The growth of this contribution is damped by the mean bending modulus $\kappa$. In the same token the integral of Gaussian curvature is purely controlled by $\bar{\kappa}$. Thus, a positive high 

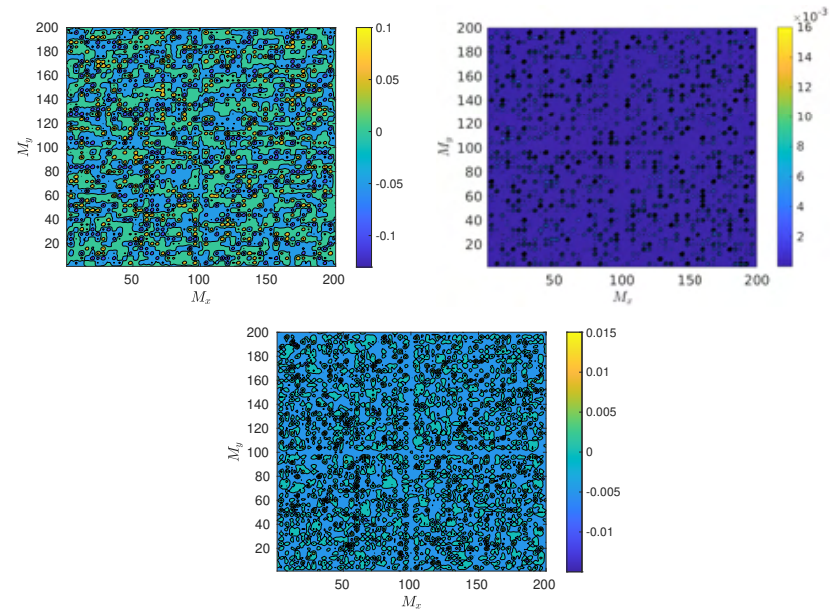

Figure 5.3: Contours of mean (left), square of mean (middle) and Gaussian curvature (right) at the traced interfacial points [in units of $b^{-2}$ ]. Because of local undulations both mean and Gaussian curvature feature at the interfaces. As a result every Mote Carlo move or a sampled mode changes the interfacial area, mean curvature and the Gaussian curvature. Thus it is impossible to say that the undulations are damped just by $\gamma, \kappa$. Among other higher order terms $\bar{\kappa}$ will play a vital role.

$\bar{\kappa}$ will attempt to reduce the Gaussian curvature. Eventually, this feature will show up in our estimated correlation length $\xi$. System with near zero $\bar{\kappa}$ will allow the interfaces to freely develop Gaussian curvature (preferring more saddle like undulations compared to the case that $\bar{\kappa}<0$ but obviously less when $\bar{\kappa}>0$ ).

In Fig. 5.4 the integrals of the area, mean curvature, square of mean curvature and the Gaussian curvature are presented. Using the known values for $\gamma, \kappa$ and $\bar{\kappa}$ we can (re) compute the grand potential of the system and compare it to the value that is computed directly from the SCF result of the 3D system. It turns out there is a close match between the two values. Hence the grand potential recovered from the integrals, $\Omega=\gamma \mathcal{A}-\kappa J_{0} \mathcal{J}+\frac{1}{2} \kappa \mathcal{H}+\bar{\kappa} \mathcal{K}$ matches that of the SCF-result. From the reconstruction we see that the major contribution arises from the interfacial area increase. Average value of the integral of mean curvature reaches zero as expected. Second largest contribution arises from the $\mathcal{H}$. Third contribution arises from the integral of the Gaussian curvature. The 'bookkeeping' is accurate up to $10^{-3}$ proving that the evaluation of the area and the curvatures is fairly accurate and that the thermodynamical and mechanical parameters derived previously[134] for this system are indeed contributing to the grand potential of the undulating interface. 

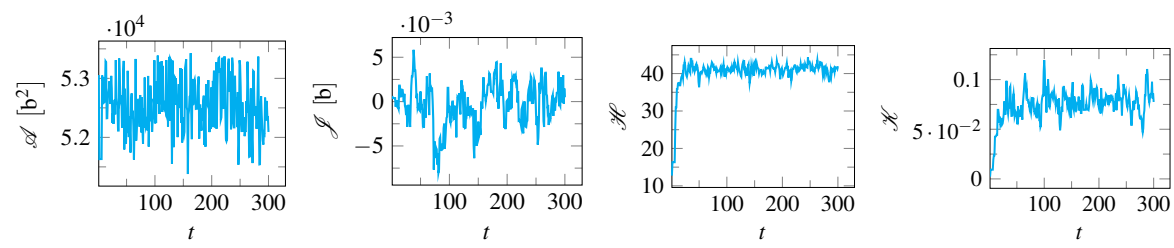

Figure 5.4: (a) Interfacial area $\mathcal{A}$ evolution as a function of Monte Carlo time steps. (b), (c), (d) represents the $\mathcal{J}=\int J d A, \mathcal{H}=\int J^{2} d A, \mathcal{K}=\int K d A$.

In Fig. 5. the probability density function of the interfacial height fluctuations is shown. This is a crucial yet intuitive result. In the MC-SCF, the metropolis algorithm ensures that the proper Boltzmann distributions of the system are sampled. Incremental energy in the grand potential $\Delta \Omega$ because of a small undulation $\delta h$ can be modelled as $\Delta \Omega=f(\delta h)$. Thus, the undulations should also feature a Boltzmann (Normal) distribution, as seen. Above statement is provided to provoke the reader's intuition and a strong mathematical proof is out-of-scope of the current work. It should be noted, that interfacial fluctuations are shown to be Gaussian elsewhere as well for gravity-dominated systems[177-179]. Several theoretical works, rather, assume such distribution. In MC-SCF, a Gaussian distribution occurs as an effect and not taken as an input condition.

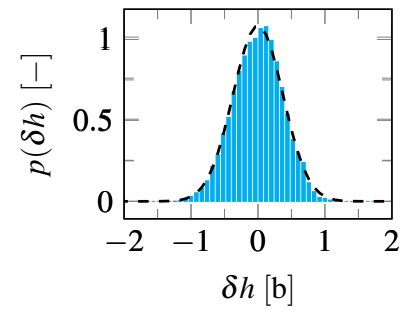

Figure 5.5: Probability density function, $p(\delta h)$, of interfacial height fluctuations of an instantaneous MC step. Note, $\int p(\delta h)=1$. A Boltzmann-like (Gaussian) distribution for interfacial undulations is observed. Dashed line is a Gaussian fit with a mean $\mu=0$ and a standard deviation of height fluctuations $\sqrt{w^{2}}=0.373$ [in units of $b]$.

Two-point correlation is presented in Fig. 5.6. Earlier it was mentioned that for self-affine surfaces the two-point correlation function follows Eq. 6. If the undulations are perfectly correlated the value of $G(\delta r)$ becomes 0 . As the distance between the points increases, the correlation exponentially decays. In this regime 
$G(\delta r)$ follows a slope of $2 H$ ( $H$ is the Hurst exponent) in log-log coordinates. It can be seen from the figure, that for the presented case $H=1$.

For interfaces that feature Gaussian undulations, as see in Fig. 5.5, are known to have an Hurst exponent $H=1$ [175]. Further, when the distance between the points equals the correlation length $\delta r=\xi_{c}$, the two-point correlation saturates to a value of $2 w^{2}$ (twice the variance of the distribution of undulations). From the $G(\delta r)$, the value of variance is estimated as $w^{2}=0.21$. For the presented case study, the mean correlation length $\left\langle\xi_{c}\right\rangle$ was found to be 7.48 by fitting the estimated $G(\delta r)$ with Eq. 6. R-squared value of the fit was 0.98 .

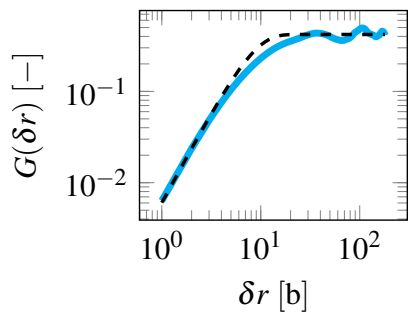

Figure 5.6: $G(\delta r)$ as a function of $\delta r$. The radial distribution function of the height fluctuations provide with an estimate of $\xi_{c}$. For the presented simulations the estimated value of $\xi_{c}=7.48$ by fitting $G(\delta r)$ with Eq. $6 . R^{2}$ for the fit was 0.98

In Fig. 5.7, the structure factor for the case study is shown. This hints at the presence of a the flow of energy in the system. Elsewhere in the field of physics, that is Turbulence, the transfer of energy from large scale structures to smaller scale structures occurs with a slope of $-5 / 3[180,181]$. Such a process is often referred to as an energy cascade. In interfacial systems, converse, Energy ascension happens. Energy builds up from small scale structures to larger scale. (high $q$ to low $q$ ). This is crucial, as it explains the difficulty in obtaining the spectrum using molecular level simulations. In simulations one has to wait until the energy builds from the molecular length scale to the mesoscopic lengths. Closer to critical points, such energy ascension is unhindered because of smaller rigidity parameters leading to a faster transfer. Eventually, the system reaches a homogeneous state (equilibrium). Within the MC-SCF framework, we can not observe or quantify this energy ascension. In the future, a dynamic variant of MC-SCF or molecular dynamics simulations can shed more light.

As it is clear that the structure factor has the information about the rigidity parameters, in Fig. 5.8, we present $1 / S(q) q^{2}$ as a function of $q^{2}$, where the intercept provides an estimate for interfacial tension and the slope of the linear regression provides an estimate for mean bending rigidity. The dashed line is a linear regression fit for the data. For the presented case, an estimate of $\gamma_{M C}=3 \times 10^{-3}$ 


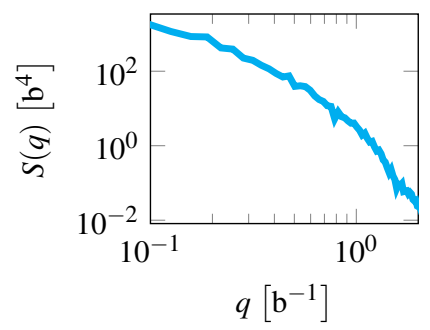

Figure 5.7: Fourier spectrum from the interfacial height fluctuation. Fluctuation modes are coupled as introduced by Meunier[182] and the energy builds up from higher $q$ to lower $q$.

and $\kappa_{M C}=3 \times 10^{-1}$ was obtained. It should be noted that both $\gamma_{M C}$ and $\kappa_{M C}$ are not too far off with its weak curvature counterpart $\gamma_{p}$ and $\kappa$ determined using SF-SCF (and mentioned above). Moreover, It is possible to carefully choose a range of $q^{2}$ that brings the $\kappa_{M C}$ closer to $\kappa$ at weak curvature limit. Simulations, that do not have the information from mean-field theories cannot have an overview of this range (except they could choose a regime where the points are more or less linear). This will eventually add to the error on the estimate of the rigidities (and this affects $\kappa_{M C}$ estimate more than the $\gamma_{M C}$.

Above obscurity arises as a consequence of the numerical noise associated with the discrete lattice in the simulations (For example, a spike in the data, at $q^{2}=0.6$ ). Discrete lattice aggravates the errors and prevents a closer comparison of the rigidities to their mean-field counterpart. Additionally, $\xi_{c}$ can also be obtained (approximately) from the structure factor based estimates of rigidity parameters as $\xi_{c} \approx \sqrt{\kappa_{M C} / \gamma_{M C}}=10 \pm 23$. Not unsurprisingly, the value is a factor of 2 (and has an enormous error bound) off from the expected weak curvature estimate from SF-SCF. Thus, in the rest of the work, we will use the $G(\delta r)$ to estimate the correlation length, as it is a better measure that avoids the error from linear regression fitting. The above results, clearly indicate that the results obtained from the structure factor are prone to errors from lattice discretization and numerical fitting. Further, the omission of the presence of a finite $\bar{\kappa} \mathcal{K}$ in the above estimates adds to the errors.

\section{Part 2: Parameter variations}

From the case study, it was clear that the MC-SCF provides an reasonable representation of the interfacial fluctuations. It was also clear that the estimation of the rigidity parameters will always be erroneous because of the linear fit over an obscurely defined range. However, it was shown that the correlation length $\xi_{c}$ 


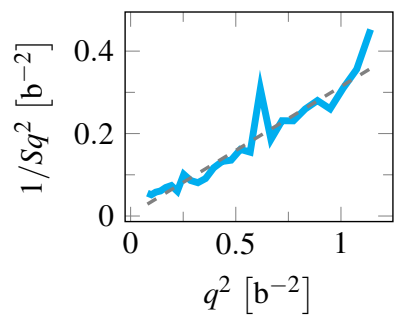

Figure 5.8: Estimation of tension and bending rigidity through linear regression. For the presented case $\chi=0.51, N=4$. Equation of the regression line is $y=0.003+0.3 x$ corresponding to $1 / S q^{2}=\gamma+\kappa q^{2}$.

estimates were still meaningful despite the significant error bars.

Table 5.2: Results of constant tension simulations, $\gamma_{p}=0.01 k_{B} T b^{-2}$

\begin{tabular}{llll}
\hline & Case 1 & Case 2 & Case 3 \\
\hline Box size & $100 \times 100 \times 30$ & $100 \times 100 \times 30$ & $100 \times 100 \times 30$ \\
$\mathrm{~N}$ & 200 & 20 & 4 \\
$\Delta \chi$ & 0.007 & 0.03 & 0.09 \\
$\Delta_{x}=\Delta_{y}$ & 11 & 13 & 5 \\
$\kappa\left[k_{B} T\right]$ & 1 & 1.5 & 0.1 \\
$\bar{\kappa}\left[k_{B} T\right]$ & 0.56 & 0.79 & 0.048 \\
$\xi$ & 10 & 12.24 & 3.16 \\
$q^{*}(\pi / \xi)$ & 0.31 & 0.25 & 0.97 \\
$\left\langle q_{c}^{*}\right\rangle$ & 0.61 & 0.41 & 0.92 \\
$\xi_{c}$ & $5.14 \pm 4.09$ & $7.65 \pm 3.89$ & $3.41 \pm 1.23$ \\
\hline
\end{tabular}

This motivated us to perform a series of simulations for systems that have, at specified $\chi, N$, a constant estimate for the interfacial tension $\gamma_{p}$, yet different values for the mean bending modulus $\kappa$ and the other way around. These estimates follow from our previous article[134]. Parameters of such calculations are provided in the Table 2 and Table 3.

In Fig. 5.9, we present the structure factor for all the simulated cases. As shown, in our case study, it is indeed difficult to exactly reproduce the rigidity parameters. However, we see that the major trends do follow the expectations. In the constant $\gamma$ simulations, the structure factor converge at the low wavenumber limit. In the constant $\kappa$ simulations, the structure factor converge at the high wavenumber limit.

All our simulations are chosen to lie closer to $\bar{\kappa} \approx 0$ point. When this happens, it can be expected that the $\xi$ calculated from the mean-field parameters should be close to the observed value from the simulation. Indeed, we do see this trend for our 
5. Imposing height undulations on the liquid-liquid interface

Table 5.3: Results of constant rigidity simulations, $\kappa=0.9 k_{B} T, \bar{\kappa} \approx 0.45 k_{B} T$

\begin{tabular}{llll}
\hline & Case 4 & Case 5 & Case 6 \\
\hline Box size & $100 \times 100 \times 30$ & $100 \times 100 \times 30$ & $100 \times 100 \times 30$ \\
$\mathrm{~N}$ & 200 & 20 & 4 \\
$\Delta \chi$ & 0.003 & 0.02 & 0.2 \\
$\Delta_{x}=\Delta_{y}$ & 13 & 11 & 5 \\
$\gamma\left[k_{B} T b^{-2}\right]$ & 0.006 & 0.008 & 0.05 \\
$\xi$ & 12.25 & 10.6 & 4.24 \\
$q^{*}(\pi / \xi)$ & 0.26 & 0.30 & 0.74 \\
$\left\langle q_{c}^{*}\right\rangle$ & 0.21 & 0.42 & 1.10 \\
$\xi_{c}$ & $14.95 \pm 3.43$ & $7.47 \pm 5.23$ & $2.85 \pm 1.06$ \\
\hline
\end{tabular}

simulations. Further as the $\bar{\kappa} \approx 0$, for case 3 , we observe that the observed $\xi$ from simulations lie closer to the expected mean-field result. However, the error bars observed in the calculation prevents us from exploring the role played by $\bar{\kappa}$ in detail. Though a detailed comparison is not possible, it can be said with certainty that the $\bar{\kappa}$ plays a role in the fluctuating interfaces as seen in a periodic boundary simulation box. As said before, this occurs to compensate and correct for the fluctuations in the geodesic curvature while maintaining a constant Euler characteristic. Thus, we cautiously note, for an infinitely large or a closed interface the Gaussian bending rigidity will not influence the fluctuations. Further, it can be seen from the Tab. 2. and the Fig. 9 (left) that at a fixed $\gamma$ the match (comparatively) of trends in $\xi_{c}$ from simulations with $\xi$ from mean field calculations means a trend in $\kappa$ is recovered as expected. Similary, from Tab. 3. and Fig. 9 (right), it can be seen that the trends of $\gamma$ is recovered as expected.
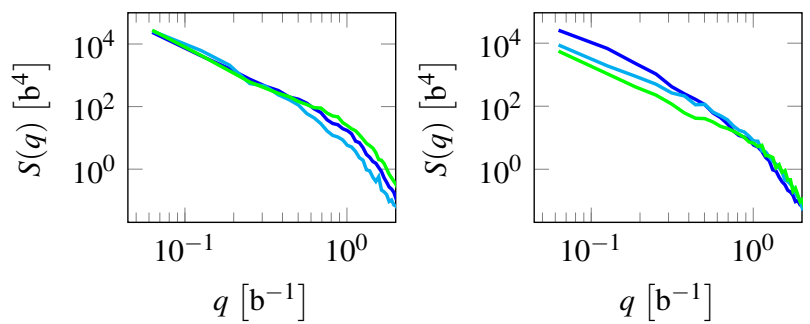

Figure 5.9: Spectrum of height fluctuations of constant tension and constant mean bending rigidity simulations. (Left: blue, cyan, green correspond to $N=200,20,4$ and $\Delta \chi=0.007,0.03,0.09$ respectively where $\kappa=1,1.5,0.1, \gamma=0.01$ (cf table 5.2) Right: blue, cyan, green correspond to $N=200,20,4$ and $\Delta \chi=0.003,0.02$, 0.2 when $\kappa=0.9, \gamma=0.006,0.008,0.05$ (cf table 5.3) 


\subsection{Discussion}

We focused on a purely symmetric system for which the weak curvature values of rigidity parameters were well established [134]. Our target is to compare the estimated weak curvature rigidity parameters to that of strong curvature limit when interfaces fluctuate. Introducing pinning points in the SF-SCF formalism made it possible to implement an MC-SCF hybrid simulation that can simulate a fluctuating symmetric interface. Idea was to analyze such simulated interfaces to achieve our target of comparing the rigidity estimates in two limits. Furthermore, computationally, a periodic boundary condition remained the only option to simulate such interfaces. However, the above choices contributed to two important factors: (i) pinning introduced a discreteness in the fluctuation modes (ii) periodic boundary caused a change in geodesic curvature, in turn, introducing a change in Gaussian curvature. In the following paragraphs, we will discuss how these factors affect the estimates of $\gamma$ and $\kappa$ of fluctuating interfaces.

Let's discuss the discreteness issue firstly and later we will discuss the effects of fluctuating Gaussian curvature. In MC-SCF hybrid, the pinning points are MC degrees of freedom. The 3-gradient SCF model, in turn, takes these pinning points as constraints to find the interface that follows these pinning points. The calculations are done in the grand canonical ensemble, that is, the bulk concentration of the two solvents is fixed to the binodal value. The characteristic function is the grand potential and this quantity serves as the internal energy in the Metropolis algorithm of the MC simulation. The SF-SCF formalism is lattice-based and as a result, the pinning points can only be placed a few lattice sites apart so that the SCF solution can be swiftly found. This implies that on a smaller scale the fluctuations are averaged out in a mean-field fashion. In other words, the small scale resolution of the fluctuations is out of the scope of the MC-SCF method. At the current state of the art, we cannot resolve undulation fluctuations on the scale smaller than or similar to the lattice site. The adverse effects of it were seen in the $S(q)$ plots which do not show the correct result at high values of $q$. This is troublesome because exactly in this range the important questions regarding the role of $\kappa$ in the height fluctuations reside.

To improve the resolution for shorter wavelengths. We might need to increase the number of pinning points in the system, but for this, the lattice should be less coarse. The approach calls for a significant refinement of the lattice. In SF-SCF the grid size (lattice site length) is set equal to the segment size. One can envision (a future) doubling of the precision in space by taking the grid size twice smaller than the segment size, or three times, etcetera. In 3D systems, this will increase the 'system size' by a factor of 8 , or 27 , etcetera. Although the 8 times increase might still be in reach, a significantly finer grid would not be feasible soon.

Now, it is time to understand the effect of fluctuations in Gaussian curvature in 
a periodically bounded simulation box. Above (cf. Fig. 5.4) we have seen that for a given snapshot we can evaluate the grand potential. After the interface position $h(x, y)$ is found, we were able to find the contribution to the area $\gamma \int d A$ as well as that from the mean curvature squared $\frac{1}{2} \kappa \mathcal{J}$. We found it is necessary to also use the contribution of the Gaussian curvature $\bar{\kappa} \mathcal{K}$ to complete the bookkeeping. The latter quantity is not constant. We reconciled this by assuming that the line integral of $\int k_{g} d s$ along the edge of the interface in our periodic box 'compensates' for these fluctuations such that the Euler characteristic $\chi=1$, irrespective the shape of the interface. We reconciled this by assuming that the line integral of $\int k_{g} d s$ along the edge of the interface in our periodic box 'compensates' for these fluctuations such that the Euler characteristic $\chi=1$, irrespective the shape of the interface.

A natural consequence is that for finite-sized pieces of an interface the fluctuations are not only controlled by $\gamma$ and $\kappa$ but also by $\bar{\kappa}$. We know from previous works that binary liquids far from their critical point have a negative value for $\bar{\kappa}$. It is not impossible for simulations of such systems to feature a negative effective bending rigidity, i.e. when the saddle splay modulus (which is strongly negative) and the mean bending modulus (which invariably is positive) combine such that the former dominates. This might explain the simulation results of the Leiden team some years ago,[66] that found difficulty in finding convincingly the sign of the bending modulus from MD computer simulations.

Our results show that the grand potential of a snapshot can indeed be decomposed in an area, mean curvature square, and a Gaussian curvature contribution. This means one does not need to use an expensive MC-SCF hybrid to do computer simulations of a fluctuating interface. We can implement (as already done in the literature) a 'membrane' model[78, 183], wherein the interface is seen as 2dimensional constructs of which the shape can be changed and the corresponding change in the grand potential can be estimated, e.g. using triangulating the interface. Such 'membrane' simulations are an order of magnitude (or more) faster than the current approach and give the results that are now expected to match the ones we have done in this chapter.

To go beyond the mean-field variant of a fluctuating interface, we need to also include fluctuations in the two bulk phases that surround the interface. Indeed, in reality, bulk and surface fluctuations are expected to go hand in hand. We believe that work in this direction is of more than average interest as this might also lead to renormalized values for the mechanical parameters of the interface.

We know from SF-SCF results, for the microemulsion systems, that by changing the system parameters (chain length of the surfactant $N$ and that of the solvents $n$, and the interaction parameter $\chi$ ) it is possible to consider topology changes, e.g. the transition from a lamellar phase to a sponge phase. In the future, MC-SCF hybrid calculations can potentially become an effective tool to address such problems and consider simulations in which the topology of the interface is altered. 
The primary idea of the MC-SCF simulations, presented in this work, is to establish credibility. The method excludes bulk effects in the fluctuations, but apart from that the fluctuations appear to be accurately represented, that is, on a mean-field level.

\subsection{Conclusions}

We considered simple molecularly symmetric systems $\mathrm{A}_{N}-\mathrm{B}_{N}$ that have just a single interaction parameter $\chi_{A B}$ using regular solution-like modeling. A Monte Carlo self-consistent field (MC-SCF) hybrid simulation is presented which can excite a relevant interface with height undulations. The grand potential of such fluctuating interface $\Omega$ could be reconstructed from the estimated area, estimated square of the mean curvature, and the mean Gaussian curvature of the interface for a given snapshot, using independently obtained coefficients for the interfacial tension $\gamma$, the mean bending modulus $\kappa$ and the saddle splay (Gaussian bending modulus) $\bar{\kappa}$. To extract, in turn, these interfacial coefficients from the height fluctuations, turned out to be not extremely accurate because of loss of 'precision' and coarseness of the underlying lattice model.

Furthermore, by analyzing the individual snapshots, it was found that for a finite interface (within a periodic box), the fluctuations are controlled not only by $\gamma$ and $\kappa$ but also by $\bar{\kappa}$. The line integral of geodesic curvature compensates for the Gaussian curvature induced in the simulated interfaces. The presence of finite Gaussian curvature in our simulated interfaces may influence our rigidity estimates as well, adding an inaccuracy on top of the precision loss due to discreteness.

In future works, eliminating one of the two effects will shed more light on which of the above two factors is the primary reason for the inaccuracy caused in the estimation of the rigidity parameters. The source of inaccuracy, in estimating the rigidities from a fluctuating interface, is not explicitly clear from our current work. We leave this as an open research question. Nevertheless, our current results validate the approach followed by so-called membrane models to study fluctuating interfaces on mesoscopic length scales.

The current MC-SCF formalism is suitable to study interfacial fluctuations, albeit that the CPU time must be kept under control. To distribute the 'work' on a GPU may prove to be a necessary next step.

The MC-SCF hybrid approach is shown to be a credible way to model interfacial fluctuations of simple symmetric interfaces. We now envision that the method may also become of interest to probe the fluctuation effects in a wider context of the physics of interfaces. One natural extension is the addition of surfactants leading eventually to the formation of (micro)emulsions. Realistic future lines of research may focus on how fluctuations manifest in microemulsions wherefore the interfacial 
5. Imposing height undulations on the liquid-liquid interface

tension can become ultralow or how topological changes of interfaces may take place as a response to a sign change of $\bar{\kappa}$. 


\section{CHAPTER 6}

\section{Summary and General Discussion}

We started our journey with a desire to model microemulsions. In this chapter, we will discuss how close we are towards achieving our goal to model microemulsions. We started with a simple liquid-liquid interface. We analyzed its properties using a simple mean-field model. Later, we added surfactants to reach a tensionless state. We analyzed the properties of surfactant added interfaces. Once the fundamentals, accurate ways to quantify the interfaces, are established, we studied our models under different conditions. Finally, to include fluctuation information, we developed a Monte Carlo hybrid simulation technique. However, in all our works, we studied the ideal scenarios. Here, we will map the relevance of our results to microemulsions. The later part of this chapter will provide guidelines on some of the future research pathways that can be adapted. 
We started our journey with a desire to model microemulsions. An ideal oneparameter model was used throughout our thesis to understand the fundamentals of interfaces. Several crucial details were missing in the literature when we started our work. To highlight a few of those: 1) Estimation of rigidities that quantify the interfaces were either ambiguous or erroneous both for simple L/L interfaces and surfactant added L/L interfaces. 2) Fundamental insight behind the phase transitions in a three-component system was not available. 3) Simulations did not have a clear overview of the parameter spaces they were operating. The major part of this thesis focuses on fixing these fundamentals missing blocks. It is a first and necessary step towards modeling microemulsions. In the next section, we will summarize our results that set up the fundamentals required to model microemulsions.

\subsection{Summary of our work}

\section{Symmetric L/L interface: Fundamentals}

As mentioned earlier, an ideal representation of a microemulsion system should be a tensionless, balanced interface. The building step towards such an ideal model is to have an interface. Then balance this interface such that there is no asymmetry in the system. Finally, make this interface tensionless. By skipping this last step, we created a balanced Liquid - Liquid (L/L) interface. In Chapter 2, we focused on such symmetric L/L interfaces. We used equal amounts of the liquids to attain a balanced state. Note, these interfaces have finite tension, unlike the ideal model that represents microemulsions.

Such L/L interfaces were analyzed using SF-SCF in the $(\mu, V, T)$-ensemble. By curving the interfaces into a cylinder and sphere lead to accurate quantification of the curvature energies. By comparing the curvature energies with their planar counterpart allowed a direct estimation of the fundamental parameters: $\gamma, \kappa$, $\bar{\kappa}$. There are two ways to remain in a $(\mu, V, T)$-ensemble. First, by choosing a scale-invariant topology. Second, by using a Lagrange-parameter to specify the interfacial position. Specification of the interfacial location gives freedom to lookup for a solution with a specific $\mu$. Inherently, this means we have to use the $\mu$ from the ground state while determining the curvature expenses in cylindrical and spherical geometry. A vital detail is the inclusion of near-neighbor interactions. Earlier, a similar approach by Matsen[35] performed a similar analysis for infinitely long polymer melts without including the Cahn-Hilliard like gradient density. By ensuring equal amounts, including near-neighbor interactions, remaining in Grand canonical ensemble, and acquiring solutions with high accuracy led to physically reliable results. Importantly, we argued that the exclusion of any of the above leads to errors. Below, we will summarize our results. Finally, we will map its relevance 
to reality and expose the rooms for improvements.

As stated above, we accurately estimated all the fundamental properties of symmetric L/L interfaces: Interfacial tension $(\gamma)$, mean bending rigidity $(\kappa)$, Gaussian bending rigidity $(\bar{\kappa})$. We found positive values for both the moduli when the system is closer to the critical point. Further, the rigidity parameters showed the same scaling as that of the interfacial tension. The role played by these parameters in thermal fluctuations were crudely reported [66, 184-186]. Earlier results show two regimes: At smaller length scales, $\kappa$ dampens the thermal undulations, and $\gamma$ dampens the fluctuations at larger length scales. By defining this length scale as a cross over length $\xi=\sqrt{\kappa / \gamma}$, we showed that $\xi$ is relatively constant and often remained at the size of a few segments. However, a non-monotonous behavior is observed in cross over length as a function of the temperature scale, featuring a maximum. More importantly, for larger chains, a significantly larger $\xi$ was observed. Finally, we also report a sign-switch of $\bar{\kappa}$. We speculated that the sign-switch happened when the width of the interface becomes smaller than a segment size.

Let's map the results of our model against experimental systems. Our model discussed above assumes symmetry. In reality, such symmetry is often impossible to achieve without the addition of a third component (co-solvent). Note, polymer melts with nearly equal molecular weight do come closer to our model system. However, typical oil and water mixtures are not as simple as the model discussed. Importantly, breaking the symmetry brings in an additional parameter into consideration. Spontaneous curvature $\left(J_{0}\right.$, a measure of Tolman length $\left.[48,187,188]\right)$ features in asymmetric systems. $J_{0}$ adds to the list of fundamental parameters. Another assumption that is implicit in the mean-field models is the averaged fluctuation and loss of co-operative movements of molecules. Both asymmetry and undulations are required to model reality $[189,190]$. We will further discuss these assumptions in the later sections, but here, we only stress that there is room for improvement.

\section{Tensionless balanced L/L interface: Fundamentals}

To make one step closer towards modeling microemulsions, in Chapter 3, we added surfactants to make the symmetric L/L interfaces. Surfactants partition at the interface between the liquids. The interface was made tensionless by iteratively adding more surfactants to the symmetric L/L interface. Simultaneously the amount of the solvents was adjusted, every step, to keep the system in a balanced state. While the head group of the surfactants were made from segments that made the first solvent, the tail group segments were made from segments that made the second solvent. This tensionless, balanced surfactant loaded liquid/liquid interface $(\mathrm{L} / \mathrm{S} / \mathrm{L})$ was introduced as an ideal model for microemulsions.

Indeed, a microemulsion of crude oil, water, and a surfactant or several other real-world applications are never perfectly tensionless or balanced. However, to 
understand such complicated mixtures it is essential to understand the ideal model and build upon the results. Thus, in Chapter 3, we discussed the accurate way to estimate the bending rigidities for the ideal model. Unlike the $\mathrm{L} / \mathrm{L}$ interfaces, studied in Chapter 2, the bending rigidities control the behavior of the tensionless interfaces. It was thus essential to estimate these parameters in a more reliable and reproducible way. Scale-invariant topologies provided an elegant route to estimate the rigidities: A minimal Torus gave a direct estimate of $\kappa$, The im $3 \mathrm{~m}$ cubic phase provided a direct-estimate of $\bar{\kappa}$. These scale-invariant geometries route to estimate the parameters were computationally intensive. An indirect route was established using the planar, cylindrical, and spherically curved interfaces to determine $\kappa, \bar{\kappa}$. By comparing the values with the direct estimates, the internal consistency of the indirect route was established.

Among several interesting results that were recovered from this model, a sign switch of the $\bar{\kappa}$ was found. Closer to the critical point $\bar{\kappa}$ was positive and at strong segregation far from the critical point $\bar{\kappa}<0$. It was well-known that for tensionless interfaces that $\kappa$ controls the fluctuation of the interfaces whereas $\bar{\kappa}$ controls the topology. A sign switch in $\bar{\kappa}$ was essentially argued to be the reason for phase transitions from lamellar to sponge-like microemulsions phases. It should be noted that similar trends were also observed in the simple $\mathrm{L} / \mathrm{L}$ interface, discussed in Chapter 2. However, for an interface with finite tension $\gamma>0$, the sign-switch though important was not entirely sufficient to drive topological changes. In both cases, the sign-switch was observed when the width of the interface exceeds the size of a segment. Finally, $\kappa$ remained positive irrespective of the chain length and the interaction parameter.

\section{Ternary system: Phase diagram}

In Chapter 4, we further analyzed the three-component systems discussed in Chapter 3. An advantage of the ideal model discussed in Chapter 3 is that upon gradually removing the surfactants and remaining in a balanced state the system will eventually reach the pure L/L system discussed in Chapter 2. Similarly, decreasing the amount of solvent will lead to the well-known microphase segregation limit. The phase diagram, in $\left(\chi, \varphi_{s}^{b}\right)$-coordinates, for such ternary systems, is known to feature two lines of critical points, Scott- and Leibler line. In Chapter 4, we showed that the results discussed in Chapter 3 features as a saturation line in the phase diagram. Further, the limiting case where $\varphi_{s}^{b}=0$ represents the system discussed in Chapter 2 (pure L/L interface).

The saturation line was shown to end at a special point, referred to as the Microemulsion point (MP). The $\kappa$ and $\bar{\kappa}$, relevant for the shape fluctuations and the topology of interfaces, respectively, are evaluated along the saturation line. Near the MP both rigidities are shown to be positive and vanish in a power-law fashion 
with coefficient unity at the MP. Overseeing these results proves that the MP has a pivoting role in the physics of microemulsions. We argued that it was essential to remain in the vicinity of the MP to form bi-continuous microemulsion sponge phases.

Essentially Chapter 4 thus discusses the connection between the two systems $(\mathrm{L} / \mathrm{L}$ and $\mathrm{L} / \mathrm{S} / \mathrm{L})$ discussed before in a crude sense. Earlier works have discussed a line of critical points upon adding surfactants to the pure L/L interface called Scott line. The endpoint of the Scott line was referred to as the Scott point(SP). Similarly, a line of critical points upon reducing the solvents in a microphase segregated ternary system is called the Leibler line. The endpoint of the Leibler line was referred to as the Leiber point (LP). In Chapter 4, we showed that the Scott line does not meet the Leibler line for real systems. We further showed that there exists a third axis to this phase diagram, namely the distance between the interfaces $D$. Upon considering this third dimension, it was found that there exists a region between the discussed MP, LP, SP. We referred to this region as the supercritical triangle. It should be noted that the earlier works that ignored the third parameter as they were focussing on infinitely long polymer chains found that MP, LP, SP all merged at a Lifshitz point. Finally, we showed that a pair of binodal lines that envelop the three-phase (Winsor III) microemulsion region, is shown to connect to MP. The cohesiveness of the middle phase in Winsor III was found to be related to the non-monotonic, inverse DLVO type interaction curves between the surfactant-loaded tensionless interfaces.

It is again time to do the check for reality. As always we ignored asymmetry and fluctuations that are essential for real systems closer to critical points. Several crucially important critical questions arose: 1) What happens to the topology of the interfaces closer to the MP? 2) What role do the thermal fluctuations play closer to the MP? 3) Does the fundamental parameters $\kappa, \bar{\kappa}$ estimated at the weak segregation limits remain valid in the strong curvature limits of undulating interfaces? 4) What happens to the rigidity parameters at every point in the phase diagram (our results only have data on the saturation line and the $\mathrm{L} / \mathrm{L}$ interface)?

\section{Interfacial Fluctuations: Fundamentals}

In Chapter 5, we attempted to address the fundamentals of interfacial fluctuations. More specifically, our search was to extract the rigidity parameter from a fluctuating interface and compare it with the weak curvature limit estimates for the $\mathrm{L} / \mathrm{L}$ system discussed in Chapter 2. A hybrid Monte Carlo SF-SCF method was developed (MC-SCF). Coordinated wave-like Monte Carlo moves were performed on the planar $3 \mathrm{D}$ interface. Interfaces were later analyzed.

In Chapter 5, we confirm that individual contributions from the undulations: increase in interfacial area, changes in the integral of the mean curvature, and 
the integral of the Gaussian curvature contribute to the overall change in the Grand potential. Essentially, this states that the weak curvature estimates of the fundamental parameters do govern the fluctuations of the interface (even in the high curvature limit). This is a crucial finding as the results from Chapter 5 sufficiently proves that fluctuations in any system can be modeled if $\gamma, \kappa, \bar{\kappa}$ are known.

We reasoned that the discreteness and finite system size in our simulations hindered a detailed exploration of the interfacial fluctuations. A discrete lattice prevented moves in a continuous fashion leading to signal aliases. Finite system size and segment size limited the range of waves that can be captured in the simulations. We note that even though the range captured in MC-SCF is better than many of the full atomistic or coarse-grained simulations [99, 191-194] could still be improved.

Interfacial fluctuations result from a dynamic process. A Monte Carlo simulation the fluctuations do not capture the dynamics of such fluctuations. This is an inherent problem with all Monte Carlo simulations. Further, in real systems, fluctuations exist all throughout the system and not just in the interface as modeled in Chapter 5. Such bulk fluctuations were argued to be the source of the interfacial fluctuations. However, a hard proof for such a statement remains to be explored from fundamental simulations. Finally, there are several works that argue an existence of scale-dependent fundamental parameters [195-197]. This paragraph showcases just a few of the unsolved questions in the field of interfacial fluctuations.

\section{Fundamentals are set}

Exploring ideal models using SF-SCF and MC-SCF we have so far set the fundamentals required to model generic microemulsion systems. We have removed some of the long-existed ambiguity in the field by carefully exploring very simple models. It should be mentioned that even such simple models proved to be extremely rich and featured complicated physics: A microemulsion point, threephase coexistence, vanishing rigidities, topological switches, etc. to mention a few. However, we have to move towards reality, as we discussed above, towards systems that are asymmetric and feature fluctuations. In the following section, we will explore some of the promising future research lines that would significantly add to our work.

\subsection{Microemulsion modeling: Future}

\section{Asymmetric L/L interface:}

As reviewed several times above, reality features asymmetric molecules and unequal amounts of liquids. Several works exist that attempt to determine the phases of a ternary system provided the input parameters $\gamma, J_{0}, \kappa, \bar{\kappa}$ [198-200]. Amazingly, 
several such models do predict an expected fish-like phase diagram [45]. This brings up an interesting question, can we predict the phase of a ternary mixture from just knowing the composition and molecular architecture? Answer to such a question comes in two steps: 1) Predicting the fundamental parameters $\gamma, J_{0}, \kappa, \bar{\kappa}$ provided the molecular composition, temperature, and amounts. 2) From the fundamental parameters, determining the phase behavior using a hybrid simulation that accounts for thermal fluctuations accurately. Both the steps are essential, simulations that often ignore the first step often operate in unknown territory and attempts to explain the observed phases. Models that ignore the second step often speculate the phases that can exist for the given fundamental parameters.

Our work laid the foundations to do precisely that. We laid the groundwork to estimate the fundamental parameters. Further, it proved that the fundamental parameters play a significant role in dampening the thermal fluctuations. However, much remains unknown when asymmetry comes into play. We have not established a reliable route to estimate the fundamental parameters of an asymmetric $\mathrm{L} / \mathrm{L}$ or L/S/L interface. Guerra et. al. attempted to estimate the parameters of an asymmetric ternary mixture [201]. The focus was given to study the behavior of approximate free energy density-functional for a wide range of interaction parameters. The work also reported the effective bending rigidity $2 \kappa+\bar{\kappa}$ in the presence of asymmetry. Vector models that model the three-component systems using a Hamiltonian have also been proposed [202, 203]. To our knowledge, much of the above-mentioned works only provide a partial picture: not all parameters $\left(\gamma, J_{0}, \kappa, \bar{\kappa}\right)$ were obtained unambiguously. Further, our MC-SCF only proved that $\gamma, \kappa, \bar{\kappa}$ are involved in dampening of the thermal fluctuations but did not provide a realistic picture of how these parameters will be modified in a fluctuating interface. It even remains unknown whether the fundamental parameters will be modified. We will discuss the effects of fluctuations in the later sections. In this section, we will discuss how to find the fundamental parameters if asymmetry features in our systems.

Asymmetry can come into play by several routes. In the following sections, we will discuss a few asymmetric systems that allow us to generalize our ideal models for both L/L and L/S/L systems. Firstly, let's take our simple L/L system made and generalize it for two liquids $A_{m}, B_{n}$. In Chapter 2, we modeled the special case, where $m=n$. This system still features one $\chi$ that dictates the interaction between the segments. Helfrich equation for such a system will include an additional term that contains the spontaneous curvature term,

$$
\gamma(J, K)=\gamma(0,0)-\kappa J_{0} J+\frac{1}{2} \kappa J^{2}+\bar{\kappa} K
$$

Two interesting routes can be followed for such a system: 1) Learning to live with asymmetry 2) Balancing the system by adding co-solvents, namely $A_{n}, B_{m}$ to counteract the existing asymmetry. Let's first discuss, how to evaluate the rigidities. 
As we have established that $\bar{\kappa}=\int-z^{2} p(z) d z$ can be roughly estimated even for an asymmetric interface from the planar ground state. Similarly, a rough estimate for $-\kappa J_{0}=\int z p(z)$ can be obtained by simply analyzing the planar interface [37]. While remaining in the $(\mu, V, T)$-ensemble, the remaining parameters can be obtained by curving the interface into a cylindrical and spherical geometry. Using a Lagrange parameter, as discussed in Chapter 2, makes such an analysis possible as $\mu$ can be fixed as that of the planar ground state. From the difference in grand potential of the cylindrically curved interface, $\Delta \Omega^{c}=-2 \pi \kappa J_{0}+\frac{\pi \kappa}{R}$ will allow to an estimate of $\kappa$ upon using the $\kappa J_{0}$ estimated using the first moment. Finally, the difference in the grand potential of a spherically curved interface $\Delta \Omega^{s}=4 \pi\left(-2 \kappa J_{0} R+2 \kappa+\bar{\kappa}\right)$ can be used to check the internal consistency. Further, a minimal torus and $\operatorname{Im} 3 \mathrm{~m}$ cubic phases remain to be an elegant route towards estimation of $\kappa$ and $\bar{\kappa}$ [204] as they do not feature a mean curvature integral in their curvature energy.

From the above equations, it is immediately visible that higher the $J_{0}$ more preferable it is to form cylindrical or spherical phases (micellar phases). Upon increasing the gap between $m$, and $n$ the asymmetry should increase the $J_{0}$. An interesting comparison will be to analyze the second route by adding co-solvents that would balance the asymmetry. For a given amount of $A_{m}, A_{n}, B_{n}$ the amount of $B_{n}$ can be optimized such that $J_{0}=0$. For such a mixture once the amounts of individual components are known, a Willmore torus and an Im3m cubic phase will provide a reliable estimate of $\kappa, \bar{\kappa}$. Sufficient fundamental insights from such generalization would allow us to develop a more generic model for the ternary mixtures. However, we note that even though the pathway is clear, computational difficulties may prevent such pathways. Lattice artifacts, discreteness of the lattice, energy minimization in complex geometries like Willmore Torus and Im3m cubic phases, isometric face generation in smoothly curved interfaces, are significant problems that have to be addressed before progress can be made.

\section{Asymmetric L/S/L interface:}

Asymmetry in surfacted liquid-liquid interfaces (L/S/L) can be introduced through several means: 1) Asymmetry in surfactant architecture 2) Asymmetry is solvent architecture 3) Asymmetry in the amount of solvents. In Chapter 3, 4, we analyzed an ideal balanced model $A_{n}, B_{n}, A_{N} B_{N}$. By modeling the surfactants as $A_{M} B_{N}$, interfaces will feature a non-zero spontaneous curvature as discussed above. The same applies to systems where the solvent composition is made different. ex. $A_{m}, B_{n}$, $A_{N} B_{N}$. Finally, the ideal system can be made asymmetric by gradually removing one of the solvents, say $B_{n}$. Such a system will eventually evolve into a bilayer membrane. A generic system does feature an amalgam of all such asymmetries. By analyzing systems that introduce asymmetry either through solvents or surfactants or amounts provides a step-wise way towards modeling such a generic system. The 
route to obtaining the fundamental parameters remains the same: Moments of planar geometry provide $\bar{\kappa},-\kappa J_{0}$, cylindrically and spherically curved interfaces provide an indirect estimate of $\kappa, \bar{\kappa}$. Willmore torus and $\mathrm{Im} 3 \mathrm{~m}$ cubic phase remain as a check to prove internal consistency.

\section{Application oriented modeling}

One step further will be to model a system that contains crude oil mixed with water. Crude oil is a mixture of naphthalenes, asphalts, aromatics, and paraffin. This will lead to a bulk system that does not have just two liquids, but rather a mixture of several liquids with different chain length (all phase separating from the water phase). A more complicated model will look like $N a_{w}, A s_{x}, A r_{y}, P a_{z}$ demixed from $W a_{n}$ (Molecule names represent first two letters of their names). To further complicate things, an amalgam of surfactants can be added to the system to get the mixture close to a tensionless, balanced state by optimizing both $\omega$ and $\int z p(z)$.

It is easy to imagine the complexities in modeling such systems. Not only does the parameter space (interaction between different species) increase but so does the complications associated with modeling. While analyzing the simple $\mathrm{L} / \mathrm{L}$ interfaces we stressed the importance of remaining in a Grand canonical ensemble $\left(\mu_{A}, \mu_{B}\right)$ is fixed in all the geometries. This was achieved by using a Lagrange parameter $\beta$. By increasing the number of species, the $\beta$ parameter has to be provided more intelligently while ensuring the ratio between different species is maintained as that of the planar interface.

\section{Standing waves to model fluctuations}

An external potential field can be imposed on a standard SF-SCF system. There are numerous ways such an external field can be applied. Fraaije [205] added an external potential field to a homogeneous mixture to drive phase separation and studied the structures that evolved. Such simulations were called Mesodyn and use a random uncorrelated noise at the lattice sites as an external field. Interestingly, they showed that such an external field does not necessarily have to be a potential field. Random noise added to the density field will also result in a similar picture. However, in our work [206], we found that such a random external field was incapable of generating a coordinated movement at the interfaces. Instead of providing an external potential field made up of random noise, a coordinated wave-like field can be imposed.

Such an external field will be capable to imitate wave-like fluctuations and is thus called the Fluctuation enhanced self-consistent field theory (FL-SCF). A range of wavenumbers that fits within the entire box perfectly are chosen and a constant amplitude sinusoidal waves are created and added to the potential. The root-mean-square of the amplitude is normalized to $10^{-4}$ [207-209]. The choice 
of $10^{-4}$ was made based on works of Onuki on sound waves near critical points. An amplitude higher than the chosen value will lead to a non-linear response and destroy the expected wave-like fluctuation behavior of interfaces.

As a first step towards such an approach we tested two systems: 1) where the external wave-like potential is added only on the interfacial plane 2) the external potential is added to the entire system.

\section{FL-SCF: Interfacial plane}

In Chapter 5, we moved a few pinned points on the interface to recover the spectrum of interfacial fluctuations. Discreteness was portrayed as a huge hindrance. It prevented creating waves in a continuous space as the pins are essentially forced to remain in an integer lattice site. Such a disadvantage is overcome by applying an external field. As the potentials can be provided in a continuous space the discreteness issue in MC-SCF is partially addressed in FL-SCF. As expected, we observed a fluctuating interface similar to that observed in an MC-SCF (Not shown).

To refine the discreteness further, one has to consider a system where the segment size is larger than the lattice size. Such a quasi lattice-free theory is being recently published by our group [210]. It was shown that for systems when the segment size is equal to that of the lattice size, a hexagonal lattice proved to be the right choice to recover proper coefficients in Edwards equation. By ignoring the details of such a theory, in principle, it becomes possible to reach a continuous limit upon refining the lattice. In a continuous limit, it then becomes possible to establish waves of any height in the system for a given wavelength. This becomes important when we attempt to model larger wavelengths that have an extremely small amplitude. A discrete lattice will either kill this wave by forcing it to remain in the interfacial plane or shoots up the amplitude by moving the interfacial site a whole layer up.

Finally, FL-SCF on a plane still does not account for the bulk fluctuations. By providing an external field on the plane essentially ignores the bulk. However, an advantage is that the bulk concentrations do not change. This will eventually help perform the simulations withing a Grand canonical ensemble. We decided to impose the potential field for the whole system rather than focussing on a single plane to overcome the exclusion of bulk fluctuations. The preliminary results will be discussed in the next section.

\section{FL-SCF: Whole system}

In Fig. 5.1, we present the density distribution of the system before and after applying an external potential field. The model system discussed in Chapter 2 is used. Two liquids $A_{4}, B_{4}$ at an interaction parameter $\chi=0.51$ is studied. The critical point of the system occurs at $\chi=0.5$ where the system becomes a homogeneous mixture. Simulation is performed in a $32 \times 32 \times 32$-size box. Before 

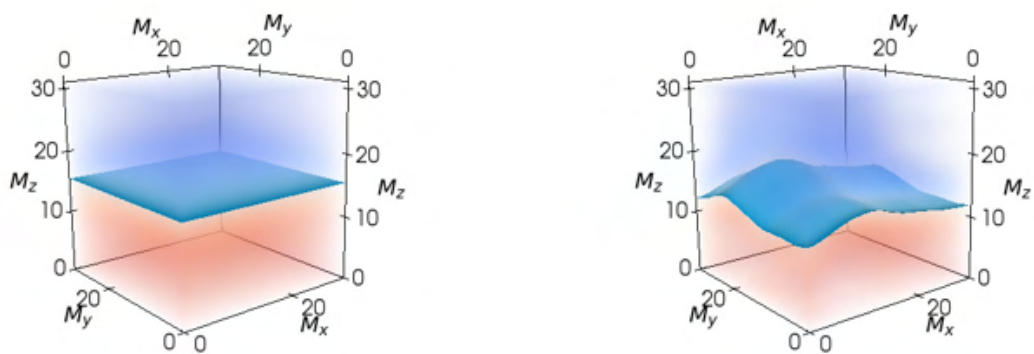

Figure 6.1: Fluctuation enhanced SCF. Over a base equilibrium solution of meanfield model, external potential are applied. These external potentials are sum of several wave-like fluctuations with a constant amplitude of $10^{-4}$. By tuning this amplitude, to represent equipartition theorem will be a way forward to model fluctuations in mean-field theories.

applying an external field, a flat interface separates the liquids. Upon adding an external field, the interface is pushed upon by the standing waves in the system in all directions, the resulting system features a fluctuating interface.

Much remains unknown of FL-SCF. How should the amplitude of the standing waves be modified to imitate thermal fluctuations? Do the fundamental parameters still control the fluctuations that are forced by the external field? The second question can be answered by comparing the change in the grand potential of the system before and after applying the external potential to the individual integrals of the Helfrich equation. For the calculation presented it was observed that the $\Delta \Omega \approx \gamma \int d A+1 / 2 \kappa \int J^{2} d A+\bar{\kappa} \int K d A$. Individual integrals were evaluated as discussed in Chapter 5 .

Unlike the MC-SCF, however, the increase in the grand potential is not selected using a Metropolis algorithm. As a step forward, a hybrid Monte Carlo simulation that uses the external fields to make a move, where the amplitude of the external field is selected randomly $\left(0<A<10^{-4}\right)$ can recover the spectrum behavior. However, an easier way forward would be to tune the individual amplitude of each wave that is imposed in the external field to recover the spectrum. Further, the chemical potentials are not strictly fixed in these simulations compared to the FL-SCF applied to the interfacial plane. However, if several such calculations are sampled, the expected value of the chemical potential $\langle\mu\rangle$ will converge to ground state $\mu_{p}$. 


\subsection{Conclusion}

To conclude, we have strongly laid the fundamentals to model microemulsions in this thesis work. In this chapter, we discussed how the ideal models can be used as a skeleton to build up more realistic complicated models. We believe that the inclusion of asymmetry and fluctuation enhancement using external fields will allow us to realistically model crude oil recovery, drug adsorption, etc. using SF-SCF in the future. 


\section{Bibliography}

${ }^{1}$ Schwarze, M., Pogrzeba, T., Volovych, I., and Schomäcker, R., "Microemulsion systems for catalytic reactions and processes", Catalysis Science \& Technology vol. 5, no. 1, 24-33 (2015).

${ }^{2}$ Kahlweit, M., Strey, R., and Firman, P., "Search for tricritical points in ternary systems: water-oil-nonionic amphiphile", The Journal of Physical Chemistry vol. 90, no. 4, 671-677 (1986).

${ }^{3}$ Porter, C. J., Trevaskis, N. L., and Charman, W. N., "Lipids and lipid-based formulations: optimizing the oral delivery of lipophilic drugs", Nature reviews Drug discovery vol. 6, no. 3, 231-248 (2007).

${ }^{4}$ Danielsson, I. and Lindman, B., "The definition of microemulsion", Colloids and Surfaces vol. 3, no. 4, 391-392 (1981).

${ }^{5}$ Franklin, B. and Brownrigg, W., "Xliv. of the stilling of waves by means of oil. extracted from sundry letters between benjamin franklin, ll. dfrs william brownrigg, mdfrs and the reverend mr. farish", Philosophical Transactions of the Royal Society of London, no. 64, 445-460 (1774).

${ }^{6}$ Flanagan, J. and Singh, H., "Microemulsions: a potential delivery system for bioactives in food", Critical reviews in food science and nutrition vol. 46, no. 3, 221-237 (2006).

${ }^{7}$ Spernath, A., Yaghmur, A., Aserin, A., Hoffman, R. E., and Garti, N., "Self-diffusion nuclear magnetic resonance, microstructure transitions, and solubilization capacity of phytosterols and cholesterol in winsor iv food-grade microemulsions", Journal of agricultural and food chemistry vol. 51, no. 8, 2359 2364 (2003). 
${ }^{8}$ Spernath, A., Yaghmur, A., Aserin, A., Hoffman, R. E., and Garti, N., "Foodgrade microemulsions based on nonionic emulsifiers: media to enhance lycopene solubilization", Journal of Agricultural and Food Chemistry vol. 50, no. 23, 6917-6922 (2002).

${ }^{9}$ Paul, B. K. and Moulik, S. P., "Uses and applications of microemulsions", Current science, 990-1001 (2001).

${ }^{10}$ Qi, J., Zhuang, J., Wu, W., Lu, Y., Song, Y., Zhang, Z., Jia, J., and Ping, Q., "Enhanced effect and mechanism of water-in-oil microemulsion as an oral delivery system of hydroxysafflor yellow a", International journal of nanomedicine vol. 6 , 985 (2011).

${ }^{11} \mathrm{McClements,} \mathrm{D.} \mathrm{J.} \mathrm{and} \mathrm{Li,} \mathrm{Y.,} \mathrm{"Structured} \mathrm{emulsion-based} \mathrm{delivery} \mathrm{systems:}$ controlling the digestion and release of lipophilic food components", Advances in colloid and interface science vol. 159, no. 2, 213-228 (2010).

${ }^{12}$ Singh, H., Ye, A., and Horne, D., "Structuring food emulsions in the gastrointestinal tract to modify lipid digestion", Progress in lipid research vol. 48, no. 2, 92-100 (2009).

${ }^{13}$ Walsh, F. R. and Zoback, M. D., "Oklahoma's recent earthquakes and saltwater disposal", Science advances vol. 1, no. 5, e1500195 (2015).

${ }^{14}$ Ong, B., "The potential impacts of hydraulic fracturing on agriculture", European Journal of Sustainable Development vol. 3, no. 3, 63-63 (2014).

${ }^{15}$ Warner, N. R., Jackson, R. B., Darrah, T. H., Osborn, S. G., Down, A., Zhao, K., White, A., and Vengosh, A., "Geochemical evidence for possible natural migration of marcellus formation brine to shallow aquifers in pennsylvania", Proceedings of the National Academy of Sciences vol. 109, no. 30, 11961-11966 (2012).

${ }^{16}$ Myers, T., "Potential contaminant pathways from hydraulically fractured shale to aquifers", Groundwater vol. 50, no. 6, 872-882 (2012).

${ }^{17}$ Jackson, R. B., Vengosh, A., Darrah, T. H., Warner, N. R., Down, A., Poreda, R. J., Osborn, S. G., Zhao, K., and Karr, J. D., "Increased stray gas abundance in a subset of drinking water wells near marcellus shale gas extraction", Proceedings of the National Academy of Sciences vol. 110, no. 28, 11250-11255 (2013).

${ }^{18}$ Osborn, S. G., Vengosh, A., Warner, N. R., and Jackson, R. B., "Methane contamination of drinking water accompanying gas-well drilling and hydraulic fracturing", proceedings of the National Academy of Sciences vol. 108, no. 20, $8172-8176$ (2011). 
${ }^{19}$ Ye, M., Han, Y., Tang, J., Piao, Y., Liu, X., Zhou, Z., Gao, J., Rao, J., and Shen, Y., "A tumor-specific cascade amplification drug release nanoparticle for overcoming multidrug resistance in cancers", Advanced Materials vol. 29, no. 38, 1702342 (2017).

${ }^{20} \mathrm{Li}, \mathrm{W}$., Wang, H., and Yang, Y., "Protein-modulated release behavior of a hydrophilic/lipophilic dual-drug from supramolecularly gelled microemulsions", RSC Advances vol. 4, no. 5, 2109-2114 (2014).

${ }^{21}$ Ghayempour, S. and Montazer, M., "Tragacanth nanocapsules containing chamomile extract prepared through sono-assisted w/o/w microemulsion and uv cured on cotton fabric", Carbohydrate polymers vol. 170, 234-240 (2017).

${ }^{22}$ Haase, M. F., Stebe, K. J., and Lee, D., "Continuous fabrication of hierarchical and asymmetric bijel microparticles, fibers, and membranes by solvent transferinduced phase separation (strips)", Advanced Materials vol. 27, no. 44, 7065-7071 (2015).

${ }^{23}$ Herzig, E. M., White, K., Schofield, A. B., Poon, W. C., and Clegg, P. S., "Bicontinuous emulsions stabilized solely by colloidal particles", Nature materials vol. 6, no. 12, 966-971 (2007).

${ }^{24}$ Stratford, K., Adhikari, R., Pagonabarraga, I., Desplat, J.-C., and Cates, M. E., "Colloidal jamming at interfaces: a route to fluid-bicontinuous gels", Science vol. 309, no. 5744, 2198-2201 (2005).

${ }^{25}$ Stouch, T. R., "Lipid membrane structure and dynamics studied by all-atom molecular dynamics simulations of hydrated phospholipid bilayers", Molecular Simulation vol. 10, no. 2-6, 335-362 (1993).

${ }^{26}$ Hoston, W. and Berker, A. N., "Multicritical phase diagrams of the blume-emerygriffiths model with repulsive biquadratic coupling", Physical review letters vol. 67, no. 8, 1027 (1991).

${ }^{27}$ Wang, Y.-L. and Wentworth, C., "Phase diagrams of three-dimensional blumeemery-griffiths model", Journal of Applied Physics vol. 61, no. 8, 4411-4412 (1987).

${ }^{28}$ Selke, W. and Yeomans, J., "Interface properties of the two-dimensional blumeemery-griffiths model", Journal of Physics A: Mathematical and General vol. 16, no. 12, 2789 (1983).

${ }^{29}$ Lerczak, J., Schick, M., and Gompper, G., "Variation with amphiphilic strength of the properties of ternary mixtures", Physical Review A vol. 46, no. 2, 985 (1992).

${ }^{30}$ Gompper, G. and Schick, M., "Microemulsion structure from a three-component lattice model", Physical review letters vol. 62, no. 14, 1647 (1989). 
${ }^{31}$ Gompper, G. and Schick, M., "Correlation between structural and interfacial properties of amphiphilic systems", Physical review letters vol. 65, no. 9, 1116 (1990).

${ }^{32}$ Nightingale, M. P., "Scaling theory and finite systems", Physica A: Statistical Mechanics and its Applications vol. 83, no. 3, 561-572 (1976).

${ }^{33}$ Laradji, M. and Mouritsen, O. G., "Elastic properties of surfactant monolayers at liquid-liquid interfaces: a molecular dynamics study", The Journal of Chemical Physics vol. 112, no. 19, 8621-8630 (2000).

${ }^{34}$ Blokhuis, E. M. and Bedeaux, D., "Derivation of microscopic expressions for the rigidity constants of a simple liquid-vapor interface", Physica A: Statistical Mechanics and its Applications vol. 184, no. 1-2, 42-70 (1992).

${ }^{35}$ Matsen, M. W., "Elastic properties of a diblock copolymer monolayer and their relevance to bicontinuous microemulsion", The Journal of Chemical Physics vol. 110, no. 9, 4658-4667 (1999).

${ }^{36}$ Leermakers, F. A. M., "Direct evaluation of the saddle splay modulus of a liquidliquid interface using the classical mean field lattice model", The Journal of Chemical Physics vol. 138, no. 12, 124103 (2013).

${ }^{37}$ Oversteegen, S. M. and Blokhuis, E. M., "Rigidity constants from mean-field models", The Journal of Chemical Physics vol. 112, no. 6, 2980-2986 (2000).

${ }^{38}$ Blokhuis, E., Groenewold, J., and Bedeaux, D., "Fluctuation route to the bending rigidity", Molecular Physics vol. 96, no. 3, 397-406 (1999).

${ }^{39}$ Van Giessen, A. E. and Blokhuis, E. M., "Determination of curvature corrections to the surface tension of a liquid-vapor interface through molecular dynamics simulations", The Journal of chemical physics vol. 116, no. 1, 302-310 (2002).

${ }^{40}$ Müller, M. and Gompper, G., "Elastic properties of polymer interfaces: aggregation of pure diblock, mixed diblock, and triblock copolymers", Physical Review E vol. 66, no. 4, 041805 (2002).

${ }^{41}$ Müller, M. and Schmid, F., "Incorporating fluctuations and dynamics in selfconsistent field theories for polymer blends", in Advanced computer simulation approaches for soft matter sciences ii (Springer, 2005), pp. 1-58.

${ }^{42}$ Laradji, M. and Desai, R. C., "Elastic properties of homopolymer-homopolymer interfaces containing diblock copolymers", The Journal of chemical physics vol. 108, no. 11, 4662-4674 (1998).

${ }^{43}$ Van der Waals, J. D., "Over de continuiteit van den gas- en vloeistof toestand", PhD thesis (Leiden, the Netherlands, 1873).

${ }^{44}$ Van der Waals, J. D., "The thermodynamic theory of capillarity under the hypothesis of a continuous variation of density", Journal of Statistical Physics vol. 20, no. 2, 200-244 (1979). 
${ }^{45}$ Safran, S. A., Statistical thermodynamics of surfaces, interfaces, and membranes, Vol. 90 (Perseus Books, New York, 1994).

${ }^{46}$ Semenov, A. N., "Theory of block copolymer interfaces in the strong segregation limit", Macromolecules vol. 26, no. 24, 6617-6621 (1993).

${ }^{47}$ Helfrich, W., "Elastic properties of lipid bilayers: theory and possible experiments", Z. Naturforsch. C vol. 28, no. 11-12, 693-703 (1973).

${ }^{48}$ Rowlinson, J. S. and Widom, B., Molecular theory of capillarity (Courier Corporation, 2013).

${ }^{49}$ Mitrinović, D. M., Tikhonov, A. M., Li, M., Huang, Z., and Schlossman, M. L., "Noncapillary-wave structure at the water-alkane interface", Physical review letters vol. 85, no. 3, 582 (2000).

${ }^{50}$ Wu, X. Z., Sirota, E. B., Sinha, S. K., Ocko, B. M., and Deutsch, M., "Surface crystallization of liquid normal-alkanes", Physical review letters vol. 70, no. 7, 958 (1993).

${ }^{51}$ Ocko, B. M., Wu, X. Z., Sirota, E. B., Sinha, S. K., and Deutsch, M., "X-ray reflectivity study of thermal capillary waves on liquid surfaces", Physical review letters vol. 72, no. 2, 242 (1994).

${ }^{52}$ Merkl, C., Pfohl, T., and Riegler, H., "Influence of the molecular ordering on the wetting of sio 2/air interfaces by alkanes", Physical review letters vol. 79, no. 23, 4625 (1997).

${ }^{53}$ Scheutjens, J. M. H. M. and Fleer, G. J., "Statistical theory of the adsorption of interacting chain molecules. 1. partition function, segment density distribution, and adsorption isotherms", J. phys. Chem vol. 83, no. 12, 1619-1635 (1979).

${ }^{54}$ Scheutjens, J. M. H. M. and Fleer, G. J., "Statistical theory of the adsorption of interacting chain molecules. 2. train, loop, and tail size distribution", J. Phys. Chem. vol. 84, no. 2, 178-190 (1980).

${ }^{55}$ Leermakers, F. A. M., "Bending rigidities of surfactant bilayers using selfconsistent field theory", J. Chem. Phys. vol. 138, no. 15, 04B610 (2013).

${ }^{56}$ Kik, R. A., Leermakers, F. A. M., and Kleijn, J. M., "Molecular modeling of proteinlike inclusions in lipid bilayers: lipid-mediated interactions", Phys. Rev. E vol. 81, no. 2, 021915 (2010).

${ }^{57}$ Fleer, G., Stuart, M. A. C., Scheutjens, J. M. H. M., Cosgrove, T., and Vincent, B., Polymers at interfaces (Springer Science \& Business Media, 1993).

${ }^{58}$ Evers, O. A., Scheutjens, J. M. H. M., and Fleer, G. J., "Statistical thermodynamics of block copolymer adsorption. 1 . formulation of the model and results for the adsorbed layer structure", Macromolecules vol. 23, no. 25, 5221-5233 (1990). 
${ }^{59}$ See Supplemental Material http://link.aps.org/ which includes Refs. [4, 13, 20 \& $28]$.

${ }^{60}$ Helfand, E. and Tagami, Y., "Theory of the interface between immiscible polymers", Journal of Polymer Science Part B: Polymer Letters vol. 9, no. 10, 741-746 (1971).

${ }^{61}$ Helfand, E., "Theory of inhomogeneous polymers: fundamentals of the gaussian random-walk model", The Journal of Chemical Physics vol. 62, no. 3, 999-1005 (1975).

${ }^{62}$ Semenov, A. N., "Scattering of statistical structure of polymer/polymer interfaces", Macromolecules vol. 27, no. 10, 2732-2735 (1994).

${ }^{63}$ Müller, M., Binder, K., and Oed, W., "Structural and thermodynamic properties of interfaces between coexisting phases in polymer blends: a monte carlo simulation", Journal of the Chemical Society, Faraday Transactions vol. 91, no. 16, 2369-2379 (1995).

${ }^{64}$ Varadharajan, R. and Leermakers, F. A. M., "Sign switch of gaussian bending modulus for microemulsions: a self-consistent field analysis exploring scale invariant curvature energies", Physical Review Letters vol. 120, no. 2, 028003 (2018).

${ }^{65}$ Mocan, M., Kamperman, M., and Leermakers, F. A. M., "Microphase segregation of diblock copolymers studied by the self-consistent field theory of scheutjens and fleer", Polymers vol. 10, no. 1, 78 (2018).

${ }^{66}$ Blokhuis, E. M., "On the spectrum of fluctuations of a liquid surface: from the molecular scale to the macroscopic scale", The Journal of Chemical Physics vol. 130, no. 1, 014706 (2009).

${ }^{67}$ Leermakers, F. A. M., Skvortsov, A. M., and Klushin, L. I., "Negative compressibility for a polymer chain squeezed between two pistons going through the escape transition", Journal of Statistical Mechanics: Theory and Experiment vol. 2004, no. 10, P10001 (2004).

${ }^{68}$ Blokhuis, E. M., Kuipers, J., and Vink, R. L. C., "Description of the fluctuating colloid-polymer interface", Physical review letters vol. 101, no. 8, 086101 (2008).

${ }^{69} \mathrm{Xu}$, L., Zhang, W. W., and Nagel, S. R., "Drop splashing on a dry smooth surface", Physical review letters vol. 94, no. 18, 184505 (2005).

${ }^{70}$ Yarin, A. L., "Drop impact dynamics: splashing, spreading, receding, bouncing...", Annu. Rev. Fluid Mech. vol. 38, 159-192 (2006).

${ }^{71}$ Tekin, E., Smith, P. J., and Schubert, U. S., "Inkjet printing as a deposition and patterning tool for polymers and inorganic particles", Soft Matter vol. 4, no. 4 , 703-713 (2008). 
${ }^{72}$ Duez, C., Ybert, C., Clanet, C., and Bocquet, L., "Making a splash with water repellency", Nature physics vol. 3, no. 3, 180 (2007).

${ }^{73}$ Gennes, P. G. de and Taupin, C., "Microemulsions and the flexibility of oil/water interfaces", J. Phys. Chem. vol. 86, no. 13, 2294-2304 (1982).

${ }^{74}$ Guha, I. F., Anand, S., and Varanasi, K. K., "Creating nanoscale emulsions using condensation", Nature Communications vol. 8, no. 1, 1371 (2017).

${ }^{75}$ Labrador, A., Seddon, A. M., Squires, A. M., Dicko, C., Pfrang, C., CabreraMartinez, E. R., Rastogi, K., Cowieson, N., and Plivelic, T. S., "Complex three-dimensional self-assembly in proxies for atmospheric aerosols", Nature Communications vol. 8, no. 1, 1724 (2017).

${ }^{76}$ Tanford, C., The hydrophobic effect: formation of micelles and biological membranes (J. Wiley., 1980).

${ }^{77}$ Schacht, S., Huo, Q., Voigt-Martin, I. G., Stucky, G. D., and Schuth, F., "Oilwater interface templating of mesoporous macroscale structures", Science vol. 273, no. 5276, 768 (1996).

${ }^{78}$ Gompper, G., Schick, M., and Milner, S., Self-assembling amphiphilic systems, 1995.

${ }^{79}$ Safran, S. A., Roux, D., Cates, M. E., and Andelman, D., "Origin of middle-phase microemulsions", Phys. Rev. Lett. vol. 57, no. 4, 491 (1986).

${ }^{80}$ Scriven, L., "Equilibrium bicontinuous structure", Nature (London) vol. 263, no. $5573,123-125$ (1976).

${ }^{81}$ Cates, M., Roux, D., Andelman, D., Milner, S. T., and Safran, S. A., "Random surface model for the 13-phase of dilute surfactant solutions", EPL (Europhys. Lett.) vol. 5, no. 8, 733 (1988).

${ }^{82}$ Hellweg, T. and Langevin, D., "Bending elasticity of the surfactant monolayer in droplet microemulsions: determination by a combination of dynamic light scattering and neutron spin-echo spectroscopy", Phys. Rev. E vol. 57, 6825-6834 (1998).

${ }^{83}$ Kurtisovski, E., Taulier, N., Ober, R., Waks, M., and Urbach, W., "Molecular origin of model membrane bending rigidity", Phys. Rev. Lett. vol. 98, no. 25, 258103 (2007).

${ }^{84}$ Szleifer, I., Kramer, D., Ben-Shaul, A., Roux, D., and Gelbart, W. M., "Curvature elasticity of pure and mixed surfactant films", Phys. Rev. Lett. vol. 60, no. 19, 1966 (1988).

${ }^{85}$ Würger, A., "Bending elasticity of surfactant films: the role of the hydrophobic tails", Phys. Rev. Lett. vol. 85, no. 2, 337 (2000). 
${ }^{86}$ Gradzielski, M., Langevin, D., and Farago, B., "Experimental investigation of the structure of nonionic microemulsions and their relation to the bending elasticity of the amphiphilic film", Phys. Rev. E vol. 53, no. 4, 3900 (1996).

${ }^{87}$ Gradzielski, M., Langevin, D., Sottmann, T., and Strey, R., "Droplet microemulsions at the emulsification boundary: the influence of the surfactant structure on the elastic constants of the amphiphillic film", J. Chem. Phys. vol. 106, no. 19, 8232-8238 (1997).

${ }^{88}$ Safinya, C. R., Sirota, E. B., Roux, D., and Smith, G. S., "Universality in interacting membranes: the effect of cosurfactants on the interfacial rigidity", Physical review letters vol. 62, no. 10, 1134 (1989).

${ }^{89}$ Rekvig, L., Hafskjold, B., and Smit, B., "Chain length dependencies of the bending modulus of surfactant monolayers", Phys. Rev. Lett. vol. 92, 116101 (2004).

${ }^{90}$ Thakkar, F. M., Maiti, P. K., Kumaran, V., and Ayappa, K. G., "Verifying scalings for bending rigidity of bilayer membranes using mesoscale models", Soft Matter vol. 7, 3963-3966 (2011).

${ }^{91}$ Cosgrove, T., Heath, T., Van Lent, B., Leermakers, F., and Scheutjens, J., "Configuration of terminally attached chains at the solid/solvent interface: selfconsistent field theory and a monte carlo model", Macromolecules vol. 20, no. 7 , 1692-1696 (1987).

${ }^{92}$ Hurter, P. N., Scheutjens, J. M. H. M., and Hatton, T. A., "Molecular modeling of micelle formation and solubilization in block copolymer micelles. 1. a selfconsistent mean-field lattice theory", Macromolecules vol. 26, no. 21, 5592-5601 (1993).

${ }^{93}$ Wijmans, C. M., Scheutjens, J. M. H. M., and Zhulina, E. B., "Self-consistent field theories for polymer brushes: lattice calculations and an asymptotic analytical description", Macromolecules vol. 25, no. 10, 2657-2665 (1992).

${ }^{94}$ Oversteegen, S. M., Barneveld, P. A., Male, J. V., Leermakers, F. A. M., et al., "Thermodynamic derivation of mechanical expressions for interfacial parameters", Physical Chemistry Chemical Physics vol. 1, no. 21, 4987-4994 (1999).

${ }^{95}$ Chern, S. S., "A simple intrinsic proof of the gauss-bonnet formula for closed riemannian manifolds", Annals of mathematics, 747-752 (1944).

${ }^{96}$ Fenchel, W., "On total curvatures of riemannian manifolds: i", Journal of the London Mathematical Society vol. 1, no. 1, 15-22 (1940).

${ }^{97}$ Willmore, T. J., "Note on embedded surfaces", An. Sti. Univ. "Al. I. Cuza" Iasi Sect. I a Mat.(NS) B vol. 11, 493-496 (1965).

${ }^{98}$ Marques, F. C. and Neves, A., "Min-max theory and the willmore conjecture", Annals of Mathematics vol. 179, 683-782 (2014). 
${ }^{99}$ Smit, B., Hilbers, P. A. J., Esselink, K., Rupert, L. A. M., Van Os, N. M., and Schlijper, A. G., "Computer simulations of a water/oil interface in the presence of micelles", Nature (London) vol. 348, no. 6302, 624-625 (1990).

${ }^{100}$ Yamamoto, J. and Tanaka, H., "Transparent nematic phase in a liquid-crystalbased microemulsion", Nature vol. 409, no. 6818, 321 (2001).

${ }^{101}$ Thiam, A. R., Farese Jr, R. V., and Walther, T. C., "The biophysics and cell biology of lipid droplets", Nature reviews Molecular cell biology vol. 14, no. 12, 775 (2013).

${ }^{102}$ Chang, K., Macosko, C. W., and Morse, D. C., "Ultralow interfacial tensions of polymer/polymer interfaces with diblock copolymer surfactants", Macromolecules vol. 40, no. 10, 3819-3830 (2007).

${ }^{103}$ Clausse, M., Peyrelasse, J., Heil, J., Boned, C., and Lagourette, B., "Bicontinuous structure zones in microemulsions", Nature vol. 293, no. 5834, 636-638 (1981).

${ }^{104}$ Walsh, D., Hopwood, J. D., and Mann, S., "Crystal tectonics: construction of reticulated calcium phosphate frameworks in bicontinuous reverse microemulsions", Science vol. 264, no. 5165, 1576-1578 (1994).

${ }^{105}$ Morales, D., Gutiérrez, J. M., Garcia-Celma, M. J., and Solans, Y. C., "A study of the relation between bicontinuous microemulsions and oil/water nano-emulsion formation", Langmuir vol. 19, no. 18, 7196-7200 (2003).

${ }^{106}$ Jones, B. H. and Lodge, T. P., "Nanocasting nanoporous inorganic and organic materials from polymeric bicontinuous microemulsion templates", Polymer journal vol. 44, no. 2, 131 (2012).

${ }^{107}$ Neubauer, R., Höhn, S., Dulle, M., Lapp, A., Schulreich, C., and Hellweg, T., "Protein diffusion in a bicontinuous microemulsion: inducing sub-diffusion by tuning the water domain size", Soft matter vol. 13, no. 10, 1998-2003 (2017).

${ }^{108}$ Flory, P. J., Principles of polymer chemistry (Cornell University Press, 1953).

${ }^{109}$ Düchs, D., Ganesan, V., Fredrickson, G. H., and Schmid, F., "Fluctuation effects in ternary $\mathrm{ab}+\mathrm{a}+\mathrm{b}$ polymeric emulsions", Macromolecules vol. 36, no. 24, 9237-9248 (2003).

${ }^{110}$ Naughton, J. R. and Matsen, M. W., "Nonperiodic lamellar phase in ternary diblock copolymer/homopolymer blends", Macromolecules vol. 35, no. 24, 89268928 (2002).

${ }^{111}$ Naughton, J. R. and Matsen, M. W., "Limitations of the dilution approximation for concentrated block copolymer/solvent mixtures", Macromolecules vol. 35, no. 14, 5688-5696 (2002).

${ }^{112}$ Leibler, L., "Theory of phase equilibria in mixtures of copolymers and homopolymers, 1. phase diagram", Die Makromolekulare Chemie, Rapid Communications vol. 2, no. 6-7, 393-400 (1981). 
${ }^{113}$ Broseta, D. and Fredrickson, G. H., "Phase equilibria in copolymer/homopolymer ternary blends: molecular weight effects", The Journal of Chemical Physics vol. 93, no. 4, 2927-2938 (1990).

${ }^{114}$ Van Konynenburg, P. and Scott, R., "Critical lines and phase equilibria in binary van der waals mixtures", Philosophical Transactions of the Royal Society of London. Series A, Mathematical and Physical Sciences vol. 298, no. 1442, 495540 (1980).

${ }^{115}$ Leibler, L., "Theory of microphase separation in block copolymers", Macromolecules vol. 13, no. 6, 1602-1617 (1980).

${ }^{116}$ Holyst, R. and Schick, M., "Copolymers as amphiphiles in ternary mixtures: an analysis employing disorder, equimaxima, and lifshitz lines", The Journal of chemical physics vol. 96, no. 10, 7728-7737 (1992).

${ }^{117}$ Morkved, T. L., Stepanek, P., Krishnan, K., Bates, F. S., and Lodge, T. P., "Static and dynamic scattering from ternary polymer blends: bicontinuous microemulsions, lifshitz lines, and amphiphilicity", The Journal of Chemical Physics vol. 114, no. 16, 7247-7259 (2001).

${ }^{118}$ Kielhorn, L. and Muthukumar, M., "Spinodal decomposition of symmetric diblock copolymer/homopolymer blends at the lifshitz point", The Journal of chemical physics vol. 110, no. 8, 4079-4089 (1999).

${ }^{119}$ Kielhorn, L. and Muthukumar, M., "Fluctuation theory of diblock copolymer/homopolymer blends and its effects on the lifshitz point", The Journal of chemical physics vol. 107, no. 14, 5588-5608 (1997).

${ }^{120}$ Chang, K. and Morse, D. C., "Diblock copolymer surfactants in immiscible homopolymer blends: interfacial bending elasticity", Macromolecules vol. 39, no. $21,7397-7406$ (2006).

${ }^{121}$ Leermakers, F. A. M., Scheutjens, J. M. H. M., and Lyklema, J., "On the statistical thermodynamics of membrane formation", Biophysical chemistry vol. 18, no. 4, 353-360 (1983).

${ }^{122}$ Leermakers, F. A. M. and Scheutjens, J. M. H. M., "Statistical thermodynamics of association colloids. 2. lipid vesicles", The Journal of Physical Chemistry vol. 93, no. 21, 7417-7426 (1989).

${ }^{123}$ Leermakers, F. A. M. and Scheutjens, J. M. H. M., "Statistical thermodynamics of association colloids. i. lipid bilayer membranes", The Journal of chemical physics vol. 89, no. 5, 3264-3274 (1988).

${ }^{124}$ Leermakers, F. A. M., "Statistical thermodynamics of association colloids: the equilibrium structure of micelles, vesicles, and bilayer membranes", $\mathrm{PhD}$ thesis (Landbouwuniversiteit Wageningen, 1988). 
${ }^{125}$ Bates, F. S., Maurer, W. W., Lipic, P. M., Hillmyer, M. A., Almdal, K., Mortensen, K., Fredrickson, G. H., and Lodge, T. P., "Polymeric bicontinuous microemulsions", Physical review letters vol. 79, no. 5, 849 (1997).

${ }^{126}$ Hillmyer, M. A., Maurer, W. W., Lodge, T. P., Bates, F. S., and Almdal, K., "Model bicontinuous microemulsions in ternary homopolymer/block copolymer blends", The Journal of Physical Chemistry B vol. 103, no. 23, 4814-4824 (1999).

${ }^{127}$ Dimitrov, D. I., Klushin, L. I., Skvortsov, A., Milchev, A., and Binder, K., "The escape transition of a polymer: a unique case of non-equivalence between statistical ensembles", The European Physical Journal E vol. 29, no. 1, 9-25 (2009).

${ }^{128}$ Skvortsov, A. M., Klushin, L. I., and Leermakers, F. A. M., "Exactly solved polymer models with conformational escape transitions of a coil-to-flower type", EPL (Europhysics Letters) vol. 58, no. 2, 292 (2002).

${ }^{129}$ Skvortsov, A. M., Gorbunov, A. A., Leermakers, F. A. M., and Fleer, G. J., "Long minority chains in a polymer brush: a first-order adsorption transition", Macromolecules vol. 32, no. 6, 2004-2015 (1999).

${ }^{130}$ Evans, D. F. and Wennerström, H., The colloidal domain: where physics, chemistry, biology, and technology meet (Wiley-VCH New York, 1999).

${ }^{131}$ Ehrenfest, P., Phasenumwandlungen im ueblichen und erweiterten sinn, classifiziert nach den entsprechenden singularitaeten des thermodynamischen potentiales (NV Noord-Hollandsche Uitgevers Maatschappij, 1933).

${ }^{132}$ Landau, L. D., Lifshitz, E. M., and Pitaevskii, L. P., Statistical physics, part $i$, 1980 .

${ }^{133}$ Lyklema, J., Fundamentals of interface and colloid science (Academic press, London, 1991).

${ }^{134}$ Varadharajan, R. and Leermakers, F. A. M., "Elastic properties of symmetric liquid-liquid interfaces", arXiv preprint arXiv:1908.02522 (2019).

${ }^{135}$ Safran, S. A., "Curvature elasticity of thin films", Advances in Physics vol. 48, no. 4, 395-448 (1999).

${ }^{136}$ Lekkerkerker, H. N. W., "Contribution of the electric double layer to the curvature elasticity of charged amphiphilic monolayers", Physica A: Statistical Mechanics and its Applications vol. 159, no. 3, 319-328 (1989).

${ }^{137}$ Birshtein, T. M., Iakovlev, P. A., Amoskov, V. M., Leermakers, F. A. M., Zhulina, E. B., and Borisov, O. V., "On the curvature energy of a thin membrane decorated by polymer brushes", Macromolecules vol. 41, no. 2, 478-488 (2008).

${ }^{138}$ Corswant, C. von, Engström, S., and Söderman, O., "Microemulsions based on soybean phosphatidylcholine and triglycerides. phase behavior and microstructure", Langmuir vol. 13, no. 19, 5061-5070 (1997). 
${ }^{139}$ Seifert, U., "Configurations of fluid membranes and vesicles", Advances in physics vol. 46, no. 1, 13-137 (1997).

${ }^{140}$ Peliti, L. and Leibler, S., "Effects of thermal fluctuations on systems with small surface tension", Physical review letters vol. 54, no. 15, 1690 (1985).

${ }^{141}$ Lipowsky, R., "The conformation of membranes", Nature vol. 349, no. 6309, 475 (1991).

${ }^{142}$ Lipowsky, R. and Leibler, S., "Unbinding transitions of interacting membranes", Physical Review Letters vol. 56, no. 23, 2541 (1986).

${ }^{143}$ Roux, D. and Safinya, C. R., "A synchrotron x-ray study of competing undulation and electrostatic interlayer interactions in fluid multimembrane lyotropic phases", Journal de Physique vol. 49, no. 2, 307-318 (1988).

${ }^{144}$ Kahlweit, M. and Strey, R., "Phase behavior of ternary systems of the type h2o oil nonionic amphiphile (microemulsions)", Angewandte Chemie International Edition vol. 24, no. 8, 654-668 (1985).

${ }^{145}$ Kahlweit, M., Strey, R., Firman, P., Haase, D., Jen, J., and Schomäcker, R., "General patterns of the phase behavior of mixtures of water, nonpolar solvents, amphiphiles, and electrolytes. 1", Langmuir vol. 4, no. 3, 499-511 (1988).

${ }^{146}$ Kahlweit, M., Strey, R., Haase, D., and Firman, P., "Properties of the threephase bodies in water-oil-nonionic amphiphile mixtures", Langmuir vol. 4, no. 4, 785-790 (1988).

${ }^{147}$ Banerjee, T., Sarkar, N., Toner, J., and Basu, A., "Rolled up or crumpled: phases of asymmetric tethered membranes", Physical Review Letters vol. 122, no. 21, 218002 (2019).

${ }^{148}$ Yadav, M., Bates, F. S., and Morse, D. C., "Network model of the disordered phase in symmetric diblock copolymer melts", Physical review letters vol. 121, no. 12,127802 (2018).

${ }^{149}$ Beardsley, T. M., Spencer, R. K. W., and Matsen, M. W., "Computationally efficient field-theoretic simulations for block copolymer melts", Macromolecules (2019).

${ }^{150}$ Waals van der, J., Doctoral dissertation, 1973.

${ }^{151}$ Lawrence, M. J. and Rees, G. D., "Microemulsion-based media as novel drug delivery systems", Advanced drug delivery reviews vol. 45, no. 1, 89-121 (2000).

${ }^{152}$ Tenjarla, S., "Microemulsions: an overview and pharmaceutical applications", Critical Reviews ${ }^{\mathrm{TM}}$ in Therapeutic Drug Carrier Systems vol. 16, no. 5 (1999).

${ }^{153}$ Venkataraman, P., Tang, J., Frenkel, E., McPherson, G. L., He, J., Raghavan, S. R., Kolesnichenko, V., Bose, A., and John, V. T., "Attachment of a hydrophobically modified biopolymer at the oil-water interface in the treatment of oil spills", ACS applied materials \& interfaces vol. 5, no. 9, 3572-3580 (2013). 
Bibliography

${ }^{154}$ Nikishov, V. and Nikishov, V., "Spectrum of turbulent fluctuations of the seawater refraction index", International journal of fluid mechanics research vol. 27, no. 1 (2000).

${ }^{155}$ Varadharajan, R. and Leermakers, F. A., "The physics of microemulsions extracted from modeling balanced tensionless surfactant-loaded liquid-liquid interfaces", The Journal of Chemical Physics vol. 152, no. 9, 094902 (2020).

${ }^{156}$ Zhong-Can, O.-Y. and Helfrich, W., "Instability and deformation of a spherical vesicle by pressure", Physical review letters vol. 59, no. 21, 2486 (1987).

${ }^{157}$ Wiese, W., Harbich, W., and Helfrich, W., "Budding of lipid bilayer vesicles and flat membranes", Journal of physics: Condensed matter vol. 4, no. 7, 1647 (1992).

${ }^{158}$ Helfrich, W. and Servuss, R. .-M., "Undulations, steric interaction and cohesion of fluid membranes", Il Nuovo Cimento D vol. 3, no. 1, 137-151 (1984).

${ }^{159}$ Helfrich, W., "Effect of thermal undulations on the rigidity of fluid membranes and interfaces", J. Phys. Paris vol. 46, 1263-1269 (1985).

${ }^{160}$ Helfrich, W., "Measures of integration in calculating the effective rigidity of fluid surfaces", J. Phys. Paris vol. 48, 285-289 (1987).

${ }^{161}$ Gelbart, W. M., Ben-Shaul, A., and Roux, D., Micelles, membranes, microemulsions, and monolayers (Springer Science \& Business Media, 2012).

${ }^{162}$ Bergsma, J., Gucht, J. van der, and Leermakers, F. A., "Coarse-grained dendrimers in a good solvent: comparison of monte carlo simulations, selfconsistent field theory, and a hybrid modeling strategy", Macromolecular Theory and Simulations vol. 28, no. 4, 1800064 (2019).

${ }^{163}$ Bergsma, J., Leermakers, F., and Gucht, J. van der, "Interactions between nodes in a physical gel network of telechelic polymers; self-consistent field calculations beyond the cell model", Physical Chemistry Chemical Physics vol. 17, no. 14, 9001-9014 (2015).

${ }^{164}$ Bergsma, J., Leermakers, F., Kleijn, J., and Van Der Gucht, J., "A hybrid monte carlo self-consistent field model of physical gels of telechelic polymers", Journal of chemical theory and computation vol. 14, no. 12, 6532-6543 (2018).

${ }^{165}$ Leermakers, F., Bergsma, J., and Van Der Gucht, J., "Hybrid monte carlo selfconsistent field approach to model a thin layer of a polyelectrolyte gel near an adsorbing surface", The Journal of Physical Chemistry A vol. 116, no. 25, 6574-6581 (2012).

${ }^{166}$ Imiya, A. and Eckhardt, U., "The euler characteristics of discrete objects and discrete quasi-objects", Computer Vision and Image Understanding vol. 75, no. 3, 307-318 (1999). 
${ }^{167}$ Allendoerfer, C. B. and Weil, A., "The gauss-bonnet theorem for riemannian polyhedra", Transactions of the American Mathematical Society vol. 53, no. 1, 101-129 (1943).

${ }^{168}$ Harer, J. and Zagier, D., "The euler characteristic of the moduli space of curves", Inventiones mathematicae vol. 85, no. 3, 457-485 (1986).

${ }^{169}$ Sun, D. and Guo, H., "Influence of compositional gradient on the phase behavior of ternary symmetric homopolymer-copolymer blends: a monte carlo study", Polymer vol. 52, no. 25, 5922-5932 (2011).

${ }^{170}$ Lange, N. de, Kleijn, J. M., and Leermakers, F., "Structural and mechanical parameters of lipid bilayer membranes using a lattice refined self-consistent field theory", Physical Chemistry Chemical Physics, https://doi.org/10.1039/ D0CP05597B (2021).

${ }^{171}$ Edelsbrunner, H., Kirkpatrick, D., and Seidel, R., "On the shape of a set of points in the plane", IEEE Transactions on information theory vol. 29, no. 4, 551-559 (1983).

${ }^{172}$ Wong, P.-z., "Scattering by inhomogeneous systems with rough internal surfaces: porous solids and random-field ising systems", Physical Review B vol. 32, no. 11, 7417 (1985).

${ }^{173}$ Bale, H. D. and Schmidt, P. W., "Small-angle x-ray-scattering investigation of submicroscopic porosity with fractal properties", Physical Review Letters vol. 53 , no. 6, 596 (1984).

${ }^{174}$ Sinha, S., Sirota, E., Garoff, S., and HB, S., "X-ray and neutron scattering from rough surfaces", Physical Review B vol. 38, no. 4, 2297 (1988).

${ }^{175}$ Tijum, R. van, Interface and surface roughness of polymer-metal laminates (University Library Groningen, 2006).

${ }^{176}$ Blokhuis, E. M. and Bedeaux, D., "Van der waals theory of curved surfaces", Molecular Physics vol. 80, no. 4, 705-720 (1993).

${ }^{177}$ Vink, R. and Horbach, J., "Critical behaviour and interfacial fluctuations in a phase-separating model colloid-polymer mixture: grand canonical monte carlo simulations", Journal of Physics: Condensed Matter vol. 16, no. 38, S3807 (2004).

${ }^{178}$ Lehle, H., Oettel, M., and Dietrich, S., "Effective forces between colloids at interfaces induced by capillary wavelike fluctuations", EPL (Europhysics Letters) vol. 75 , no. 1,174 (2006).

${ }^{179}$ Schlossman, M. L., "Liquid-liquid interfaces: studied by x-ray and neutron scattering", Current opinion in colloid \& interface science vol. 7, no. 3-4, 235-243 (2002). 
${ }^{180}$ Kolmogorov, A. N., "A refinement of previous hypotheses concerning the local structure of turbulence in a viscous incompressible fluid at high reynolds number", Journal of Fluid Mechanics vol. 13, no. 1, 82-85 (1962).

${ }^{181}$ Pope, S. B., Turbulent flows, 2001.

${ }^{182}$ Meunier, J., "Liquid interfaces: role of the fluctuations and analysis of ellipsometry and reflectivity measurements", Journal de Physique vol. 48, no. 10, 1819-1831 (1987).

${ }^{183}$ Gompper, G. and Kraus, M., "Ginzburg-landau theory of ternary amphiphilic systems. i. gaussian interface fluctuations", Physical Review E vol. 47, no. 6, 4289 (1993).

${ }^{184}$ Höfling, F. and Dietrich, S., "Enhanced wavelength-dependent surface tension of liquid-vapour interfaces", EPL (Europhysics Letters) vol. 109, no. 4, 46002 (2015).

${ }^{185}$ Parry, A., Rascón, C., Willis, G., and Evans, R., "Pair correlation functions and the wavevector-dependent surface tension in a simple density functional treatment of the liquid-vapour interface", Journal of Physics: Condensed Matter vol. 26 , no. 35,355008 (2014).

${ }^{186}$ Fernández, E. M., Chacón, E., Tarazona, P., Parry, A. O., and Rascón, C., "Intrinsic fluid interfaces and nonlocality", Physical review letters vol. 111, no. 9, 096104 (2013).

${ }^{187}$ Tolman, R. C., "The effect of droplet size on surface tension", The journal of chemical physics vol. 17, no. 3, 333-337 (1949).

${ }^{188}$ Blokhuis, E. M. and Kuipers, J., "Thermodynamic expressions for the tolman length", The journal of chemical physics vol. 124, no. 7, 074701 (2006).

${ }^{189}$ Dawson, D. A., "Critical dynamics and fluctuations for a mean-field model of cooperative behavior", Journal of Statistical Physics vol. 31, no. 1, 29-85 (1983).

${ }^{190}$ Shiino, M., "Dynamical behavior of stochastic systems of infinitely many coupled nonlinear oscillators exhibiting phase transitions of mean-field type: $\mathrm{h}$ theorem on asymptotic approach to equilibrium and critical slowing down of order-parameter fluctuations", Physical Review A vol. 36, no. 5, 2393 (1987).

${ }^{191}$ Mukamel, D. and Pytte, E., "Interface fluctuations and the ising model in a random field", Physical Review B vol. 25, no. 7, 4779 (1982).

${ }^{192}$ Parry, A. O. and Boulter, C., "Fluctuation theory for the wavevector expansion of the excess grand potential of a liquid-vapour interface and the theory of interfacial fluctuations", Journal of Physics: Condensed Matter vol. 6, no. 36, 7199 (1994). 
${ }^{193}$ Tarazona, P., Checa, R., and Chacón, E., "Critical analysis of the density functional theory prediction of enhanced capillary waves", Physical review letters vol. 99, no. 19, 196101 (2007).

${ }^{194}$ Parry, A., Rascón, C., and Evans, R., "Liquid-gas asymmetry and the wavevector-dependent surface tension", Physical Review E vol. 91, no. 3, 030401 (2015).

${ }^{195}$ Fradin, C., Braslau, A., Luzet, D., Smilgies, D., Alba, M., Boudet, N., Mecke, K., and Daillant, J., "Reduction in the surface energy of liquid interfaces at short length scales", Nature vol. 403, no. 6772, 871-874 (2000).

${ }^{196}$ Mecke, K. and Dietrich, S., "Effective hamiltonian for liquid-vapor interfaces", Physical Review E vol. 59, no. 6, 6766 (1999).

${ }^{197}$ Mora, S., Daillant, J., Mecke, K., Luzet, D., Braslau, A., Alba, M., and Struth, B., "X-ray synchrotron study of liquid-vapor interfaces at short length scales: effect of long-range forces and bending energies", Physical review letters vol. 90, no. 21, 216101 (2003).

${ }^{198}$ Arleth, L., Marcelja, S., and Zemb, T., "Gaussian random fields with two level-cuts - model for asymmetric microemulsions with nonzero spontaneous curvature?", The Journal of Chemical Physics vol. 115, no. 8, 3923-3936 (2001).

${ }^{199}$ Zemb, T. N., Klossek, M., Lopian, T., Marcus, J., Schöettl, S., Horinek, D., Prevost, S. F., Touraud, D., Diat, O., Marčelja, S., et al., "How to explain microemulsions formed by solvent mixtures without conventional surfactants", Proceedings of the National Academy of Sciences vol. 113, no. 16, 4260-4265 (2016).

${ }^{200}$ Zhou, N., Lodge, T. P., and Bates, F. S., "Influence of conformational asymmetry on the phase behavior of ternary homopolymer/block copolymer blends around the bicontinuous microemulsion channel", The Journal of Physical Chemistry B vol. 110, no. 9, 3979-3989 (2006).

${ }^{201}$ Guerra, C., Somoza, A., and Telo da Gama, M., "Asymmetric water-oilamphiphile mixtures: lamellar phases and droplet microemulsions", The Journal of chemical physics vol. 109, no. 3, 1152-1161 (1998).

${ }^{202}$ Pretti, M. and Buzano, C., "Phase behavior of an asymmetric vector lattice model for oil-water-amphiphile mixtures", The Journal of chemical physics vol. 111, no. 16, 7624-7635 (1999).

${ }^{203}$ Buzano, C. and Pretti, M., "A microemulsion model on sc, bcc and fcc lattices: ground state properties", The Journal of Chemical Physics vol. 113, no. 24, 11364-11371 (2000). 
${ }^{204}$ Hsu, L., Kusner, R., and Sullivan, J., "Minimizing the squared mean curvature integral for surfaces in space forms", Experimental Mathematics vol. 1, no. 3, 191-207 (1992).

${ }^{205}$ Altevogt, P., Evers, O. A., Fraaije, J. G., Maurits, N. M., and Vlimmeren, B. A. van, "The mesodyn project: software for mesoscale chemical engineering", Journal of Molecular Structure: THEOCHEM vol. 463, no. 1-2, 139-143 (1999).

${ }^{206}$ Emmery, D., "Mesodyn simulations of liquid liquid interface", WUR vol. 2020.

${ }^{207}$ Onuki, A., "Nonlinear theory of sound waves near critical points. i: - new dynamic regime -", Progress of Theoretical Physics vol. 66, no. 4, 1230-1249 (1981).

${ }^{208}$ Onuki, A., "Nonlinear theory of sound waves near critical points. ii: - periodic spinodal decomposition in systems with nonconserved order parameter-", Progress of Theoretical Physics vol. 67, no. 3, 768-786 (1982).

${ }^{209}$ Onuki, A., "Nonlinear theory of sound waves near critical points. iii: - first order phase transition under periodic temperature - ", Progress of Theoretical Physics vol. 67 , no. $3,787-803$ (1982).

${ }^{210}$ Lange, N. de, Leermakers, F. A. M., and Klein, M., "Structural and mechanical parameters of lipid bilayer membranes using a lattice refined self-consistent field theory", in preparation. 



\section{Summary (Tamil)}





$$
\text { சுருக்கவுரை }
$$

எண்ணெய்யும் தண்ணீரும் ஓன்றுடன் ஓன்று இணையாது. உதாரணத்திற்கு தண்ணீர் நிரப்பிய கோப்ணையில் சிறிது எண்ணெய் ஊற்றினால் என்ன நடக்(ும் என்று சிந்தனை செய்து பாருங்கள்! இதை நாம் நமது அன்றாட வாழ்வில் பல முறை பார்த்திருப்போம். எண்ணொய் தண்ணீருட் கலக்காமல் பிரிந்து மேலே மிதக்கும்.

உலகின் அணைன்து பொருட்களும் அணுக்களால் ஆனவை. எண்ணெய் மூலக்கூறு|கள் பெரும்பாலும் கார்பன் எனப்படும் கரிப்பபாருள் அணுக்களால் ஆனவை. தண்ணீரின் மூலக்கூறுகள் ஹைட்ரஜன் எனப்படும் ஜல வாயு அணு|க்களால் ஆனவை. இவ்விரு அணுக்கள் இடையே இயங்(கும் விசையை மூலக்கடற்று இடைவிசை என்று கூறுவார்கள். இந்த மூலக்கூற்று இடைவிசை ஈர்ப்பு அல்லது எதிர்ப்பு விசை என மேலும் இரண்டு வகைப்படும். கார்பன் மற்றும் ஹைட்ரஜன் மூலக்ஃறுகள் இடையே எதிர்ப்பு மூலக்ஃ்ற்று இடைவிசை இருப்யதால் எண்ணெயும் தண்ணீரும் இயல்பு நிலையில் பிரிந்தே இருக்கும்.

பல தொழில்துறை சார்ந்த பயன்பாடுகளுக்கு தண்ணீருடன் கலக்கும் திறன் பெற்ற எண்ணெய் தேலைவப்படிகிறது. உதாரணத்திற்கு நிலத்தடி கச்சா எண்ணெய்ணய வெளியில் எடுக்க பூமியில் குழாய்களில் ஏற்படுத்தி அக்குழாய்கள் மூலம் தண்ணீர் செலுத்தப்படும். அவ்வாறு செலுத்தும் தண்ணீர் கச்சா எண்ணேய்பயயாடு கலந்து வெளியில் கலவை நிலையில் வரும். இந்த கலவையை சுத்தீகரித்து வாகன எரிபெருள் போன்ற பொருட்கள் தயாரிக்கப்படும். இதில் ஓரே ஓரு சிக்கல் மட்டுமே. நாம் முண்னே ஈறறியவாறு தண்ணீருடன் இயல்பாக எண்ணெய் கலவை நிலைக்கு செல்லாது. தண்ணீர்- எண்ணெய் கலவை உருவாக்க உந்துகோள் தேவை.

துணிகளில் இருக்கும் எண்ணெய் கறைகளை அகற்ற நாம் சலவைத்தாள் உபயோகிக்கிறோம். சலவைத்தாள்களும் மூலக்கூறுகளால் ஆனவையே. அவற்றிற்கு ஓரு சிற்புபு தன்மை உண்டு. अலவைத்தூளின் மூலக்கூறுகள் கார்பன் மற்றும் ஹைட்ரஜன் அணுக்களால் இணைத்து செய்யப்பட்டவை. அவ்வாறு இருக்கும் மூலக்கூறுகளை தண்ணீர்-எண்ணெய் பிரிந்து இருக்கும் கோப்பையில் போட்டால் அவை தண்ணீருக்கும் எண்ணெய்க்கும் இடையே சென்று நிற்கும். இதனால் தண்ணீருக்கும் எண்ணெய்க்கும் இடையே இருக்கும் எதிர்புபு விசை சற்றற குறையும். இவ்வாறு ஓரு மூன்றாம் உந்துகோள் மூலம் தண்ணீர்-எண்ணெய் கலவையை உருவாக்கலாம். இப்படி உருவான கலவையை நாம் நுண்குழம்புகள் என்று அழைக்கிழோம்.

இத்தகைய கலவைகளின் இயற்பியமை அடிய்பை சமன்பாடுகளால் விவரிக்க முடியும். இருப்பிறும் இதுபோன்ற சமண்பாடுகளை கணினிகளைப் பயன்படுத்தி மட்டுமே தீர்க்க முடியும். இந்த ஆய்வறிக்கையில், இதுபோன்ற சமன்பாடுகளை தீர்க்க கணினிகளைப் பயன்படுத்துகிறோம். அவ்வாறு செய்ய, பல கணக்கீட்டு நுட்பங்கள் உருவாக்கப்பட்டனர. உருவாக்கப்பட்ட நுட்பங்களின் தொகுப்பு மற்று|ம் டெற்ப்பட்ட ஈ டுதல் நுண்ணறிவுகளின் விரிவுரையே இந்த ஆய்வுக்கட்டுனை. 



\section{List of publications}

1. R. Varadharajan, F. A. M. Leermakers, Imposing height undulations on the liquid-liquid interface in a Monte Carlo Self-consistent field approach, submitted, (Chapter 5).

2. R. Varadharajan, F. A. M. Leermakers, The physics of microemulsions extracted from modeling balanced tensionless surfactant-loaded liquid-liquid interfaces, The Journal of Chemical Physics 152 (9), 094902, 2020 (Chapter 4).

3. R. Varadharajan, F. A. M. Leermakers, Elastic properties of symmetric liquidliquid interfaces, Physical Review E 100 (6), 062801, 2019, (Chapter 2).

4. R. Varadharajan, F. A. M. Leermakers, Sign switch of Gaussian bending modulus for microemulsions: A self-consistent field analysis exploring scale invariant curvature energies, Physical Review Letters 120 (2), 028003, 2018, (Chapter 3). 



\section{Acknowledgements}

It was a leap of hope. I had to choose between becoming an Engineer in Rolls-Royce and becoming a Ph.D. Researcher in Wageningen University. While the former offered me a safe route in my 'then area of expertise' (Aerodynamics), the latter was full of unknowns. Before accepting the position, I had never boarded an airplane (I sure knew how to build one theoretically), I had never crossed the borders of India, I had never been in a situation where I can only communicate in English, I never had to cook my food, I never had to build my bed. It was a scary journey. I took this leap of hope with a desire to learn a new field. Frans, you made this journey not just less-scary but an enjoyable one. I am thankful for the knowledge I received from our discussions, be it in a car while traveling to Prague or in a coffee break, or during several of our formal and informal meetings. You were always there for help, even at times when you were forced to rest. All the words I know in any language can't do justice to express how thankful I am, to have had you as my supervisor.

Thanks, Jasper. Without your inputs and help, Chapter 4 of this thesis would have been a dream. Your support amidst your busy schedule helped me see the light at end of the tunnel. I cherish the memories during the Han-sur-lesse, lectures by yourself and others helped me gain vast knowledge in a short time. Thanks, Renko, for being there for help and support. Your inputs and constructive feedback on the Introduction of the thesis greatly improved its readability.

Thanks, Valliappan. I hated structural mechanics until I met you. Your lectures on structural mechanics played an important part in my path towards obtaining a place in the Indian Institute of Science. Our relationship slowly changed from a teacher-student to senior-junior to colleagues to friends. I would not have even known the existence of the CSER program, if not for you. You helped me refine my application for the program. You were there during the initial years of my Ph.D. as a good friend. I will always cherish the memories of spending sleepless nights discussing capitalism and walking in the streets of Paris while discussing Democracy and Psychology. Thanks, Jahnavi for joining us in some of those discussions and 
not finding us boring. The trip to Chamrousse was awesome. It happened because of you and helped me get my long-waited vacation.

Niek, you joined a few months after I started in the PCC. After which you had been there at every point of my Ph.D. Be it at winter school in Belgium or a conference in Rotterdam or behind my office desk, playing AFK Arena throughout your Ph.D. You introduced me to several board games. You made me taste alcohol for the first time. You got me temporarily addicted to AFK Arena. You made the Netherlands feel like home. Not thanking or acknowledging you yet, for this is not the end. Thanks, Huy Duc Vu Nguyen, for allowing me to lose many of your ranked chess games. It was nice to have you in the office and you always had accompanied me when I procrastinate watching badminton tournaments (even though you only watched it because you were obsessed with Nozomi Okuhara and Michelle Li!). If not for you guys, I would have completed my Ph.D. within a year. But the fun is worth the additional three years! Thanks, Sven. You were the most dedicated researcher in our office. Indirectly, you motivated me to keep my procrastination under check.

Thanks, Mara. I landed in the Netherlands with no worries as everything was already planned and set by you. From the moment, where you helped me find a room to rent, till the moment where you helped me get a defense date (two times), you had always taken care of planning and organization (the one thing which both I and Frans can never do as good as you even in hundred years). If not for your management skills we would have been lost. Thanks, Diane, for always catching up and saying a "Hi!". Thanks, Remco for your excellent lectures on Light scattering.

Thanks to all the researchers and members of PCC.

Thanks Nick Fransman, you always made me feel special when I am on the court! Thanks Loeck Scholten, Patteela Prathumars, Maarten Immerzeel, William Setiawan, Lars Onderwater, Sven Verwoorts, Sjoerd van Capelleveen and other badminton sparring mates, for creating a healthy environment that helped me relax.

Thanks, Sneha, Kavya, and Karthick, for making the final days of my Ph.D. less stressful!

Thanks, Saravana Kumar and Punithan, for spoiling me with liquor, idlies, video games, random weekend trips, etc.! Thanks, Abinesh, Anish, Gopalsamy, Vibina, Samhitha and others who constantly kept in touch even when I always failed to do the same! 


\section{About the Author}

Ramanathan Varadharajan was born on the 25th of March, 1993, in Tamil Nadu, India. He went to high school at the Krishnasamy matriculation and graduated his studies in 2010 with a focus on Mathematics and Biology.

Later that year he started his bachelor studies in Aeronautical Engineering. As a part of the studies, he performed an internship at Vikram Sarabhai Space Center, ISRO, Kerala, India on numerical grid generation. During the year 2014, he submitted his bachelor's thesis on "Nozzle contour design using the method of characteristics." In the year 2014, he graduated and pursued a master's degree in Aerospace Engineering at the Indian Institute of Science, Bangalore. During this period, as a part of his studies, he completed his thesis on "Large-Eddy simulations on free jets and jets impinging on cuboidal cavities," in the Laboratory of Turbulence Computations, in 2016.

Later in 2016, he started to work as a Ph.D. candidate at Wageningen University in the Physical Chemistry and Soft Matter group. This thesis, entitled "A oneparameter model for microemulsions," is the result of that Ph.D. project. 



\section{Overview of completed training}

\section{Discipline specific activities}

Activity
CSER Future energy Conference
MolSim winterschool
Han-sur-Lesse Winterschool
Biomolecular Macrosystems Conference
Han-sur-Lesse Winterschool
IACIS
ISP (PRE conference PHD course + con-
ference)
RPK-B Polymer Physics

\section{Activity}

MolSim winterschool

Han-sur-Lesse Winterschool

Biomolecular Macrosystems Conference

Han-sur-Lesse Winterschool
IACIS
ISP (PRE conference PHD course + con- RPK-B Polymer Physics

\begin{tabular}{l|l} 
Organizing institute & Year \\
Royal Dutch Shell & 2016 \\
University of Amsterdam & 2017 \\
PCC, Wageningen Uni- & 2017 \\
versity & \\
IMS, Russia & 2017 \\
PCC, Wageningen Uni- & 2018 \\
versity & \\
Wageningen University, & 2018 \\
TU Delft & \\
VLAG & 2018 \\
PTN & 2018
\end{tabular}

\section{General courses}

\section{Course}

Taking charge of your Ph.D.

Saving time by writing better code

Scientific writing

Art of presenting science

Career planning course

Brain training

\section{Organizing institute}

FOM

FOM

WGS

FOM

FOM

WGS
Year

2017

2017

2018

2018

2020
2019 


\section{Optionals}

Activity

Preparation of research proposal

Research methods Soft Matter (MSc course)

Ph.D. study tour (Oce, Fujifilm)

Group meetings and Simulation meeting
Organizing institute PCC

PCC

$\mathrm{PCC}$

PCC
Year

2016

2018

2017, 2018

2016 - 2020 

This work is part of an Industrial Partnership Programme, "Shell/NWO

Computational Sciences for Energy Research (CSER-16)," of the Foundation for Fundamental Research on Matter (FOM), which is part of the Netherlands Organisation for Scientific Research (NWO) (Project No. 15CSER26).

Printed by Digiforce-Proefschriftmaken.nl in 125 copies 